\title{
Level-raising and symmetric power functoriality, III
}

\author{
Laurent Clozel and Jack A. Thorne*
}

December 10, 2015

\section{Contents}

1 Introduction 1

1.1 Notation . . . . . . . . . . . . . . . . . . . . . . . . 3

2 Admissible representations of a ramified $p$-adic unitary group 4

2.1 A unitary group Hecke algebra . . . . . . . . . . . . . . . . . . . . . . . 4

2.2 Functoriality and base change for the group $U_{n} \ldots \ldots \ldots \ldots$

2.3 Integral structures in semi-stable Hecke modules . . . . . . . . . . . . . . . . . . . . 12

3 Unitary groups and transfer $\quad 18$

3.1 Unitary groups . . . . . . . . . . . . . . . . . . . . . . . . 19

3.2 Local L-packets . . . . . . . . . . . . . . . . . . . . . . . . . . . . . . . . . 20

3.3 Global L-packets . . . . . . . . . . . . . . . . . . . . . . . . . . . . 21

3.4 Transfer factors . . . . . . . . . . . . . . . . . . . . . . . . . . . . . 23

3.5 Proof of Theorem $2.3 \ldots \ldots \ldots \ldots \ldots \ldots \ldots \ldots$

3.6 Compact transfer: proofs . . . . . . . . . . . . . . . . . . . . . . . 28

4 Level-raising and algebraic modular forms 3

4.1 Set-up . . . . . . . . . . . . . . . . . . . . . . . . 33

4.2 Raising the level . . . . . . . . . . . . . . . . . . . . . . . . . 35

5 Level-raising and Galois theory $\quad 37$

5.1 A level-raising theorem . . . . . . . . . . . . . . . . . . . . . 37

5.2 An automorphy lifting theorem . . . . . . . . . . . . . . . . . . . . 44

$6 \quad$ Symmetric power functoriality $\quad 44$

7 Complements: the mixed-parity case $\quad 46$

\section{Introduction}

The simplest case of Langlands' functoriality principle asserts the existence of the symmetric powers Sym $^{n} \pi$ of a cuspidal representation $\pi$ of $\mathrm{GL}_{2}\left(\mathbb{A}_{F}\right)$, where $F$ is a number field. After the publication of Langlands' conjecture [Lan70], Gelbart and Jacquet [GJ78] proved the existence of $\mathrm{Sym}^{2} \pi$. After this, progress was

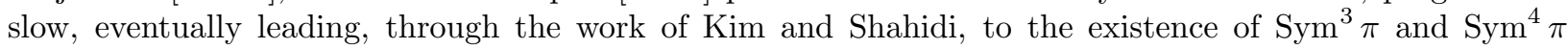
[KS02, Kim03].

* Laurent Clozel is a member of Institut Universitaire de France. During the period this research was conducted, Jack Thorne served as a Clay Research Fellow. 
In this series of papers [CT14, CT15], we revisit this problem using recent progress in the deformation theory of modular Galois representations. As a consequence, our methods apply only to $\pi$ associated to classical modular forms on a totally real number field (in fact, forms of weight at least 2 in each variable, and constant parity). (In this introduction only, we say " $\pi$ is classical" for short. In fact, the assumption on parity can be removed; see $\S 7$ below.) We assume that $\pi$ is not of CM type, i.e. not obtained by automorphic induction from an algebraic Hecke character of a CM extension of $F$. We will say that the $n^{\text {th }}$ symmetric power $\operatorname{Sym}^{n} \pi$ exists if there is a regular algebraic cuspidal automorphic representation $\Pi$ of $\mathrm{GL}_{n+1}\left(\mathbb{A}_{F}\right)$ such that, for any prime $l$,

$$
\operatorname{Sym}^{n} r_{l}(\pi) \cong r_{l}(\Pi)
$$

where $r_{l}(\pi): G_{F}=\operatorname{Gal}(\bar{F} / F) \rightarrow \mathrm{GL}_{2}\left(\overline{\mathbb{Q}}_{l}\right)$ is the $l$-adic representation associated to $\pi$ (for some embedding of its field of coefficients into $\left.\overline{\mathbb{Q}}_{l}\right)$ and $r_{l}(\Pi)$ is similarly defined. Note that this is independent of the apparent choices, by well-known properties of the set of such cuspidal representations under conjugation of the coefficients.

Theorem 1.1 (Theorem 6.1). $\quad$ 1. Assume that $F$ is linearly disjoint from $\mathbb{Q}\left(\zeta_{5}\right)$. Then $\operatorname{Sym}^{6} \pi$ exists.

2. Assume that $F$ is linearly disjoint from $\mathbb{Q}\left(\zeta_{7}\right)$. Then $\mathrm{Sym}^{8} \pi$ exists.

Under similar restrictions on $F$ and $\pi, \operatorname{Sym}^{5} \pi$ and $\operatorname{Sym}^{7} \pi$ were constructed in [CT15]. As a consequence, one now knows:

Corollary 1.2. Assume $F$ linearly disjoint from $\mathbb{Q}\left(\zeta_{35}\right)$, and $\pi$ classical. Then $\mathrm{Sym}^{n} \pi$ exists for $n \leq 8$.

We recall that the "potential" existence of $\Pi$, i.e. the existence of some unspecified Galois base change of $\Pi$, was obtained by Barnet-Lamb, Gee, and Geraghty [BLGG11], following earlier work of Clozel, Harris, Taylor, and Shepherd-Barron [Tay08, Har09, CHT08, HSBT10]. Therefore the new content of our work is the existence of $\Pi$ itself, on its proper ground field. In particular this implies:

Corollary 1.3 (Assumptions of Corollary 1.2). The L-function $L\left(s, \operatorname{Sym}^{n} \pi\right)$ is holomorphic in the whole plane, with the expected functional equation.

We now give some indications on the proof, concentrating for definiteness on the more difficult case, $n=8$. Fix $l=7$, and consider the reduction $\bmod l, \bar{r}$, of $r_{l}(\pi)$. The representation theory of $\mathrm{GL}_{2}\left(\overline{\mathbb{F}}_{7}\right)$ implies, up to semi-simplification, an isomorphism

$$
\operatorname{Sym}^{8} \bar{r} \sim\left({ }^{\varphi} \bar{r} \otimes \bar{r}\right) \oplus \bar{\chi}^{2} \operatorname{Sym}^{4} \bar{r} .
$$

Here $\chi$ is the determinant of $r$, and $\varphi$ is the Frobenius endomorphism acting on the coefficients. Since, as we saw, ${ }^{\varphi} \bar{r}$ is automorphic, both summands of (1.1) are; they are irreducible if the image of $\bar{r}$ is sufficiently large.

Keeping to the real field $F$, we see that $\operatorname{Sym}^{8} \bar{r}$ is of the type considered by Arthur in [Art], i.e. the sum of two essentially orthogonal representations of degrees 4, 5. After some arguments involving, in particular, known results about change of weight, we see that this semi-simple residual representation is associated to an automorphic, cohomological representation $\Sigma$ of the split group $\mathrm{Sp}_{8}$ over $F$ occurring in the discrete spectrum, an "endoscopic" representation. Our arguments could probably be given in this context, but we would need to transfer $\Sigma$ to a form of $\mathrm{Sp}_{8}$ compact at the real primes. This is delicate, and is not completed in [Art, Ch. 9]. We take a different route. We choose a suitable CM extension $E$ of $F$; after base change to $E$ we are given two "regular algebraic, essentially conjugate self-dual, cuspidal" representations $\pi_{4}, \pi_{5}$ of $\mathrm{GL}_{4}\left(\mathbb{A}_{E}\right)$ and $\mathrm{GL}_{5}\left(\mathbb{A}_{E}\right)$. The representation (1.1) becomes a "Schur representation" of unitary type $\bar{R}: G_{E} \rightarrow \mathrm{GL}_{9}\left(\overline{\mathbb{F}}_{7}\right)$ in the sense of [CHT08], [Tho15]. One of us (J.T.) has recently proved that deformation theory can be applied in this context, provided that one knows the existence of an initial, automorphic (cuspidal) lifting $R: G_{E} \rightarrow \mathrm{GL}_{9}\left(\overline{\mathbb{Z}}_{7}\right)$ corresponding to a representation of $\mathrm{GL}_{9}\left(\mathbb{A}_{E}\right)$ that is Steinberg at some finite prime. The problem of automorphy of the symmetric power $\operatorname{Sym}^{8} r_{l}(\pi)$ is thus reduced to the problem of the existence of this Steinberg lifting. It is this problem of "level-raising" that occupies us for most of this paper. 
This level-raising is done by extending arguments introduced in [CT15]. In $§ 2$, we introduce some representations of the odd-dimensional unitary group associated to a ramified quadratic extension of $p$-adic fields, and study their integral structures. The interplay between these integral structures and the endoscopic decomposition of global spaces of automorphic forms plays a key role in the main level-raising argument. These representations are Iwahori-spherical and are constructed from a certain Langlands parameter using the work of Kazhdan and Lusztig [KL87].

In $\S 3$ we construct our initial "endoscopic" representation (with residual representation isomorphic to $\bar{R}$, as described above) as an automorphic representation of a definite unitary group $G$. We also study the local theory and identify the representations constructed in $\S 2$ as being members of a common L-packet by verifying directly the necessary trace identities (see $\S 3.5)$.

We should justify the necessity (in our approach) of the complicated interplay of $\S 2$ and $\S 3$. The $L$-packets described in Theorem 3.2 are perfectly specified by Moeglin and Mok, by two identities of (twisted) traces. However it is crucial for us to understand the Jacquet modules of their elements. The results of Mok and Moeglin are not sufficient for this purpose.

In $\S 4$, we carry out the automorphic part of the level-raising argument, using algebraic modular forms on $G$. In contrast to the situation of [CT15], although this argument does produce a new automorphic representation that is 'more ramified' than the initial one, we can no longer guarantee that this representation is Steinberg locally (the possibilities are enumerated in Proposition 4.1). Moreover, the level-raising argument succeeds only for $l=5,7$, whereas the identity corresponding to (1.1), for $\mathrm{Sym}^{l+1}$, holds for an arbitrary prime $l$. The reason for this failure is given after Lemma 4.4. This is also in contrast to [CT15], where we were able to establish a result for any prime $l>3$.

We are therefore led in $\S 5$ to prove another level-raising result, which uses deformation theory to move from the representation constructed in $\S 4$ to one which is indeed Steinberg locally. We note that an argument of Taylor (see [Tay08], [Gee11]) allows one to construct automorphic liftings of an irreducible residual Galois representation with essentially any prescribed local behaviour (for example, with Steinberg type ramification). The key difficulty for us is that we must work with a reducible residual Galois representation (as necessitated e.g. by the equation (1.1)). However, it turns out that the ideas pertaining to the deformation of reducible residual representations introduced in [Tho15] are strong enough to allow one to produce a Steinberg lifting, the key point being that the initial automorphic lift (provided by the work done in $\S 4$ ) does have a place where the local representation is incompatible with the global Galois representation being globally reducible (see especially Lemma 5.3).

Having constructed this automorphic lifting of $\bar{R}$, Steinberg at some finite place, we can apply the automorphy lifting theorem of [Tho15], much as in our previous paper, to obtain the automorphy of the symmetric power $\operatorname{Sym}^{8} r_{l}(\pi)$, and we carry this out in $\S 6$. In fact, we phrase things slightly differently by stating a new automorphy lifting theorem (Theorem 5.7) and applying it to the datum constructed in $\S 4$. We hope that this automorphy lifting theorem will have applications elsewhere. Finally, in $\S 7$ we show how to reduce the existence of symmetric power liftings of representations of $\mathrm{GL}_{2}\left(\mathbb{A}_{F}\right)$ which are essentially square-integrable at infinity to the corresponding problem for those which are regular algebraic up to twist (and which is solved in some cases in $\S 6$ ).

We note that as in our earlier paper [CT15], our proofs rely on the work of Mok [Mok15], extending Arthur's results to unitary groups; and that in turn Mok's results are conditional on the stabilisation of the twisted trace formula. This has now been announced in preprint form by Møglin and Waldspurger [MW]. Finally, we again thank Møglin, here for providing a proof of Proposition 3.7. We also thank all the referees for corrections and criticisms which, we hope, have substantially improved the exposition.

\subsection{Notation}

We refer to $[\mathrm{CT} 14, \S 2]$ for basic notations relating to automorphic representations, including in particular the local Langlands correspondences $\operatorname{rec}_{F_{v}}$ and $\operatorname{rec}_{F_{v}}^{T}$ for $\mathrm{GL}_{n}$; the definition of RACSDC, RAESDC, RACSD automorphic representations of $\mathrm{GL}_{n}\left(\mathbb{A}_{F}\right)$; the notion of an $\iota$-ordinary automorphic representation; the weight $\boldsymbol{\lambda}$ and infinity type $\boldsymbol{a}$ of a regular algebraic, cuspidal automorphic representation; and the existence of Galois representations $r_{\iota}(\pi)$, and what it means for a Galois representation $\rho$ to be automorphic. 
If $F$ is a field, then we write $G_{F}$ for its absolute Galois group. If $F$ is a number field and $v$ is a finite place of $v$, then we will write $G_{F_{v}}$ for a choice of decomposition group at the place $v$; it is endowed with a homomorphism $G_{F_{v}} \rightarrow G_{F}$, determined up to $G_{F}$-conjugacy. We write $q_{v}=\# k(v)$ for the size of the residue field at $v$. If $F$ is a number field and $S$ is a finite set of finite places of $F$, we write $G_{F, S}$ for the Galois group of the maximal extension of $F$ unramified outside $S$. If $v \notin S$ is a finite place of $F$, then we write $\operatorname{Frob}_{v} \in G_{F, S}$ for a (geometric) Frobenius element, uniquely determined up to $G_{F, S}$-conjugacy. If $l$ is a prime and $\rho: G_{F} \rightarrow \mathrm{GL}_{n}\left(\overline{\mathbb{Q}}_{l}\right)$ is a continuous representation, then after conjugation we can assume that $\rho$ takes values in $\mathrm{GL}_{n}\left(\overline{\mathbb{Z}}_{l}\right)$. The semi-simplification of the composite $G_{F} \rightarrow \mathrm{GL}_{n}\left(\overline{\mathbb{Z}}_{l}\right) \rightarrow \mathrm{GL}_{n}\left(\overline{\mathbb{F}}_{l}\right)$ is independent of this choice, up to isomorphism, and will be denoted by $\bar{\rho}$.

If $G$ is a reductive group over a non-Archimedean local field $F, P$ is a parabolic subgroup of $G$, and $\sigma$ is an admissible representation of $P$, then we write $\mathrm{n}-\operatorname{Ind}_{P}^{G} \sigma$ for the normalized (unitary) induction of $\sigma$, an admissible representation of $G$. If $N$ is the unipotent radical of $P$ and $\pi$ is an admissible representation of $G$, then we write $\pi_{N}^{\text {norm }}$ for the normalized Jacquet module of $\pi$. We will write $\mathrm{St}_{G}$ for the Steinberg representation of $G$. If $G=\mathrm{GL}_{n}(F)$ then we will write $\mathrm{St}_{n, F}$ for the Steinberg representation.

\section{Admissible representations of a ramified $p$-adic unitary group}

In this section, which is similar in nature to $[\mathrm{CT} 15, \S 2]$, we study the representations of ramified $p$-adic unitary groups which play a key role in the 'automorphic part' of the level-raising arguments in $\S 4$. As in [CT15], these arguments will rely on fine properties of the local L-packet containing these representations. The reason for considering ramified groups is that only for those can we obtain the crucial Proposition 2.9; for unramified groups this multiplicity-one property fails.

The representations we consider are all Iwahori-spherical. We therefore begin by studying a certain Hecke algebra for the ramified unitary group $U_{n}$. We then construct certain representations of this group (see Theorem 2.5) using the theory of Kazhdan-Lusztig [KL87], and study their integral structures (see Proposition 2.6) using the theory of Reeder [Ree00]. Ultimately, the representations we construct will be shown to be the Iwahori-spherical members of a certain L-packet; we state this result as Theorem 2.3, which will however be proved only in $\S 3.5$.

\subsection{A unitary group Hecke algebra}

Let $p$ be an odd prime, $F$ a finite extension of $\mathbb{Q}_{p}$, and $E$ a ramified quadratic extension of $F$. If $t \in E$, we will write $t^{c}$ or $\bar{t}$ to denote the image of $t$ under the non-trivial element of $\operatorname{Gal}(E / F)$. We write $k_{F}$ for the residue field of $F$, and $q=\# k_{F}$. Fix a uniformizer $\varpi$ of $E$ such that $\varpi^{2} \in F$, and let $v: E^{\times} \rightarrow \mathbb{Z}$ denote the valuation with $v(\varpi)=\frac{1}{2}$. Let $n=2 k+1 \geq 1$ be an odd integer. Let $I=\{ \pm 1, \ldots, \pm k\}$, and identify $E^{n}$ with the set of vectors $\left(x_{1}, \ldots, x_{k}, x_{0}, x_{-k}, \ldots, x_{-1}\right)$ with entries $x_{i} \in E$. There is, up to isomorphism, a unique outer form of $\mathrm{GL}_{n}$ over $F$ which is split by $E$. One choice is as follows. Define the $n \times n$ matrix

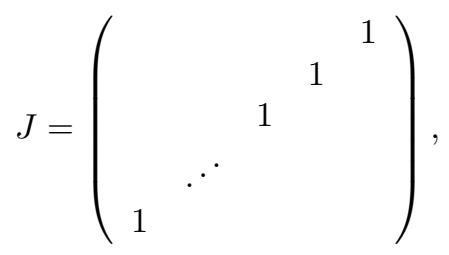

and let $\mathcal{U}_{n}$ denote the smooth affine group scheme over $\mathcal{O}_{F}$ whose $R$-points are

$$
\mathcal{U}_{n}(R)=\left\{g \in \mathrm{GL}_{n}\left(R \otimes_{\mathcal{O}_{F}} \mathcal{O}_{E}\right){ }^{t} g J g^{c}=J\right\} .
$$

Let $U_{n}$ denote the $F$-fiber of $\mathcal{U}_{n}$, a reductive group over $F$. Its derived group is $S U_{n}$, the subgroup of matrices with determinant 1 , which is simply connected. We will begin by describing the building $\mathscr{B}\left(U_{n}, F\right)$ of the group $U_{n}$, following [Tit79]. A maximal $F$-split torus of $U_{n}$ is the subgroup $S \subset U_{n}$ given by the matrices

$$
\operatorname{diag}\left(t_{1}, \ldots, t_{k}, 1, t_{-k}, \ldots, t_{-1}\right), t_{i} \in F^{\times}, t_{i} t_{-i}=1 .
$$


This is also a maximal $F$-split torus of $S U_{n}$. We define cocharacters $e_{1}, \ldots, e_{k}: \mathbb{G}_{m} \rightarrow S$ by the formulae

$$
e_{i}(t)=\left(1, \ldots, t, \ldots, t^{-1}, \ldots, 1\right),
$$

where $t$ sits in the $i$-entry and $t^{-1}$ in the $(-i)$-entry; these cocharacters form a basis of the free $\mathbb{Z}$-module $X_{*}(S)$. We write $a_{1}, \ldots, a_{k}$ for the dual basis of $X^{*}(S)$. If $1 \leq i \leq k$, then we also define $a_{-i}=-a_{i} \in X^{*}(S)$.

We write $\Phi=\Phi\left(U_{n}, S\right)$ for the root system of $S$. If we define $a_{i j}=a_{i}+a_{j} \in X^{*}(S)$ for $i, j \in I$, then we have

$$
\Phi=\left\{a_{i j} \mid i, j \in I, i \neq \pm j\right\} \cup\left\{a_{i}, 2 a_{i} \mid i \in I\right\} .
$$

This is a non-reduced root system of type $\mathrm{BC}_{k}$. We write $W_{0}$ for its Weyl group. Viewing $\mathrm{BC}_{k}$ as the union of its subsystems $\mathrm{B}_{k}$ and $\mathrm{C}_{k}, W_{0} \cong\{ \pm 1\}^{k} \rtimes \mathfrak{S}_{k}$ may be identified with the Weyl group of either one of these subsystems. There is a natural action of $W_{0}$ on the set $I \cup\{0\}$. Let $Z=Z_{U_{n}}(S)$. This is a maximal torus of $U_{n}$, consisting of the matrices

$$
\operatorname{diag}\left(t_{1}, \ldots, t_{k}, t_{0}, t_{-k}, \ldots, t_{-1}\right), t_{i} \in E^{\times}, t_{i} t_{-i}^{c}=1 \text { for each } i=0, \ldots, k .
$$

We let $Z_{c} \subset Z$ denote the maximal compact subgroup, and set $\Lambda=Z / Z_{c} \cong \mathbb{Z}^{m}$, a basis being given by the elements

$$
\epsilon_{i}=\operatorname{diag}(1, \ldots, \varpi, \ldots,-1 / \varpi, \ldots, 1), 1 \leq i \leq k,
$$

where $\varpi$ occupies the $i$-entry. The group $X^{*}(Z)=\operatorname{Hom}_{F}\left(Z, \mathbb{G}_{m}\right)$ embeds as a finite index subgroup of $X^{*}(S)$ (via restriction), and there is a natural embedding $\nu: \Lambda \hookrightarrow V=X_{*}(S) \otimes_{\mathbb{Z}} \mathbb{R}$ which is characterized by the formula $\chi(\nu(z))=-v(\chi(z))\left(z \in Z, \chi \in X^{*}(Z)\right)$. We let $N=N_{U_{n}}(Z)$, and $W=N / Z_{c}$ the extended affine Weyl group of $U_{n}$ with respect to $S$; it is an extension of $\Lambda$ by $N / Z \cong W_{0}$.

The standard apartment $A\left(U_{n}, S, F\right)$ consists of the following data (see [Tit79, 1.2]):

- An affine space $A=A\left(U_{n}, S, F\right)$ under the real vector space $V=X_{*}(S) \otimes_{\mathbb{Z}} \mathbb{R}$, equipped with an action of the group $W=N / Z_{c}$ which extends the translation action of $\Lambda$ via $\nu$.

- A collection $\Phi_{\mathrm{af}}=\Phi_{\mathrm{af}}\left(U_{n}, S, F\right)$ of affine functions $A \rightarrow \mathbb{R}$ and a mapping $\alpha \mapsto X_{\alpha}$ which assigns to each $\alpha \in \Phi_{\text {af }}$ a subgroup $X_{\alpha}$ of $U_{n}$. The elements of $\Phi_{\text {af }}$ are called the affine roots of $U_{n}$ with respect to $S$.

These data satisfy some additional conditions that we do not describe here. Having defined the standard apartment, the building $\mathscr{B}=\mathscr{B}\left(U_{n}, F\right)$ is the unique $U_{n}$-set up to isomorphism satisfying the following conditions (see [Tit79, 2.1]):

- $\mathscr{B}$ contains $A$, and is the union of its $U_{n}$-translates.

- The group $N \subset U_{n}$ stabilizes $A$ and its action coincides the given one.

- For each affine root $\alpha \in \Phi_{\mathrm{af}}$, the group $X_{\alpha}$ fixes the half-apartment $A_{\alpha}=\alpha^{-1}([0, \infty))$ pointwise.

If $\Omega \subset \mathscr{B}$ is a subset, then we write $U_{n}^{\Omega}$ for the subgroup fixing $\Omega$ pointwise. If $\Omega \subset A$, then $U_{n}^{\Omega}$ can be described explicitly in terms of the affine root system (see [Tit79, 3.1]).

We now describe the standard apartment for the group $U_{n}$. The case of the special unitary group $S U_{n} \subset U_{n}$ is treated in detail in [Tit79, 1.15], and is very similar. We can identify $A=V \cong \mathbb{R}^{n}$, with basis $e_{1}, \ldots, e_{k}$. The group $N$ consists of matrices $n\left(\sigma, a_{1}, \ldots, a_{-1}\right)=\left(g_{r s}\right), g_{r s}=\delta_{r \sigma(s)} a_{s}$ ( $\delta$ is the Kronecker delta), for all $\sigma \in W_{0}$ and $a_{i} \in E$ such that $a_{i} a_{-i}^{c}=1$ for all $i=0, \ldots, k$. The action of $N$ on $V$ is given by the formula

$$
n\left(\sigma, a_{1}, \ldots, a_{-1}\right)\left(\sum_{i=1}^{k} x_{i} e_{i}\right)=\sum_{i=1}^{k} y_{i} e_{i},
$$

where $y_{\sigma(i)}=x_{i}+v\left(a_{-i}\right)$ and we define $y_{-i}=-y_{i}$. The affine roots are the functions $(i, j \in I)$

$$
\left\{a_{i j}+m \mid i \neq \pm j, m \in \frac{1}{2} \mathbb{Z}\right\} \cup\left\{a_{i}+m \mid m \in \frac{1}{2} \mathbb{Z}\right\} \cup\left\{2 a_{i}+\frac{1}{2}+m \mid m \in \mathbb{Z}\right\} .
$$


We recall that the chambers of $A$ are by definition the connected components of the complement of the vanishing hyperplanes of the affine roots $\alpha \in \Phi_{\mathrm{af}}$. A chamber is given in this case by the series of inequalities

$$
C: 0<a_{k}<a_{k-1}<\cdots<a_{1}<\frac{1}{4} .
$$

The corresponding root basis is $\left\{\alpha_{0}, \ldots, \alpha_{k}\right\}=\left\{2 a_{-1}+\frac{1}{2}, a_{1,-2}, a_{2,-3}, \ldots, a_{k-1,-k}, a_{k}\right\}$. Since every element of the quotient $W=N / Z_{c}$ can be represented by an element of the group $N \cap S U_{n}$, and $S U_{n}$ is simply connected, we see that $W$ is generated by the corresponding set $\left\{s_{0}, \ldots, s_{k}\right\}$ of simple reflections [Tit79, 1.13]. The affine reflection $s_{0} \in W$ corresponding to the simple affine root $\alpha_{0}=2 a_{-1}+\frac{1}{2}$ is, by definition, the unique reflection in $A \cong V$ with vector part $s_{a_{-1}}$ and fixed hyperplane $2 a_{-1}+\frac{1}{2}=0$. For later use, we note the expression (for $\sum_{i} x_{i} e_{i} \in V$ ):

$$
s_{0}\left(\sum_{i=1}^{k} x_{i} e_{i}\right)=\sum_{i=2}^{k} x_{i} e_{i}+\left(\frac{1}{2}-x_{1}\right) e_{1} .
$$

The point 0 of $A$ is a special vertex [Tit79, 1.9], and there is an induced semi-direct product decomposition $W=W_{0} \ltimes \Lambda$, where we identify $W_{0}=\left\langle s_{1}, \ldots, s_{k}\right\rangle \subset W$. The corresponding group scheme of this special vertex ([Tit79, 3.4]) is canonically identified with $\mathcal{U}_{n}$. In particular, the group $K=\mathcal{U}_{n}\left(\mathcal{O}_{F}\right)$ is a special subgroup of $U_{n}$. It follows from [Tit79, 3.1] that the group $\mathfrak{B}=U_{n}^{C}$ is the pre-image in $K$ under the canonical reduction map $\mathcal{U}_{n}\left(\mathcal{O}_{F}\right) \rightarrow \mathcal{U}_{n}\left(k_{F}\right) \rightarrow \mathrm{O}(J)\left(k_{F}\right)$ of the upper-triangular subgroup of $\mathrm{O}(J)\left(k_{F}\right)$. (Here $\mathrm{O}(J)$ denotes the orthogonal group of the symmetric bilinear form defined by $J$.) The group $\mathfrak{B}$ contains an Iwahori subgroup with index 2 (pre-image of the upper-triangular subgroup of $\mathrm{SO}_{n}\left(k_{F}\right) \subset \mathcal{U}_{n}\left(k_{F}\right) \cong \mathrm{O}_{n}\left(k_{F}\right)$, see $[$ Tit79, 3.7]).

We define $\mathfrak{P}=U_{n}^{\mathcal{F}}$, where $\mathcal{F}$ is the subset of $A$ defined by the conditions

$$
\mathcal{F}: 0=a_{k}<a_{k-1}<\cdots<a_{1}<\frac{1}{4} .
$$

By [Tit79, 3.1], it is identified with the pre-image in $K$ under the canonical reduction map $\mathcal{U}_{n}\left(\mathcal{O}_{F}\right) \rightarrow$ $\mathcal{U}_{n}\left(k_{F}\right) \rightarrow \mathrm{O}(J)\left(k_{F}\right)$ of the matrices in $\mathrm{O}(J)\left(k_{F}\right)$ which are block upper-triangular, with blocks of size $1+\cdots+1+3+1+\cdots+1$. We see from the definitions that $[\mathfrak{P}: \mathfrak{B}]=q+1$ (observe that $U_{n}$ is residually split).

We let $B$ denote the upper-triangular Borel subgroup of $U_{n}$, and $P$ the parabolic subgroup generated by $B$ and the root subgroup corresponding to $a_{-k}$. We write $B=T N_{0}$ and $P=M N_{P}$ for the Levi decompositions of $B$ and $P$ with respect to $S$; thus $T=Z$ and the derived subgroup of $M$ is isomorphic to $\mathrm{SU}_{3}$.

Lemma 2.1. Let $\pi$ be an admissible $\mathbb{C}\left[U_{n}\right]$-module. Then there are canonical isomorphisms $\pi^{\mathfrak{B}} \cong \pi_{N_{0}}^{Z_{c}}$ and $\pi^{\mathfrak{P}} \cong \pi_{N_{P}}^{M \cap \mathfrak{P}}$, where the subscript indicates unnormalized Jacquet module.

Proof. The first isomorphism is [Cas80, Proposition 2.4]. The second follows from the first in the same way as in [CT15, Proposition 2.1].

We write $H_{\mathfrak{B}}$ for the convolution algebra of $\mathfrak{B}$-biinvariant functions $f: U_{n} \rightarrow \mathbb{Z}$, the Haar measure on $G$ being normalized so that $\mathfrak{B}$ has measure 1 . Thus $H_{\mathfrak{B}}$ is a free $\mathbb{Z}$-module, a basis being given by the double cosets $[\mathfrak{B} g \mathfrak{B}]$ for $g \in G ;[\mathfrak{B}]$ is the unit element. If $R$ is a ring then we define $H_{\mathfrak{B}, R}=H_{\mathfrak{B}} \otimes_{\mathbb{Z}} R$. As the extended affine Weyl group of $U_{n}$ equals the affine Weyl group, it follows from [Tit79, 3.1.1] that the triple $\left(U_{n}, \mathfrak{B}, N\right)$ is a Tits system (or BN-pair). Consequently, the Hecke algebra $H_{\mathfrak{B}}$ admits the following explicit description [Mat64, Théorème 4].

The affine Weyl group $W$ is generated by the set $\Sigma=\left\{s_{0}, \ldots, s_{k}\right\}$ in the simple affine roots, and the pair $(W, \Sigma)$ is an affine Coxeter group of type $\mathrm{B}_{k}$. There is a natural length function $l: W \rightarrow \mathbb{N}$, which takes a Weyl element $w \in W$ to the length of the shortest word in the elements of $\Sigma$ representing $w$. For each $w \in W$, the index $q_{w}=\# B w B / B$ equals $q^{l(w)}$ (see [Tit79, 3.3.1]). The associated braid group $B_{W}$ is defined as the quotient of the free group in the elements $T_{w}, w \in W$, by the relations $T_{w w^{\prime}}=T_{w} T_{w^{\prime}}$ if 
$l\left(w w^{\prime}\right)=l(w)+l\left(w^{\prime}\right)$. The Hecke algebra $H_{\mathfrak{B}}$ is isomorphic to the quotient of the algebra $\mathbb{Z}\left[B_{W}\right]$ by the relations $\left(T_{s}-1\right)\left(T_{s}+q\right)=0, s \in \Sigma$, the isomorphism being given by the formula $[\mathfrak{B} w \mathfrak{B}] \mapsto T_{w}$.

As in $[\mathrm{CT} 15, \S 2]$, we now study the algebra $H_{\mathfrak{B}}$ by identifying it with the Iwahori-Hecke algebra of a split reductive group over $F$. Let $\mathrm{Sp}_{2 k}$ denote the symplectic group over $F$ in $2 k=n-1$ variables over $F$, defined by the form

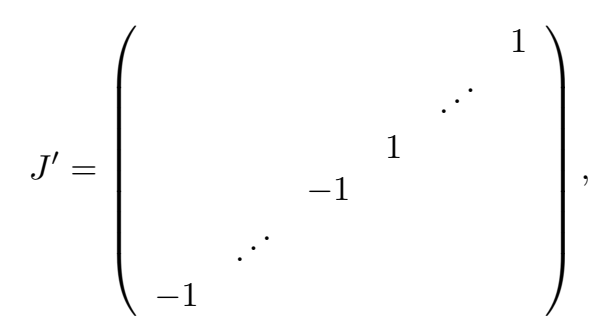

and let $S^{\prime} \subset \mathrm{Sp}_{2 k}$ denote the maximal $F$-split torus consisting of matrices

$$
\left(t_{1}, \ldots, t_{k}, t_{-k}, \ldots, t_{-1}\right), t_{i} \in F^{\times}, t_{i} t_{-i}=1 \text { for each } i=1, \ldots, k .
$$

Then $S^{\prime}$ is also a maximal torus of $\mathrm{Sp}_{2 k}$. Let $S_{c}^{\prime} \subset S^{\prime}$ denote the maximal compact subgroup, and let $\Lambda^{\prime}=S^{\prime} / S_{c}^{\prime}$. Then $\Lambda^{\prime} \cong \mathbb{Z}^{k}$, a basis being given by the elements

$$
\epsilon_{i}^{\prime}=\left(1, \ldots, \varpi^{2}, \ldots, \varpi^{-2}, \ldots, 1\right),
$$

where $\varpi^{2}$ occupies the $i$-entry. We identify $\Lambda \cong \Lambda^{\prime}$ via the isomorphism which sends $\epsilon_{i}$ to $\epsilon_{i}^{\prime}$ for each $i=1, \ldots, k$.

Let $W_{0}^{\prime}$ denote the Weyl group of $S^{\prime}$, and let $N^{\prime}=N_{\mathrm{Sp}_{2 k}}\left(S^{\prime}\right), W^{\prime}=N^{\prime} / S_{c}^{\prime}$. Let $\mathfrak{B}^{\prime}$ denote the open compact subgroup of $\mathrm{Sp}_{2 k}$ consisting of matrices with integral entries which are upper-triangular modulo the maximal ideal of $\mathcal{O}_{F}$. Then $\mathfrak{B}^{\prime}$ is an Iwahori subgroup of $\mathrm{Sp}_{2 k}$, and we write $H_{\mathfrak{B}^{\prime}}$ for its associated Hecke algebra. We write $K^{\prime} \subset \mathrm{Sp}_{2 k}$ for the subgroup of matrices with integral entries; it is a hyperspecial maximal compact subgroup of $\mathrm{Sp}_{2 k}$, and there is a corresponding semi-direct product decomposition $W^{\prime} \cong W_{0}^{\prime} \ltimes \Lambda^{\prime}$.

Proposition 2.2. There is an isomorphism $H_{\mathfrak{B}} \cong H_{\mathfrak{B}^{\prime}}$ of Hecke algebras sending $\left[\mathfrak{B} \epsilon_{i} \mathfrak{B}\right]$ to $\left[\mathfrak{B}^{\prime} \epsilon_{i}^{\prime} \mathfrak{B}^{\prime}\right]$ for each $i=1, \ldots, k$.

Proof. Using the identifications $W \cong W_{0} \ltimes \Lambda, W^{\prime} \cong W_{0}^{\prime} \ltimes \Lambda^{\prime}$, we can extend the isomorphisms $\Lambda \cong \Lambda^{\prime}$, $W_{0} \cong W_{0}^{\prime}$ to an isomorphism $W \cong W^{\prime}$. We must check that this isomorphism sends $\Sigma \subset W$ to a set of simple reflections $\Sigma^{\prime} \subset W^{\prime}$ corresponding to a choice of basis of affine roots. Indeed, the triple $\left(\operatorname{Sp}_{2 k}, \mathfrak{B}^{\prime}, N^{\prime}\right)$ is a Tits system (because $\mathrm{Sp}_{2 k}$ is simply connected). Appealing again to [Mat64], we then see that the isomorphism $f: W \cong W^{\prime}$ then determines an isomorphism $B_{W} \cong B_{W^{\prime}}$ of braid groups which gives, by passage to quotient, an isomorphism $H_{\mathfrak{B}} \cong H_{\mathfrak{B}^{\prime}}$, which sends $[\mathfrak{B} w \mathfrak{B}]$ to $\left[\mathfrak{B}^{\prime} f(w) \mathfrak{B}^{\prime}\right]$.

To do this check, we note that $\mathfrak{B}^{\prime}$ acts trivially on a unique chamber $C^{\prime}$ in the standard apartment $A\left(\mathrm{Sp}_{2 k}, S^{\prime}, F\right)$. This apartment admits a simple description, because $\mathrm{Sp}_{2 k}$ is split (see [Tit79, 1.1]), and it is easy to check that the isomorphism $W \cong W^{\prime}$ sends $\left\{s_{0}, \ldots, s_{k}\right\}$ to the set of simple reflections corresponding to the basis of affine roots corresponding to $C^{\prime}$. (The main point is that $s_{0}$ is mapped to the simple reflection in $W^{\prime}-W_{0}^{\prime}$, and this follows from the formula $s_{0}=s_{a_{1}} \cdot \epsilon_{1}$, consequence of (2.1).)

We now identify $W_{0}=W_{0}^{\prime}$ and $\Lambda=\Lambda^{\prime}$ and introduce the Bernstein presentation of the algebra $H_{\mathfrak{B}, \mathbb{C}} \cong H_{\mathfrak{B}^{\prime}, \mathbb{C}}$, following Lusztig [Lus89, $\S 3$. It is defined in terms of a based root datum $(X, Y, R, \check{R}, \Pi)$. We take $X=\Lambda, Y=\operatorname{Hom}(\Lambda, \mathbb{Z})$. We take $R$ to be the set of roots

$$
\left\{ \pm \epsilon_{i} \pm \epsilon_{j} \mid 1 \leq i<j \leq k\right\} \cup\left\{ \pm \epsilon_{i} \mid 1 \leq i \leq k\right\}
$$

and $\check{R}$ to be the set of coroots

$$
\left\{ \pm f_{i} \pm f_{j} \mid 1 \leq i<j \leq k\right\} \cup\left\{ \pm 2 f_{i} \mid 1 \leq i \leq k\right\}
$$


where $f_{1}, \ldots, f_{k}$ is the basis of $Y$ dual to the basis $\epsilon_{1}, \ldots, \epsilon_{k}$ of $X$. We choose the set $\Pi$ of simple roots to consist of the elements

$$
\beta_{i}=\epsilon_{i}-\epsilon_{i+1}, 1 \leq i \leq k-1, \text { and } \beta_{k}=\epsilon_{k} .
$$

This is the based root datum of the group $\mathrm{SO}_{2 k+1}(\mathbb{C})$, the Langlands dual of the simply connected group $\mathrm{Sp}_{2 k}$. In terms of this data, Lusztig defines in [Lus89, §3.2] a Hecke algebra $H$, which is the quotient of the group algebra $\mathbb{C}\left[v, v^{-1}\right]\left[B_{W}\right]$ by the relations $\left(T_{s}+1\right)\left(T_{s}-v^{2}\right)(s \in \Sigma)$. It follows from the above discussion that this algebra becomes canonically identified with $H_{\mathfrak{B}, \mathbb{C}}$ after specializing to the value $v=q^{1 / 2}$. The analysis in [Lus89] therefore applies to our Hecke algebra of interest.

This analysis gives rise to an isomorphism, the Bernstein presentation, of the algebra $H_{\mathfrak{B}, \mathbb{C}}$ with the twisted tensor product

$$
H_{\mathfrak{B}, \mathbb{C}}=H_{0} \widetilde{\otimes}_{\mathbb{C}} \mathbb{C}[X],
$$

where $H_{0} \subset H_{\mathfrak{B}, \mathbb{C}}$ is the subalgebra spanned by the elements $T_{w}, w \in W_{0}$ (abstractly isomorphic to the group algebra $\left.\mathbb{C}\left[W_{0}\right]\right)$ and $\mathbb{C}[X]$ is the co-ordinate ring of the complex algebraic torus $\operatorname{Hom}\left(\Lambda, \mathbb{C}^{\times}\right)$. The twisted tensor product is the usual tensor product as complex vector spaces, with algebra structure being determined by the following commutation relation. If $\beta \in \Pi$ is a simple root and $s=s_{\beta} \in W_{0}$ is the corresponding simple reflection, then $T_{s} \in H_{0}$, hence $B_{s}=T_{s}-q \in H_{0}$. If $\theta \in \mathbb{C}[X]$, then the following relation holds:

$$
\theta B_{s}=B_{s} \theta^{s}+\left(\theta^{s}-\theta\right) \zeta_{\beta},
$$

where $\zeta_{\beta}=\left(q-e_{\beta}\right) /\left(1-e_{\beta}\right)$ if $\beta$ is a long root, and $\zeta_{\beta}=\left(q-e_{\beta}\right)\left(1+e_{\beta}\right) /\left(1-e_{2 \beta}\right)$ otherwise. (Here we write $e_{\lambda} \in \mathbb{C}[X]$ for the basis element corresponding to $\lambda \in \Lambda$, and $W_{0}$ acts on $\mathbb{C}[X]$ via its natural right action.)

\subsection{Functoriality and base change for the group $U_{n}$}

We continue with the notation of the previous section. We now recall some elements of the theory of the Langlands parameterization; we will take these ideas up again in $\$ 3.1$ below, in a more general context. If $G$ is a reductive $F$-group, we write $\Pi(G)$ for the set of isomorphism classes of irreducible admissible $\mathbb{C}[G]$ modules, and $\Pi_{\text {temp }}(G)$ for the subset of isomorphism classes of tempered modules. We recall that thanks to the work of Moeglin [Mœg07] and Mok [Mok15] there is a decomposition of $\Pi_{\text {temp }}\left(U_{n}\right)$ as a disjoint union of sets $\Pi_{\varphi}$, as $\varphi$ ranges over conjugacy classes of Langlands parameters

$$
W_{F} \times \mathrm{SU}_{2}(\mathbb{R}) \rightarrow{ }^{L} U_{n} .
$$

Restriction to the subgroup $W_{E} \times \mathrm{SU}_{2}(\mathbb{R})$ induces a bijection between the set of these conjugacy classes, and the set of conjugacy classes of parameters

$$
W_{E} \times \mathrm{SU}_{2}(\mathbb{R}) \rightarrow \mathrm{GL}_{n}(\mathbb{C})
$$

which are conjugate orthogonal; see [Mok15, Lemma 2.2.1]. In particular, there is a map $\Pi_{\text {temp }}\left(U_{n}\right) \rightarrow$ $\Pi_{\text {temp }}\left(\mathrm{GL}_{n, E}\right)$, with image given by those representations corresponding to conjugate orthogonal parameters. We say that such a representation $\pi$ of $\mathrm{GL}_{n, E}$ is in the image of the stable base change map. The fibres of the map $\Pi_{\text {temp }}\left(U_{n}\right) \rightarrow \Pi_{\text {temp }}\left(\mathrm{GL}_{n, E}\right)$ are the L-packets $\Pi_{\varphi}$.

We are interested in a particular L-packet. Suppose that $n \geq 7$, and let $\Pi_{E}=\mathrm{St}_{n-4, E} \boxplus \mathrm{St}_{3, E} \boxplus \mathrm{St}_{1, E}$, a tempered representation of $\mathrm{GL}_{n, E}$. Let $\varphi_{E}$ denote the corresponding parameter. It extends uniquely to a parameter $\varphi$ of the previous type. Recall that there is a correspondence $f_{E} \sim f$ between smooth, compactly supported functions $f_{E}$ on $U_{n}(E)$ and $f$ on $U_{n}$, associated to stable base change. In the statement of the following theorem, we write $\left[m_{1}, \ldots, m_{k}\right]$ for the character $|\cdot|^{m_{1}} \otimes|\cdot|^{m_{2}} \otimes \cdots \otimes|\cdot|^{m_{k}} \otimes 1$ of the group $E^{\times} \times \cdots \times E^{\times} \times\left(E^{\times}\right)_{\mathbb{N}_{E / F}=1}$, the $F$-points of the maximal torus $T=Z_{U_{n}}(S) \subset U_{n}$. We will call such a character (generally occurring in a Jacquet module) an exponent. We write (?) ${ }_{N_{0}}^{\text {norm }}$ for the normalized Jacquet module with respect to the unipotent radical of the upper-triangular Borel subgroup of $U_{n}$.

Theorem 2.3. 1. Suppose that $n=7$. Then $\Pi_{\varphi}$ contains exactly two elements $X, Y$, which are uniquely characterized by the following properties: 
(a) $\langle\operatorname{tr} X+\operatorname{tr} Y, f\rangle=\left\langle\operatorname{tr} \Pi_{E} \times I_{\theta}, f_{E}\right\rangle$, where $I_{\theta}: \Pi_{E} \cong \Pi_{E}^{c}$ is the Whittaker-normalized intertwining operator.

(b) $\operatorname{dim} X^{\mathfrak{P}}=\operatorname{dim} X^{\mathfrak{B}}=1$ and $\operatorname{dim} Y^{\mathfrak{B}}=7$.

(c) $X_{N_{0}}^{\text {norm }}=[1,0,-1]$ and $\left(Y_{N_{0}}^{\text {norm }}\right)^{s s}=[1,0,-1]+2[1,0,1]+4[1,1,0]$.

2. Suppose that $n=9$. Then $\Pi_{\varphi}$ is a discrete L-packet and contains exactly four elements $X, Y, Z, W$, which are uniquely characterized by the following properties:

(a) $\langle\operatorname{tr} X+\operatorname{tr} Y+\operatorname{tr} Z+\operatorname{tr} W, f\rangle=\left\langle\operatorname{tr} \Pi_{E} \times I_{\theta}, f_{E}\right\rangle$, where $I_{\theta}: \Pi_{E} \cong \Pi_{E}^{c}$ is the Whittaker-normalized intertwining operator.

(b) $\operatorname{dim} X^{\mathfrak{P}}=\operatorname{dim} X^{\mathfrak{B}}=1, \operatorname{dim} Y^{\mathfrak{B}}=11, \operatorname{dim} Z^{\mathfrak{B}}=2$, and $\operatorname{dim} W^{\mathfrak{B}}=0$.

(c) We have

$$
\begin{aligned}
X_{N_{0}}^{\text {norm }} & =[2,1,0,-1] \\
\left(Y_{N_{0}}^{\text {norm }}\right)^{s s} & =[2,1,0,-1]+2[2,1,0,1]+4[2,1,1,0]+[1,0,2,1]+[1,2,0,1]+2[1,2,1,0] \\
\left(Z_{N_{0}}^{\text {norm }}\right)^{s s} & =[1,0,2,1]+[1,2,0,1] .
\end{aligned}
$$

3. Suppose that $n=2 k+1 \geq 11$. Then $\Pi_{\varphi}$ is a discrete L-packet and contains exactly four elements $X_{k}$, $Y_{k}, Z_{k}, W_{k}$, which are uniquely characterized by the following (inductively defined) properties:

(a) $\left\langle\operatorname{tr} X_{k}+\operatorname{tr} Y_{k}+\operatorname{tr} Z_{k}+\operatorname{tr} W_{k}, f\right\rangle=\left\langle\operatorname{tr} \Pi_{E} \times I_{\theta}, f_{E}\right\rangle$, where $I_{\theta}: \Pi_{E} \cong \Pi_{E}^{c}$ is the Whittaker-normalized intertwining operator.

(b) $\operatorname{dim} X_{k}^{\mathfrak{P}}=\operatorname{dim} X_{k}^{\mathfrak{B}}=1, \operatorname{dim} Y_{k}^{\mathfrak{B}}=\operatorname{dim} Y_{k-1}^{\mathfrak{B}}+k, \operatorname{dim} Z_{k}^{\mathfrak{B}}=\operatorname{dim} Z_{k-1}^{\mathfrak{B}}+(k-2)$, and $\operatorname{dim} W_{k}^{\mathfrak{B}}=0$.

(c) The exponents of the Jacquet modules of these representations are as follows.

i. $X_{k, N_{0}}^{\text {norm }}=[k-2, k-3, \ldots, 0,-1]$.

ii. $\left(Y_{k, N_{0}}^{\text {norm }}\right)^{s s}$ is the sum of the exponents $[1, k-2, k-3, \ldots, 1]$ with 0 inserted as the $i$ entry for $i=2, \ldots, k-1$, the exponent $2[1, k-2, k-3, \ldots, 0]$, together with the exponents of $\left(Y_{k-1, N_{0}}^{n o r m}\right)^{s s}$ with $k-2$ inserted as the first entry.

iii. $\left(Z_{k, N_{0}}^{\text {norm }}\right)^{\text {ss }}$ is the sum of the exponents $[1, k-2, k-3, \ldots, 1]$ with 0 inserted as the $i$ entry for $i=2, \ldots, k-1$, together with the exponents of $\left(Z_{k-1, N_{0}}^{n o r m}\right)^{s s}$ with $k-2$ inserted as the first entry.

The proof of Theorem 2.3 will be given in several stages. Kazhdan and Lusztig have proved [KL87] for certain groups what they call the Deligne-Langlands conjecture, which includes as a special case Theorem 2.4 below. We first apply their construction to obtain Theorem 2.5, which asserts the existence of semi-stable admissible $\mathbb{C}\left[U_{n}\right]$-modules $X, Y$ and (if $n \geq 9$ ) $Z$, defined in terms of the Langlands parameter $\varphi_{E}$, and with Jacquet modules as described by Theorem 2.3 above. It seems reasonable to guess that these modules are the semi-stable modules in the L-packet corresponding to the Langlands parameter. However, this requires proof, and does not follow immediately from the construction of Kazhdan-Lusztig. (Indeed, our L-packets are characterized by an identity of stable traces; since the construction of Kazhdan-Lusztig yields only the representations in the packet with non-zero Iwahori invariants, some further argument must be required.)

In $\S 3$ we will describe, following [Mok15], the endoscopic classification of representations of the group $U_{n}$, and describe in more detail the L-packets of representations corresponding under this classification to the parameter $\varphi_{E}$. We will then verify in $\S 3.5$ that the representations constructed in Theorem 2.5 do indeed appear in this L-packet, and thus deduce Theorem 2.3.

Theorem 2.4. Let $G$ be a split reductive group over $F$, with connected center, and let $\mathfrak{I} \subset G$ be an Iwahori subgroup. Then the following two sets are in natural bijection:

1. The set of representations $\pi \in \Pi_{\text {temp }}(G)$ such that $\pi^{\mathfrak{I}} \neq 0$.

2. The set of $\widehat{G}$-conjugacy classes of triples $(s, u, \rho)$, where: 
(a) $s \in \widehat{G}$ is a semi-simple element contained in a maximal compact subgroup of $\widehat{G}(\mathbb{C})$.

(b) $u \in \widehat{G}$ is a unipotent element such that sus $^{-1}=u^{q}$.

(c) Let $\mathcal{B}_{u}^{s}$ be the variety of Borel subgroups $B \subset \widehat{G}$ such that $s \in B$ and $u \in B$. Let $\bar{M}(u, s)=$ $\pi_{0}\left(\left(Z_{\widehat{G}}(s) \cap Z_{\widehat{G}}(u)\right) / Z_{\widehat{G}}\right)$. Then $\rho$ is an irreducible representation of $\bar{M}(u, s)$ such that

$$
\operatorname{Hom}_{\mathbb{C}[\bar{M}(u, s)]}\left(\rho, H_{*}\left(\mathcal{B}_{u}^{s}, \mathbb{C}\right)\right) \neq 0 .
$$

Proof. The existence of the bijection is [KL87, Theorem 7.12]; its particular properties in the tempered case are contained in [KL87, Theorem 8.2]. The relevant Hecke-modules are defined in terms of equivariant K-homology, but can be recovered as complex vector spaces as the usual singular homology with complex coefficients, cf. [Ree94, §2]. For a description of these modules directly in terms of (Borel-Moore) homology, see [CG10, Ch. 8].

Theorem 2.5. 1. Suppose that $n=7$. Then there exist irreducible admissible $\mathbb{C}\left[U_{n}\right]$-modules $X, Y$ with the following properties.

(a) $\operatorname{dim} X^{\mathfrak{P}}=\operatorname{dim} X^{\mathfrak{B}}=1$ and $\operatorname{dim} Y^{\mathfrak{B}}=7$.

(b) $X_{N_{0}}^{\text {norm }}=[1,0,-1]$ and $\left(Y_{N_{0}}^{\text {norm }}\right)^{s s}=[1,0,-1]+2[1,0,1]+4[1,1,0]$.

2. Suppose that $n=9$. Then there exist irreducible admissible $\mathbb{C}\left[U_{n}\right]$-modules $X, Y$, and $Z$ with the following properties:

(a) $\operatorname{dim} X^{\mathfrak{P}}=\operatorname{dim} X^{\mathfrak{B}}=1$, $\operatorname{dim} Y^{\mathfrak{B}}=11$, and $\operatorname{dim} Z^{\mathfrak{B}}=2$.

(b) We have

$$
\begin{aligned}
X_{N_{0}}^{\text {norm }} & =[2,1,0,-1] \\
\left(Y_{N_{0}}^{\text {nom }}\right)^{s s} & =[2,1,0,-1]+2[2,1,0,1]+4[2,1,1,0]+[1,0,2,1]+[1,2,0,1]+2[1,2,1,0] \\
\left(Z_{N_{0}}^{\text {norm }}\right)^{s s} & =[1,0,2,1]+[1,2,0,1] .
\end{aligned}
$$

3. Suppose that $n=2 k+1 \geq 11$. Then there exist irreducible admissible $\mathbb{C}\left[U_{n}\right]$-modules $X_{k}, Y_{k}$, and $Z_{k}$, with the following properties:

(a) $\operatorname{dim} X_{k}^{\mathfrak{P}}=\operatorname{dim} X_{k}^{\mathfrak{B}}=1, \operatorname{dim} Y_{k}^{\mathfrak{B}}=\operatorname{dim} Y_{k-1}^{\mathfrak{B}}+k$, and $\operatorname{dim} Z_{k}^{\mathfrak{B}}=\operatorname{dim} Z_{k-1}^{\mathfrak{B}}+(k-2)$.

(b) The exponents of the Jacquet modules of these representations are as follows.

i. $X_{k, N_{0}}^{n o r m}=[k-2, k-3, \ldots, 0,-1]$.

ii. $\left(Y_{k, N_{0}}^{\text {norm }}\right)^{\text {ss }}$ is the sum of the exponents $[1, k-2, k-3, \ldots, 1]$ with 0 inserted as the $i$ entry for $i=2, \ldots, k-1$, the exponent $2[1, k-2, k-3, \ldots, 0]$, together with the exponents of $\left(Y_{k-1, N_{0}}^{n o r m}\right)^{s s}$ with $k-2$ inserted as the first entry.

iii. $\left(Z_{k, N_{0}}^{\text {norm }}\right)^{\text {ss }}$ is the sum of the exponents $[1, k-2, k-3, \ldots, 1]$ with 0 inserted as the $i$ entry for $i=2, \ldots, k-1$, together with the exponents of $\left(Z_{k-1, N_{0}}^{\text {norm }}\right)^{s s}$ with $k-2$ inserted as the first entry.

Proof. All the representations we construct will have non-zero $\mathfrak{B}$-invariant vectors. Since we have identified (in Proposition 2.2) the Hecke algebra $H_{\mathfrak{B}, \mathbb{C}}$ with the Iwahori-Hecke algebra of a split group $\operatorname{Sp}_{2 k}(F)$, we can construct the desired $\mathbb{C}\left[U_{n}\right]$-modules by constructing representations of $\operatorname{Sp}_{2 k}(F)$ with non-zero Iwahorifixed vectors. We will in fact apply Theorem 2.4 to construct irreducible representations of $\operatorname{PSp}_{2 k}(F)$ with irreducible pullback to $\operatorname{Sp}_{2 k}(F)$. We fix a maximal torus $T \subset \operatorname{PSp}_{2 k}(F)$, and identify the dual group of $\mathrm{Sp}_{2 k}(F)$ as $\mathrm{SO}_{2 k+1}(\mathbb{C})$. To construct the data $(s, u)$, note that for each odd integer $i$ there is, up to isomorphism, a unique irreducible representation $V(i)$ of $\mathrm{SL}_{2}(\mathbb{C})$ of dimension $i$, which is orthogonal. We fix a choice of symmetric bilinear form on $V(i)$. Given a partition of $n=2 k+1=n_{1}+\cdots+n_{r}$ into odd integers, there is a homomorphism $\mathrm{SL}_{2}(\mathbb{C}) \rightarrow \mathrm{SO}_{2 k+1}(\mathbb{C})$, well-defined up to conjugacy in the latter group, giving the 
action of $\mathrm{SL}_{2}(\mathbb{C})$ on $V\left(n_{1}\right) \oplus \cdots \oplus V\left(n_{r}\right)$. This lifts uniquely to a homomorphism $\varphi: \mathrm{SL}_{2}(\mathbb{C}) \rightarrow \operatorname{Spin}_{2 k+1}(\mathbb{C})$. We associate to the partition the pair $(s, u)$ where

$$
s=\varphi\left(\begin{array}{cc}
q^{1 / 2} & 0 \\
0 & q^{-1 / 2}
\end{array}\right) \text { and } u=\varphi\left(\begin{array}{ll}
1 & 1 \\
0 & 1
\end{array}\right) .
$$

We now split into cases, as in the statement of the theorem.

1. The relevant partition is $7=3+3+1$. The variety $\mathcal{B}_{u}^{s}$ can be identified with the variety of isotropic flags $F^{\bullet}=\left(0 \subset F^{1} \subset F^{2} \subset F^{3}\right)$ in $\mathbb{C}^{7}$ such that $s\left(F^{i}\right) \subset F^{i}$ and $u\left(F^{i}\right) \subset F^{i}$ for each $i=1,2,3$. The group $\bar{M}(u, s)$ has two elements. Indeed, if we write $S=\mathrm{SL}_{2}(\mathbb{C})$ and $B_{S} \subset S$ for its standard upper-triangular Borel subgroup, then for any (algebraic) representation of $S$ on a finite-dimensional C-vector space $V$, we have $H^{0}(S, V)=H^{0}\left(B_{S}, V\right)$ and $\operatorname{End}_{S}(V)=\operatorname{End}_{B_{S}}(V)$. On the other hand, the elements

$$
s_{0}=\left(\begin{array}{cc}
q^{1 / 2} & 0 \\
0 & q^{-1 / 2}
\end{array}\right), u_{0}=\left(\begin{array}{cc}
1 & 1 \\
0 & 1
\end{array}\right)
$$

generate a Zariski dense subgroup of $B_{S}$. It follows that the groups $M(u, s)$ and $\bar{M}(u, s)$ can be computed in terms of the centralizer of the parameter $\varphi$, giving $\bar{M}(u, s) \cong\{ \pm 1\}$.

The variety $\mathcal{B}_{u}^{s}$ is isomorphic to the disjoint union of varieties $X_{1}, X_{2}, X_{3}$ on which $\bar{M}(u, s)$ acts. According to $[\mathrm{KL} 87, \S 7.6]$, we can associate a character $e_{\alpha}$ of the maximal torus $S^{\prime}$ of $\operatorname{Sp}_{2 k}(F)$ to each $\bar{M}(u, s)$-orbit $\mathcal{B}^{\alpha}$ of connected components of $\mathcal{B}_{u}^{s}$. The semi-simplification of the Jacquet module of the representation associated to a triple $(u, s, \rho)$ is then $\oplus_{\alpha} \operatorname{dim}_{\mathbb{C}} \operatorname{Hom}_{\bar{M}(u, s)}\left(\rho, H_{*}\left(\mathcal{B}^{\alpha}, \mathbb{C}\right)\right) \cdot e_{\alpha}$. The varieties $X_{i}$ and their associated exponents are as follows:

(a) $X_{1}$ is a union of two points which are interchanged by $\bar{M}(u, s)$. The associated exponent is $[1,0,-1]$.

(b) $X_{2} \cong \mathbb{P}^{1}$. The associated exponent is $[1,0,1]$.

(c) $X_{3} \cong \mathbb{P}^{1} \times \mathbb{P}^{1}$. The associated exponent is $[1,1,0]$.

In general, calculating explicitly the varieties $\mathcal{B}_{u}^{s}$ is a difficult problem; see [DCLP88]. We now describe how one can obtain the above description in this case. Let $D \subset \widehat{G}=\operatorname{Spin}_{2 k+1}(\mathbb{C})$ denote the image under $\varphi$ of the diagonal torus in $\mathrm{SL}_{2}$, and let $T \subset \widehat{G}$ denote the connected centralizer of the image of $\varphi$. Then $T$ is a rank 1 torus, and $D \times T$ is regular, in the sense that its centralizer in $\widehat{G}$ is a maximal torus. In particular, the eigenspaces of $D \times T$ in the standard $n$-dimensional representation of $\widehat{G}$ are 1-dimensional.

Let $\mathcal{B}_{u} \subset \mathcal{B}$ denote the variety of Borel subgroups containing the unipotent element $u$. Let $\mathcal{B}_{u}^{D} \subset \mathcal{B}_{u}$ denote the $D$-fixed subvariety, and $\mathcal{B}_{u}^{D, T}$ the $D \times T$-fixed subvariety. Then $\mathcal{B}_{u}^{D}$ is smooth and projective, for general reasons; see [DCLP88, §3.6]. On the other hand, we have $\mathcal{B}_{u}^{D}=\mathcal{B}_{u}^{s}$, hence $\mathcal{B}_{u}^{s}$ is smooth and projective.

We now apply the theorem of Bialynicki-Birula (cf. [CG10, Theorem 2.4.3]) to the action of the torus $T$ on the smooth, projective variety $\mathcal{B}_{u}^{D}$. It is easy to show that $\mathcal{B}_{u}^{D, T}$ is a finite set, containing 8 elements. Indeed, if $F^{\bullet} \in \mathcal{B}_{u}^{D, T}$, then each $F^{i}$ must be a sum of $D \times T$-eigenspaces. It follows that $\mathcal{B}_{u}^{D}$ has a paving by affine spaces $X_{w}$, indexed by elements $w \in \mathcal{B}_{u}^{D, T}$. This immediately implies that $H_{*}\left(\mathcal{B}_{u}^{s}, \mathbb{C}\right)$ is 8-dimensional, and that the exponents of the Jacquet module of $X+Y$, taken with their multiplicities, are as claimed in the theorem. One can now compute the cells $X_{w}$ explicitly, together with the action of the group $\bar{M}(u, s)$; this gives the varieties described above.

2. The relevant partition is $9=5+3+1$. The group $\bar{M}(u, s)$ is isomorphic to $\{ \pm 1\} \times\{ \pm 1\}$. We can choose distinct non-trivial characters $\epsilon_{X}, \epsilon_{Z}$ of this group so that the variety $\mathcal{B}_{u}^{s}$ is isomorphic to a union of varieties $X_{1}, \ldots, X_{6}$ on which the group $\bar{M}(u, s)$ acts, and having the following properties:

(a) $X_{1}$ and $X_{2}$ are each a union of two points, which are interchanged by the quotient of $\bar{M}(u, s)$ by ker $\epsilon_{Z}$. The associated exponents are, respectively, $[1,0,2,1]$ and $[1,2,0,1]$. 
(b) $X_{3}$ is isomorphic to $\mathbb{P}^{1}$. The associated exponent is $[1,2,1,0]$.

(c) $X_{4}$ is a union of two points, which are interchanged by the quotient of $\bar{M}(u, s)$ by ker $\epsilon_{X}$. The associated exponent is $[2,1,0,-1]$.

(d) $X_{5}$ is isomorphic to $\mathbb{P}^{1}$. The associated exponent is $[2,1,0,1]$.

(e) $X_{6}$ is isomorphic to $\mathbb{P}^{1} \times \mathbb{P}^{1}$. The associated exponent is $[2,1,1,0]$.

This description of the variety $\mathcal{B}_{u}^{s}$ can be obtained by applying the inductive procedure of [Ree94, pp. 485-486] and [DCLP88, §3.8].

3. The relevant partition is $n=(n-4)+3+1$. The group $\bar{M}(u, s)$ is isomorphic to $\{ \pm 1\} \times\{ \pm 1\}$. We can choose distinct non-trivial characters $\epsilon_{X}, \epsilon_{Z}$ of this group so that the variety $\mathcal{B}_{u}^{s}$ is isomorphic to a union of varieties $X_{1}, \ldots, X_{k-1}$ and $\mathcal{B}_{u}^{s}(k-1)$ (i.e. the variety associated to the group $\operatorname{PSp}_{2(k-1)}$ ) and having the following properties.

(a) The varieties $X_{1}, \ldots, X_{k-2}$ are each isomorphic to a union of two points, which are interchanged by the quotient of $\bar{M}(u, s)$ by $\operatorname{ker} \epsilon_{Z}$. The associated exponent of $X_{i}$ is $[1, k-2, k-3, \ldots, 1]$, with an extra 0 inserted as the $i+1$ entry.

(b) The variety $X_{k-1}$ is isomorphic to $\mathbb{P}^{1}$. The associated exponent is $[1, k-2, k-3, \ldots, 1,0]$.

(c) The action on $\mathcal{B}_{u}^{s}(k-1)$ is the one constructed by induction. The associated exponents are those of the variety $\mathcal{B}_{u}^{s}(k-1)$, with $k-2$ inserted as the first entry. (Compare the statement of the theorem.)

This description of the variety $\mathcal{B}_{u}^{s}$ can be obtained by applying the inductive procedure of [Ree94, pp. 485-486] and [DCLP88, §3.8].

Finally, we have to check that $\operatorname{dim} X^{\mathfrak{P}}=1$. By Lemma 2.1, this follows from the computation of $X_{N_{0}}^{\text {norm }}$ and the fact that the representation of $U(3)$ induced from the character [-1] has the trivial representation as its unique submodule.

\subsection{Integral structures in semi-stable Hecke modules}

Let us now suppose that $l=n-2$ is prime, and fix an isomorphism $\iota: \overline{\mathbb{Q}}_{l} \cong \mathbb{C}$. Let $K \subset \overline{\mathbb{Q}}_{l}$ be a finite extension of $\mathbb{Q}_{l}$ over which all the representations $\iota^{-1} X, \iota^{-1} Y, \iota^{-1} Z, \iota^{-1} W$ (if the latter two exist) may be defined. (We recall that if $V$ is an irreducible admissible $\overline{\mathbb{Q}}_{l}\left[U_{n}\right]$-module, then $V^{\mathfrak{B}}$ is an irreducible $H_{\mathfrak{B}, \overline{\mathbb{Q}}_{l}}$ module; we can therefore find a finite extension $K / \mathbb{Q}_{l}$ and a $H_{\mathfrak{B}, K}$-module $M \subset V^{\mathfrak{B}}$ such that the natural map $M \otimes_{K} \overline{\mathbb{Q}}_{l} \rightarrow V^{\mathfrak{B}}$ is an isomorphism. The procedure of [BH06, 4.3, Proposition] then gives a model of $V$ which is defined over $K$.) We assume that $K$ contains a square root of $q$. We write $X_{K}, Y_{K}$ etc. for a choice of $K\left[U_{n}\right]$-modules realizing this descent.

Let $\mathcal{O}$ denote the ring of integers of $K, \lambda \subset \mathcal{O}$ the maximal ideal, $k=\mathcal{O} / \lambda$ the residue field. As explained in the introduction, the proofs of our main theorems are restricted to the cases $l=5$ and $l=7$. Therefore, the following computations have been completed only in these cases.

Proposition 2.6. $\quad$ 1. Suppose that $n=7$, and that $q$ is a primitive root modulo $l$. Then there exist $H_{\mathfrak{B}, \mathcal{O}}$-submodules $X_{\mathcal{O}}^{\mathfrak{B}} \subset X_{K}^{\mathfrak{B}}$ and $Y_{\mathcal{O}}^{\mathfrak{B}} \subset Y_{K}^{\mathfrak{B}}$ which are finite $\mathcal{O}$-modules, and such that the natural maps

$$
X_{\mathcal{O}}^{\mathfrak{B}} \otimes_{\mathcal{O}} K \rightarrow X_{K}^{\mathfrak{B}} \text { and } Y_{\mathcal{O}}^{\mathfrak{B}} \otimes_{\mathcal{O}} K \rightarrow Y_{K}^{\mathfrak{B}}
$$

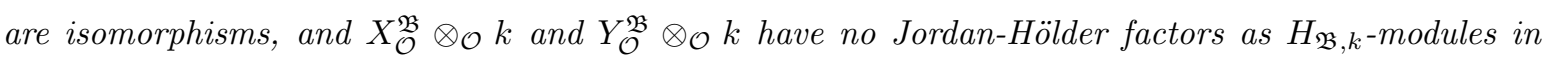
common.

2. Suppose that $n=9$, and that $q$ is a primitive root modulo $l$. Then there exist $H_{\mathfrak{B}, \mathcal{O}}$-submodules $X_{\mathcal{O}}^{\mathfrak{B}} \subset X_{K}^{\mathfrak{B}}, Y_{\mathcal{O}}^{\mathfrak{B}} \subset Y_{K}^{\mathfrak{B}}$, and $Z_{\mathcal{O}}^{\mathfrak{B}} \subset Z_{K}^{\mathfrak{B}}$ which are finite as $\mathcal{O}$-modules and such that the natural maps

$$
X_{\mathcal{O}}^{\mathfrak{B}} \otimes_{\mathcal{O}} K \rightarrow X_{K}^{\mathfrak{B}}, Y_{\mathcal{O}}^{\mathfrak{B}} \otimes_{\mathcal{O}} K \rightarrow Y_{K}^{\mathfrak{B}} \text { and } Z_{\mathcal{O}}^{\mathfrak{B}} \otimes_{\mathcal{O}} K \rightarrow Z_{K}^{\mathfrak{B}}
$$

are isomorphisms, and the sets of Jordan-Hölder factors of the representations $X_{\mathcal{O}}^{\mathfrak{B}} \otimes_{\mathcal{O}} k, Y_{\mathcal{O}}^{\mathfrak{B}} \otimes_{\mathcal{O}} k$ and $Z_{\mathcal{O}}^{\mathfrak{B}} \otimes_{\mathcal{O}} k$ have pairwise empty intersection. 
Proof. 1. Since $X_{K}^{\mathfrak{B}}$ is 1 -dimensional, we can choose $X_{\mathcal{O}}^{\mathfrak{B}}$ to be any $\mathcal{O}$-sublattice of $X_{K}^{\mathfrak{B}}$. To construct $Y_{\mathcal{O}}^{\mathfrak{B}}$, let us first consider the action of $H_{\mathfrak{B}, \mathcal{O}}$ on $M=\mathcal{O}^{7}$ given by the following matrices:

$$
T_{s_{1}}=\left(\begin{array}{ccccccc}
-1 & 1 & 0 & 0 & 0 & 0 & 0 \\
0 & q & 0 & 0 & 0 & 0 & 0 \\
0 & 0 & -1 & 1 & 0 & 0 & 0 \\
0 & 0 & 0 & q & 0 & 0 & 0 \\
0 & 0 & 0 & 0 & -1 & 0 & 0 \\
0 & 0 & 0 & 0 & 0 & -1 & 0 \\
0 & 0 & 0 & 0 & 0 & 0 & -1
\end{array}\right),
$$

and

$$
\begin{aligned}
\epsilon_{1}=\left(\begin{array}{ccccccc}
q^{-1} & 0 & 0 & 0 & 0 & 0 & 0 \\
1-q^{-1} & q^{-1} & 0 & 0 & 0 & 0 & 0 \\
0 & 0 & q^{-1} & 0 & 0 & 0 & 0 \\
0 & 0 & 1-q^{-1} & q^{-1} & 0 & 0 & 0 \\
0 & 0 & 0 & 0 & q^{-1} & 0 & 0 \\
0 & 0 & 0 & 0 & 2\left(1-q^{-1}\right) & q^{-1} & 0 \\
0 & 0 & 0 & 0 & 0 & 0 & q^{-1}
\end{array}\right) \\
\epsilon_{2}=\left(\begin{array}{cccccccc}
q^{-1} & 0 & 0 & 0 & 0 & 0 & 0 \\
-1+q^{-1} & q^{-1} & 0 & 0 & 0 & 0 & 0 \\
0 & 0 & q^{-1} & 0 & 0 & 0 & 0 \\
0 & 0 & -1+q^{-1} & q^{-1} & 0 & 0 & 0 \\
0 & 0 & 0 & 0 & 1 & 0 & 0 \\
0 & 0 & 0 & 0 & 2(q-1) & 1 & 0 \\
0 & 0 & 0 & 0 & 0 & 0 & 1
\end{array}\right) \\
\epsilon_{3}=\left(\begin{array}{cccccccc}
1 & 0 & 0 & 0 & 0 & 0 & 0 \\
0 & 1 & 0 & 0 & 0 & 0 & 0 \\
2(q-1) & 0 & 1 & 0 & 0 & 0 & 0 \\
0 & 2(q-1) & 0 & 1 & 0 & 0 & 0 \\
0 & 0 & 0 & 0 & q^{-1} & 0 & 0 \\
0 & 0 & 0 & 0 & 2\left(-1+q^{-1}\right) & q^{-1} & 0 \\
0 & 0 & 0 & 0 & 0 & 0 & q
\end{array}\right)
\end{aligned}
$$

(These matrices were calculated using the results of Reeder [Ree00], exactly as in the proof of [CT15, Proposition 2.9 ].) It follows from Reeder's construction that they satisfy the defining relations of the presentation $H_{\mathfrak{B}, \mathbb{C}}=H_{0} \widetilde{\otimes}_{\mathbb{C}} \mathbb{C}[X]$, so that $M$ is indeed a $H_{\mathfrak{B}, \mathcal{O}}$-module. The character $[1,1,0]$ of $K[X]$ visibly appears in $M \otimes_{\mathcal{O}} K$ with multiplicity 4 ; it also has multiplicity 4 in the full representation 
induced from this character of $T$. By Frobenius reciprocity, we must therefore have $M \otimes_{\mathcal{O}} K \cong Y_{K}^{\mathfrak{B}}$, as both modules are irreducible, and we can take $Y_{\mathcal{O}}^{\mathfrak{B}}$ to be the image of $M$ under such an isomorphism. To prove the claim about Jordan-Hölder factors, we now show that in fact the module $M \otimes_{\mathcal{O}} k$ is an absolutely irreducible $H_{\mathfrak{B}, k}$-module. This will use the assumption that $q$ is a primitive root modulo 5 . Let $V \subset M \otimes_{\mathcal{O}} k$ be a simple $H_{\mathfrak{B}, k}$-submodule; we will show that $V=M \otimes_{\mathcal{O}} k$. We can decompose $V=V_{[1,1,0]} \oplus V_{[1,0,1]} \oplus V_{[1,0,-1]}$ as a sum of generalised $k[X]$-eigenspaces; if $\mathbf{e}_{1}, \ldots, \mathbf{e}_{7}$ denotes the standard basis of $k^{7}=M \otimes_{\mathcal{O}} k$, then we have

$$
V_{[1,1,0]}=V \cap\left\langle\mathbf{e}_{1}, \ldots, \mathbf{e}_{4}\right\rangle, V_{[1,0,1]}=V \cap\left\langle\mathbf{e}_{5}, \mathbf{e}_{6}\right\rangle \text {, and } V_{[1,0,-1]}=V \cap\left\langle\mathbf{e}_{7}\right\rangle .
$$

Since $V$ is non-zero, at least one of these eigenspaces must be non-zero. We treat each possibility in turn. If $V_{[1,0,-1]} \neq 0$, then $\mathbf{e}_{7} \in V_{[1,0,-1]}$. The relations

$$
\mathbf{e}_{6}=T_{s_{3}} \mathbf{e}_{7}-q \mathbf{e}_{7}, \mathbf{e}_{4}=T_{s_{2}} \mathbf{e}_{6}-q \mathbf{e}_{6}, \mathbf{e}_{3}=T_{s_{1}} \mathbf{e}_{4}-q \mathbf{e}_{4}, \mathbf{e}_{1}=T_{s_{3}} \mathbf{e}_{3}-q \mathbf{e}_{3}
$$

show that $\left\langle\mathbf{e}_{1}, \mathbf{e}_{3}, \mathbf{e}_{4}, \mathbf{e}_{6}, \mathbf{e}_{7}\right\rangle \subset V$. The relations

$\mathbf{e}_{2}=\left(1-q^{-1}\right)^{-1}\left(\epsilon_{1} \mathbf{e}_{1}-q^{-1} \mathbf{e}_{1}\right), \mathbf{e}_{5}=q^{-1}(q+1)^{-1}\left(T_{s_{2}} \mathbf{e}_{1}+\mathbf{e}_{1}-q \mathbf{e}_{2}-2 q \mathbf{e}_{3}+2 q(q+1) \mathbf{e}_{4}+2 q\left(q^{2}+4 q+1\right) \mathbf{e}_{6}\right)$

then show that $V=M \otimes_{\mathcal{O}} k$.

Now suppose that $V_{[1,0,1]} \neq 0$. Looking at the action of $k[X]$, we see that that $\mathbf{e}_{6} \in V$, hence (using the above relations) $\left\langle\mathbf{e}_{1}, \ldots, \mathbf{e}_{6}\right\rangle \subset V$. Then the relation

$$
\mathbf{e}_{7}=(2 q(q+1))^{-1}\left(T_{s_{3}} \mathbf{e}_{5}+\mathbf{e}_{5}-2 q \mathbf{e}_{6}\right)
$$

shows $V=M \otimes_{\mathcal{O}} k$.

Now suppose finally that $V_{[1,1,0]} \neq 0$. Looking at the action of $k[X]$, we see that $\mathbf{e}_{4} \in V$, hence (using the above relations) $\mathbf{e}_{3} \in V$, hence $\mathbf{e}_{6}=(q(q+1))^{-1}\left(T_{s_{2}} \mathbf{e}_{3}+\mathbf{e}_{3}-q \mathbf{e}_{4}\right) \in V$, hence $V_{[1,0,1]} \neq 0$. This shows again $V=M \otimes_{\mathcal{O}} k$.

2. We can again choose $X_{\mathcal{O}}^{\mathfrak{B}}$ to be an arbitrary $\mathcal{O}$-lattice of $X_{K}^{\mathfrak{B}}$. We define an action of $H_{\mathfrak{B}, \mathcal{O}}$ on $M=\mathcal{O}^{11}$ via the following matrices:

$$
T_{s_{1}}=\left(\begin{array}{cccccccccccc}
-1 & 0 & 0 & 0 & 0 & 0 & 0 & 0 & 0 & 0 & 0 \\
q & -1 & 0 & 0 & 0 & 0 & 0 & 1 & 0 & 0 & 0 \\
0 & 0 & -1 & 0 & 0 & 0 & 0 & 0 & 0 & 0 & 0 \\
0 & 0 & q & -1 & 0 & 0 & 0 & 0 & 1 & 0 & 0 \\
0 & 0 & 0 & 0 & -1 & 0 & 0 & 0 & 0 & 0 & 0 \\
0 & 0 & 0 & 0 & 2 q & -1 & 0 & 0 & 0 & 1 & 0 \\
0 & 0 & 0 & 0 & 0 & 0 & -1 & 0 & 0 & 0 & 0 \\
q(1+q) & 0 & 0 & 0 & 0 & 0 & 0 & q & 0 & 0 & 0 \\
0 & 0 & q(1+q) & 0 & 0 & 0 & 0 & 0 & q & 0 & 0 \\
0 & 0 & 0 & 0 & 2 q(1+q) & 0 & 0 & 0 & 0 & q & 0 \\
0 & & 0 & 0 & 0 & 0 & 0 & 0 & 0 & 0 & 0 & -1
\end{array}\right),
$$




$$
\begin{aligned}
& T_{s_{3}}=\left(\begin{array}{ccccccccccc}
-1 & 0 & 0 & 0 & 0 & 0 & 0 & 0 & 0 & 0 & 0 \\
q & -1 & 0 & 0 & 1 & 0 & 0 & 0 & 0 & 0 & 0 \\
2 q & 0 & -1 & 0 & 2 & 0 & 0 & 0 & 0 & 0 & 0 \\
-2 q(1+q) & 2 q & q & -1 & 0 & 1 & 0 & 0 & 0 & 0 & 0 \\
q(1+q) & 0 & 0 & 0 & q & 0 & 0 & 0 & 0 & 0 & 0 \\
-2 q\left(1+4 q+q^{2}\right) & 2 q(1+q) & q(1+q) & 0 & -4 q & q & 0 & 0 & 0 & 0 & 0 \\
0 & 0 & 0 & 0 & 0 & 0 & -1 & 0 & 0 & 0 & 0 \\
0 & 0 & 0 & 0 & 0 & 0 & 0 & -1 & 0 & 0 & 0 \\
0 & 0 & 0 & 0 & 0 & 0 & 0 & 2 q & -1 & 1 & 0 \\
0 & 0 & 0 & 0 & 0 & 0 & 0 & 2 q(1+q) & 0 & q & 0 \\
0 & 0 & 0 & 0 & 0 & 0 & 0 & 0 & 0 & 0 & -1
\end{array}\right), \\
& T_{s_{4}}=\left(\begin{array}{ccccccccccc}
-1 & 0 & 1 & 0 & 0 & 0 & 0 & 0 & 0 & 0 & 0 \\
0 & -1 & 0 & 1 & 0 & 0 & 0 & 0 & 0 & 0 & 0 \\
0 & 0 & q & 0 & 0 & 0 & 0 & 0 & 0 & 0 & 0 \\
0 & 0 & 0 & q & 0 & 0 & 0 & 0 & 0 & 0 & 0 \\
0 & 0 & 0 & 0 & -1 & 0 & 0 & 0 & 0 & 0 & 0 \\
0 & 0 & 0 & 0 & 2 q & -1 & 1 & 0 & 0 & 0 & 0 \\
0 & 0 & 0 & 0 & 2 q(1+q) & 0 & q & 0 & 0 & 0 & 0 \\
0 & 0 & 0 & 0 & 0 & 0 & 0 & -1 & 1 & 0 & 0 \\
0 & 0 & 0 & 0 & 0 & 0 & 0 & 0 & q & 0 & 0 \\
0 & 0 & 0 & 0 & 0 & 0 & 0 & 0 & 0 & -1 & 0 \\
0 & 0 & 0 & 0 & 0 & 0 & 0 & 0 & 0 & 0 & -1
\end{array}\right)
\end{aligned}
$$

and

$$
\begin{aligned}
& \epsilon_{1}=\left(\begin{array}{ccccccccccc}
\frac{1}{q^{2}} & 0 & 0 & 0 & 0 & 0 & 0 & 0 & 0 & 0 & 0 \\
0 & \frac{1}{q^{2}} & 0 & 0 & 0 & 0 & 0 & 0 & 0 & 0 & 0 \\
0 & 0 & \frac{1}{q^{2}} & 0 & 0 & 0 & 0 & 0 & 0 & 0 & 0 \\
0 & 0 & 0 & \frac{1}{q^{2}} & 0 & 0 & 0 & 0 & 0 & 0 & 0 \\
0 & 0 & 0 & 0 & \frac{1}{q^{2}} & 0 & 0 & 0 & 0 & 0 & 0 \\
0 & 0 & 0 & 0 & 0 & \frac{1}{q^{2}} & 0 & 0 & 0 & 0 & 0 \\
0 & 0 & 0 & 0 & 0 & 0 & \frac{1}{q^{2}} & 0 & 0 & 0 & 0 \\
0 & 0 & 0 & 0 & 0 & 0 & 0 & \frac{1}{q} & 0 & 0 & 0 \\
0 & 0 & 0 & 0 & 0 & 0 & 0 & 0 & \frac{1}{q} & 0 & 0 \\
0 & 0 & 0 & 0 & 0 & 0 & 0 & 0 & 0 & \frac{1}{q} & 0 \\
0 & 0 & 0 & 0 & 0 & 0 & 0 & 0 & 0 & 0 & \frac{1}{q}
\end{array}\right) \\
& \epsilon_{2}=\left(\begin{array}{ccccccccccc}
\frac{1}{q} & 0 & 0 & 0 & 0 & 0 & 0 & 0 & 0 & 0 & 0 \\
\frac{-1+q}{q} & \frac{1}{q} & 0 & 0 & 0 & 0 & 0 & 0 & 0 & 0 & 0 \\
0 & 0 & \frac{1}{q} & 0 & 0 & 0 & 0 & 0 & 0 & 0 & 0 \\
0 & 0 & \frac{-1+q}{q} & \frac{1}{q} & 0 & 0 & 0 & 0 & 0 & 0 & 0 \\
0 & 0 & 0 & 0 & \frac{1}{q} & 0 & 0 & 0 & 0 & 0 & 0 \\
0 & 0 & 0 & 0 & \frac{2(-1+q)}{q} & \frac{1}{q} & 0 & 0 & 0 & 0 & 0 \\
0 & 0 & 0 & 0 & 0 & 0 & \frac{1}{q} & 0 & 0 & 0 & 0 \\
0 & 0 & 0 & 0 & 0 & 0 & 0 & \frac{1}{q^{2}} & 0 & 0 & 0 \\
0 & 0 & 0 & 0 & 0 & 0 & 0 & 0 & \frac{1}{q^{2}} & 0 & 0 \\
0 & 0 & 0 & 0 & 0 & 0 & 0 & 0 & 0 & \frac{1}{q^{2}} & 0 \\
0 & 0 & 0 & 0 & 0 & 0 & 0 & 0 & 0 & 0 & 1
\end{array}\right),
\end{aligned}
$$




$$
\begin{aligned}
& \epsilon_{3}=\left(\begin{array}{ccccccccccc}
\frac{1}{q} & 0 & 0 & 0 & 0 & 0 & 0 & 0 & 0 & 0 & 0 \\
\frac{1-q}{q} & \frac{1}{q} & 0 & 0 & 0 & 0 & 0 & 0 & 0 & 0 & 0 \\
0 & 0 & \frac{1}{q} & 0 & 0 & 0 & 0 & 0 & 0 & 0 & 0 \\
0 & 0 & \frac{1-q}{q} & \frac{1}{q} & 0 & 0 & 0 & 0 & 0 & 0 & 0 \\
0 & 0 & 0 & 0 & 1 & 0 & 0 & 0 & 0 & 0 & 0 \\
0 & 0 & 0 & 0 & 2(-1+q) & 1 & 0 & 0 & 0 & 0 & 0 \\
0 & 0 & 0 & 0 & 0 & 0 & 1 & 0 & 0 & 0 & 0 \\
0 & 0 & 0 & 0 & 0 & 0 & 0 & \frac{1}{q} & 0 & 0 & 0 \\
0 & 0 & 0 & 0 & 0 & 0 & 0 & 0 & \frac{1}{q} & 0 & 0 \\
0 & 0 & 0 & 0 & 0 & 0 & 0 & 0 & 0 & 1 & 0 \\
0 & 0 & 0 & 0 & 0 & 0 & 0 & 0 & 0 & 0 & \frac{1}{q^{2}}
\end{array}\right) \\
& \epsilon_{4}=\left(\begin{array}{ccccccccccc}
1 & 0 & 0 & 0 & 0 & 0 & 0 & 0 & 0 & 0 & 0 \\
0 & 1 & 0 & 0 & 0 & 0 & 0 & 0 & 0 & 0 & 0 \\
2(-1+q) & 0 & 1 & 0 & 0 & 0 & 0 & 0 & 0 & 0 & 0 \\
0 & 2(-1+q) & 0 & 1 & 0 & 0 & 0 & 0 & 0 & 0 & 0 \\
0 & 0 & 0 & 0 & \frac{1}{q} & 0 & 0 & 0 & 0 & 0 & 0 \\
0 & 0 & 0 & 0 & \frac{2(1-q)}{q} & \frac{1}{q} & 0 & 0 & 0 & 0 & 0 \\
0 & 0 & 0 & 0 & 0 & 0 & q & 0 & 0 & 0 & 0 \\
0 & 0 & 0 & 0 & 0 & 0 & 0 & 1 & 0 & 0 & 0 \\
0 & 0 & 0 & 0 & 0 & 0 & 0 & 2(-1+q) & 1 & 0 & 0 \\
0 & 0 & 0 & 0 & 0 & 0 & 0 & 0 & 0 & \frac{1}{q} & 0 \\
0 & 0 & 0 & 0 & 0 & 0 & 0 & 0 & 0 & 0 & \frac{1}{q}
\end{array}\right) .
\end{aligned}
$$

Again, by Reeder's results, these matrices satisfy the necessary relations to give $M$ the structure of an $H_{\mathfrak{B}, \mathcal{O}}$-module. Considering again the exponent $[2,1,1,0]$, which occurs with multiplicity 4 , we deduce that $M \otimes_{\mathcal{O}} K \cong Y_{K}^{\mathfrak{B}}$. We define $Y_{\mathcal{O}}^{\mathfrak{B}}$ to be the image of $M$ under such an isomorphism. We claim that, as in the previous case, $M \otimes_{\mathcal{O}} k$ is an absolutely irreducible $H_{\mathfrak{B}, k}$-module. To this end, let us write $\mathbf{e}_{1}, \ldots, \mathbf{e}_{11}$ for the standard basis of $M \otimes_{\mathcal{O}} k \cong k^{11}$, and let $V \subset M \otimes_{\mathcal{O}} k$ be a simple $H_{\mathfrak{B}, k}$-submodule. We have a decomposition

$$
V=V_{[2,1,1,0]} \oplus V_{[2,1,0,1]} \oplus V_{[2,1,0,-1]} \oplus V_{[1,2,1,0]} \oplus V_{[1,2,0,1]} \oplus V_{[1,0,2,1]}
$$

into generalized $k[X]$-eigenspaces, where

$$
\begin{gathered}
V_{[2,1,1,0]}=V \cap\left\langle\mathbf{e}_{1}, \ldots, \mathbf{e}_{4}\right\rangle, V_{[2,1,0,1]}=V \cap\left\langle\mathbf{e}_{5}, \mathbf{e}_{6}\right\rangle, V_{[2,1,0,-1]}=V \cap\left\langle\mathbf{e}_{7}\right\rangle, \\
V_{[1,2,1,0]}=V \cap\left\langle\mathbf{e}_{8}, \mathbf{e}_{9}\right\rangle, V_{[1,2,0,1]}=V \cap\left\langle\mathbf{e}_{10}\right\rangle, V_{[1,0,2,1]}=V \cap\left\langle\mathbf{e}_{11}\right\rangle .
\end{gathered}
$$

We will show that $V=M \otimes_{\mathcal{O}} k$. Since $V$ is non-zero, one of these generalized eigenspaces is non-zero; we treat each case in turn. If $V_{[2,1,1,0]} \neq 0$, then acting by $k[X]$ shows that $\mathbf{e}_{4} \in V$. The relations

$$
\mathbf{e}_{2}=T_{s_{4}} \mathbf{e}_{4}-q \mathbf{e}_{4}, \mathbf{e}_{3}=T_{s_{2}} \mathbf{e}_{4}-q \mathbf{e}_{4}, \mathbf{e}_{1}=T_{s_{4}} \mathbf{e}_{3}-q \mathbf{e}_{3}
$$

show that $\left\langle\mathbf{e}_{1}, \ldots, \mathbf{e}_{4}\right\rangle \subset V$. The relations

$\mathbf{e}_{6}=(q(q+1))^{-1}\left(T_{s_{3}} \mathbf{e}_{3}-q \mathbf{e}_{4}+\mathbf{e}_{3}\right), \mathbf{e}_{5}=(q(q+1))^{-1}\left(T_{s_{3}} \mathbf{e}_{1}+\mathbf{e}_{1}-q \mathbf{e}_{2}-2 q \mathbf{e}_{3}+2 q(q+1) \mathbf{e}_{4}+2 q\left(1+4 q+q^{2}\right) \mathbf{e}_{6}\right)$ show that $\left\langle\mathbf{e}_{1}, \ldots, \mathbf{e}_{6}\right\rangle \subset V$. The relations

$$
\begin{aligned}
& \mathbf{e}_{7}=(2 q(q+1))^{-1}\left(T_{s_{4}} \mathbf{e}_{5}+\mathbf{e}_{5}-2 q \mathbf{e}_{6}\right), \mathbf{e}_{8}=(q(q+1))^{-1}\left(T_{s_{1}} \mathbf{e}_{1}+\mathbf{e}_{1}-q \mathbf{e}_{2}\right), \\
& \mathbf{e}_{9}=(q(q+1))^{-1}\left(T_{s_{1}} \mathbf{e}_{3}+\mathbf{e}_{3}-q \mathbf{e}_{4}\right), \mathbf{e}_{10}=(2 q(q+1))^{-1}\left(T_{s_{1}} \mathbf{e}_{5}+\mathbf{e}_{5}-2 q \mathbf{e}_{6}\right)
\end{aligned}
$$

show that $\left\langle\mathbf{e}_{1}, \ldots, \mathbf{e}_{10}\right\rangle \subset V$. Finally, the relation

$$
\mathbf{e}_{11}=(q+1)^{2}\left(q\left(q^{2}+q+1\right)\right)^{-1}\left(T_{s_{2}} \mathbf{e}_{10}-(q+1) \mathbf{e}_{10}\right)
$$


shows that in fact $V=M \otimes_{\mathcal{O}} k$. (Note that $q^{2}+q+1 \neq 0$ in $k$, because $q$ is assumed to be a primitive root modulo 7 .)

Now suppose instead that $V_{[2,1,0,1]} \neq 0$. Then $\mathbf{e}_{6} \in V$, hence $\mathbf{e}_{4}=T_{s_{3}} \mathbf{e}_{6}-q \mathbf{e}_{6} \in V$. This shows that $V_{[2,1,1,0]} \neq 0$, hence $V=M \otimes_{\mathcal{O}} k$.

Now suppose instead that $V_{[2,1,0,-1]} \neq 0$, hence $\mathbf{e}_{7} \in V$. The relation $\mathbf{e}_{6}=T_{s_{4}} \mathbf{e}_{7}-q \mathbf{e}_{7}$ shows that $V_{[2,1,0,1]} \neq 0$, hence $V=M \otimes_{\mathcal{O}} k$.

Now suppose instead that $V_{[1,2,1,0]} \neq 0$. If $v \in V_{[1,2,1,0]}$ is non-zero then $T_{s_{1}} v-q v \in V_{[2,1,1,0]}$ is non-zero, hence $V=M \otimes_{\mathcal{O}} k$.

Now suppose instead that $V_{[1,2,0,1]} \neq 0$, hence $\mathbf{e}_{10} \in V$. Then the relation $\mathbf{e}_{9}=T_{s_{3}} \mathbf{e}_{10}-q \mathbf{e}_{10}$ shows that $V_{[1,2,1,0]} \neq 0$, hence $V=M \otimes_{\mathcal{O}} k$.

Now suppose that $V_{[1,0,2,1]} \neq 0$, hence $\mathbf{e}_{11} \in V$. The relation $\mathbf{e}_{10}=T_{s_{2}} \mathbf{e}_{11}-q^{2}(q+1)^{-1} \mathbf{e}_{11}$ shows that $V_{[1,2,0,1]} \neq 0$, hence $V=M \otimes_{\mathcal{O}} k$. In all cases we therefore have $V=M \otimes_{\mathcal{O}} k$, and this concludes the proof that $M \otimes_{\mathcal{O}} k$ is an absolutely irreducible $H_{\mathfrak{B}, k}$-module.

One can construct an integral structure in $Z_{K}^{\mathfrak{B}}$ in the same way as for $Y_{K}^{\mathfrak{B}}$ above; alternatively, it is

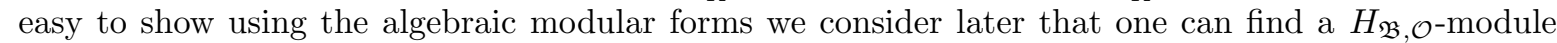
$N$ which is finite free as an $\mathcal{O}$-module and such that there exists an injection $Z_{K}^{\mathfrak{B}} \hookrightarrow N \otimes_{\mathcal{O}} K$ of $H_{\mathfrak{B}, K}$-modules. One can then define $Z_{\mathcal{O}}^{\mathfrak{B}}=N \cap Z_{K}^{\mathfrak{B}}$. Since the exponents of the Jacquet module of $Z_{K}$ are distinct, modulo $\lambda$, from the exponent of $X_{K}$, the assertion about Jordan-Hölder factors now follows from the assertion that $Y_{\mathcal{O}}^{\mathfrak{B}} \otimes_{\mathcal{O}} k$ is an absolutely irreducible $H_{\mathfrak{B}, k}$-module. This completes the proof of the proposition.

Remark 2.7. The matrices described above can be used to define the module $Y_{K}^{\mathfrak{B}}$ without restriction on the order of $q$ modulo $l$. We use the hypothesis that $q$ is a primitive root modulo $l$ to ensure that they determine an integral structure $Y_{\mathcal{O}}^{\mathfrak{B}} \subset Y_{K}^{\mathfrak{B}}$. This observation will be used in the proof of Proposition 2.9 below.

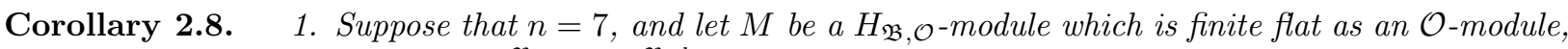
and such that $M \otimes_{\mathcal{O}} K \cong\left(X_{K}^{\mathfrak{B}}\right)^{a} \oplus\left(Y_{K}^{\mathfrak{B}}\right)^{b}$ as $H_{\mathfrak{B}, K}$-modules, for some integers $a, b \geq 0$. Let $M_{X} \subset M$ denote the intersection of $M$ with the $X_{K}^{\mathfrak{B}}$-isotypic part of $M \otimes_{\mathcal{O}} K$, and define $M_{Y}$ similarly.

Suppose that $q$ is a primitive root modulo l. Then the natural map $M_{X} \oplus M_{Y} \rightarrow M$ is an isomorphism.

2. Suppose that $n=9$, and let $M$ be a $H_{\mathfrak{B}, \mathcal{O}}$-module which is finite flat as an $\mathcal{O}$-module, and such that $M \otimes_{\mathcal{O}} K \cong\left(X_{K}^{\mathfrak{B}}\right)^{a} \oplus\left(Y_{K}^{\mathfrak{B}}\right)^{b} \oplus\left(Z_{K}^{\mathfrak{B}}\right)^{c}$ as $H_{\mathfrak{B}, K}$-modules, for some integers $a, b, c \geq 0$. Let $M_{X} \subset M$ denote the intersection of $M$ with the $X_{K}^{\mathfrak{B}}$-isotypic part of $M \otimes_{\mathcal{O}} K$, and define $M_{Y}, M_{Z}$ similarly.

Suppose that $q$ is a primitive root modulo $l$. Then the natural map $M_{X} \oplus M_{Y} \oplus M_{Z} \rightarrow M$ is an isomorphism.

Proof. We prove the second part; see [CT15, Corollary 2.10] for the first part. The map $M_{X} \oplus M_{Y} \oplus$ $M_{Z} \rightarrow M$ becomes an isomorphism after inverting $l$, so to prove the result it suffices to show that the map $\left(M_{X} \oplus M_{Y} \oplus M_{Z}\right) \otimes_{\mathcal{O}} k \rightarrow M \otimes_{\mathcal{O}} k$ is injective. Let $L$ be a simple submodule of the kernel. Being non-zero, the projection of $L$ to at least one of $M_{X} \otimes_{\mathcal{O}} k, M_{Y} \otimes_{\mathcal{O}} k$, and $M_{Z} \otimes_{\mathcal{O}} k$ must be non-zero. However, the maps

$$
M_{X} \otimes_{\mathcal{O}} k \rightarrow M \otimes_{\mathcal{O}} k, M_{Y} \otimes_{\mathcal{O}} k \rightarrow M \otimes_{\mathcal{O}} k, \text { and } M_{Z} \otimes_{\mathcal{O}} k \rightarrow M \otimes_{\mathcal{O}} k
$$

are injective by definition. It follows that the projection of $L$ to at least two of $M_{X} \otimes_{\mathcal{O}} k, M_{Y} \otimes_{\mathcal{O}} k$, and $M_{Z} \otimes_{\mathcal{O}} k$ must be non-zero. However, this implies that these two spaces have a simple submodule in common, contradicting Proposition 2.6. This completes the proof.

According to the Bernstein presentation, the algebra $H_{\mathfrak{B}, \mathcal{O}}$ has an abelian subalgebras $\mathcal{O}[\Lambda]$. We let $\chi: \mathcal{O}[\Lambda] \rightarrow \mathcal{O}$ denote the character giving the action of $\mathcal{O}[\Lambda]$ on $X_{K}^{\mathfrak{B}}$, and let $\bar{\chi}: \mathcal{O}[\Lambda] \rightarrow k$ denote its reduction modulo $\lambda$. If $M$ is a $\mathcal{O}[\Lambda]$-module, then we write $M(\bar{\chi})$ for its localization at ker $\bar{\chi}$. We recall that the algebra $H_{\mathfrak{B}, \mathcal{O}}$ has a canonical anti-involution $\jmath$ given on double cosets by $[\mathfrak{B} g \mathfrak{B}] \mapsto\left[\mathfrak{B} g^{-1} \mathfrak{B}\right]$. Thus 
there is another abelian subalgebra $j \mathcal{O}[\Lambda] \subset H_{\mathfrak{B}, \mathcal{O}}$, with a character $\bar{\chi} \jmath=\bar{\chi} \circ \jmath$, and we write $M(\bar{\chi} \jmath)$ for the analogous localization.

Proposition 2.9. Suppose that $q \equiv-1 \bmod l$. Then:

1. Suppose that $n=7$. Then $X_{K}^{\mathfrak{B}}=X_{K}^{\mathfrak{B}}(\bar{\chi})=X_{K}^{\mathfrak{B}}(\bar{\chi} \jmath)$ and $\operatorname{dim}_{K} Y_{K}^{\mathfrak{P}} \cap Y_{K}^{\mathfrak{B}}(\bar{\chi})=\operatorname{dim}_{K} Y_{K}^{\mathfrak{P}} \cap Y_{K}^{\mathfrak{B}}(\bar{\chi} \jmath)=1$.

2. Suppose that $n=9$. Then $X_{K}^{\mathfrak{B}}=X_{K}^{\mathfrak{B}}(\bar{\chi})=X_{K}^{\mathfrak{B}}(\bar{\chi} \jmath), \operatorname{dim}_{K} Y_{K}^{\mathfrak{P}} \cap Y_{K}^{\mathfrak{B}}(\bar{\chi})=\operatorname{dim}_{K} Y_{K}^{\mathfrak{P}} \cap Y_{K}^{\mathfrak{B}}(\bar{\chi} \jmath)=1$ and $Z_{K}^{\mathfrak{B}}(\bar{\chi})=0$.

Proof. The assertion that $X_{K}^{\mathfrak{B}}=X_{K}^{\mathfrak{B}}(\bar{\chi})=X_{K}^{\mathfrak{B}}(\bar{\chi} \jmath)$ follows from the fact that $X_{K}$ is self-dual (as follows easily from Theorem 3.3) and $X_{K}^{\mathfrak{B}}$ is 1-dimensional, cf. [CT15, Proposition 2.8]. The assertion in the second case that $Z_{K}^{\mathfrak{B}}(\bar{\chi})=0$ follows from the earlier calculation of Jacquet modules. The assertion that $\operatorname{dim}_{K} Y_{K}^{\mathfrak{P}} \cap Y_{K}^{\mathfrak{B}}(\bar{\chi})=1$ follows from the observation that if $V$ is an admissible $\mathbb{C}\left[U_{n}\right]$-module, $v \in V^{\mathfrak{B}}$ and $T_{s_{k}} v=q v$, then $v \in V^{\mathfrak{P}}$, together with the above explicit matrices. (Observe that the operator $e_{\mathfrak{P}}=[\mathfrak{P}] /(q+1)=\left([\mathfrak{B}]+\left[\mathfrak{B} s_{k} \mathfrak{B}\right]\right) /(q+1)$ is a projector onto the set of $\mathfrak{P}$-fixed vectors. $)$

To show that $\operatorname{dim}_{K} Y_{K}^{\mathfrak{P}} \cap Y_{K}^{\mathfrak{B}}(\bar{\chi} \jmath)=1$, we give another characterization of this subspace. The representation $Y_{K}$ is self-dual (again, by Theorem 3.3), from which it follows that there exists a perfect duality $\langle\cdot, \cdot\rangle: Y_{K}^{\mathfrak{B}} \times Y_{K}^{\mathfrak{B}} \rightarrow K$ satisfying $\langle t x, y\rangle=\langle x, \jmath(t) y\rangle$ for all $t \in H_{\mathfrak{B}, \mathcal{O}}, x, y \in Y_{K}^{\mathfrak{B}}$.

The space $Y_{K}^{\mathfrak{B}}(\bar{\chi} \jmath)$ can be characterized as the annihilator of the spaces $Y_{K}^{\mathfrak{B}}(\bar{\eta}), \bar{\eta} \neq \bar{\chi}$, under the above duality. The assertion that $Y_{K}^{\mathfrak{P}} \cap Y_{K}^{\mathfrak{B}}(\bar{\chi} \jmath)$ is of dimension 1 is therefore equivalent to the assertion that the span of the spaces $Y_{K}^{\mathfrak{B}}(\bar{\eta}), \bar{\eta} \neq \bar{\chi}$, together with the annihilator of $Y_{K}^{\mathfrak{P}}=e_{\mathfrak{P}} Y_{K}^{\mathfrak{B}}$, is of codimension 1 in $Y_{K}^{\mathfrak{B}}$. Since $\jmath\left(e_{\mathfrak{P}}\right)=e_{\mathfrak{P}}$, this is itself equivalent to the assertion that the subspace of $Y_{K}^{\mathfrak{B}}$ spanned by the subspaces $Y_{K}^{\mathfrak{B}}(\bar{\eta}), \bar{\eta} \neq \bar{\chi}$, together with $\left(1-e_{\mathfrak{P}}\right) Y_{K}^{\mathfrak{B}}$, is of codimension 1 . This can again be readily checked using the matrices appearing in the proof of Proposition 2.6.

\section{Unitary groups and transfer}

Recall from the introduction (in the special case of $\mathrm{Sym}^{8}$ ) the identity (1.1):

$$
\operatorname{Sym}^{8} \bar{r} \cong\left({ }^{\varphi} \bar{r} \otimes \bar{r}\right) \oplus \bar{\chi}^{2} \operatorname{Sym}^{4} \bar{r},
$$

true for (semi-simplified) representations of $\mathrm{GL}_{2}\left(\overline{\mathbb{F}}_{7}\right)$, and therefore for a Galois representation $\bar{r}$ of degree $2(\bmod 7)$. We may, for simplicity, assume that $\bar{r}$ has large image, so the two summands are irreducible. If $\bar{r}$ arises from a RAESDC automorphic representation of $\mathrm{GL}_{2}\left(\mathbb{A}_{F}\right), F$ a totally real field, then the two summands are themselves known to arise from RAESDC representations of $\mathrm{GL}_{4}\left(\mathbb{A}_{F}\right)$ and $\mathrm{GL}_{5}\left(\mathbb{A}_{F}\right)$ (these are known to exist, by the results of Ramakrishnan and Kim-Shahidi).

Starting with these representations $\Pi_{4}, \Pi_{5}$, we will construct in this section a "packet" (in the sense of Arthur and Langlands) of representations of a unitary group $G$ in 9 variables over the totally real field $F$; $G$ will be compact at the Archimedean places (and quasi-split elsewhere).

If we replace $G$ by its quasi-split form $G^{*}$, we are reduced to the construction by Mok of endoscopic L-packets (Theorem 3.5). At the local primes of $F$ ramified in the (CM) extension $E$ defining $G$, we obtain, for suitable carefully chosen initial data $\Pi_{4}, \Pi_{5}$, the representations described in $\S 2$. In order to understand these representations, we complete in this section the proof of Theorem 2.3, and state a more precise result (see Theorem 3.2 and the following paragraph) describing the Arthur-Mok signs of the representations in the local L-packets. This requires delicate local computations $(\S \S 3.4-3.5)$.

We are then ready to transfer Mok's representations to the $\mathbb{R}$-anistropic group $G$. This is done in $\S 3.6$, using the methods in [CHL11]. The results are stated in Theorem 3.8, Theorem 3.10. It is again crucial that, at the primes ramified in $E / F$, the local components are controlled by the sign formula.

We should mention that the representation $\Pi_{4}$ directly obtained from our given representation of $\mathrm{GL}_{2}$ is not directly useful, as $\Pi_{4} \boxplus \Pi_{5}$ does not yield a cohomological representation of $G^{*}$, and therefore does not transfer to $G$. This is easily obviated by a standard weight-changing argument (see [CT15, Proposition $3.10])$ which is reviewed in $\S 6$. 


\subsection{Unitary groups}

Let $E / F$ be a quadratic extension of local or global fields. We consider a unitary group $G$ in $n$ variables associated to this extension. Thus

$$
{ }^{L} G=\mathrm{GL}_{n}(\mathbb{C}) \rtimes W_{F}
$$

where $W_{F}$ acts through $\operatorname{Gal}(E / F)$, the generator $c$ of $\operatorname{Gal}(E / F)$ acting by

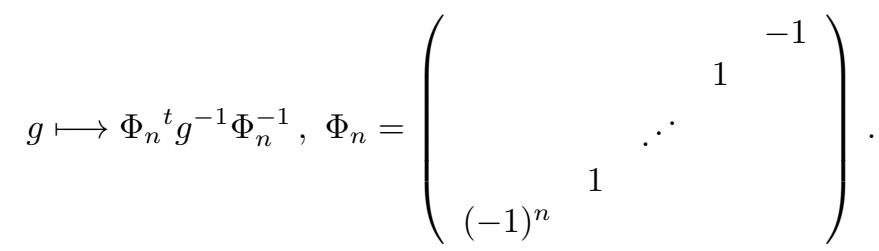

In our case, $n\left(=l+2, l\right.$ an odd prime) will be odd. Over $E, G$ becomes isomorphic to $\mathrm{GL}_{n}$ and there is an L-homomorphism

$$
{ }^{L} G \longrightarrow{ }^{L}\left(\operatorname{Res}_{E / F} G_{E}\right)
$$

of L-groups over $F$, given by

$$
(g, w) \longmapsto(g, g, w)\left(w \in W_{F}\right),
$$

$W_{F}$ acting on $\left(\operatorname{Res}_{E / F} G_{E}\right)^{\wedge}=\mathrm{GL}_{n}(\mathbb{C}) \times \mathrm{GL}_{n}(\mathbb{C})$ through $\operatorname{Gal}(E / F), c$ acting by

$$
(g, h) \longmapsto\left(\Phi_{n}^{t} h^{-1} \Phi_{n}^{-1}, \Phi_{n}^{t} g^{-1} \Phi_{n}^{-1}\right) .
$$

If $F$ is local, we write $\Phi_{\text {bdd }}(G)$ for the set of equivalence classes of parameters $L_{F} \rightarrow{ }^{L} G$ with bounded restriction to $W_{F}$ (cf. [CT15, 22.2$]$ ), where

$$
L_{F}=\left\{\begin{array}{cc}
W_{F} & F \text { Archimedean } \\
W_{F} \times \mathrm{SU}_{2}(\mathbb{R}) & F p \text {-adic. }
\end{array}\right.
$$

By composing with ${ }^{L} G \rightarrow{ }^{L}\left(\operatorname{Res}_{E / F} G\right)$ we obtain parameters for $\operatorname{GL}_{n}(E)$. Then, $n$ being odd, we have (see $\S 2.2$ and [Mok15, Lemma 2.2.1]):

Lemma 3.1. This map $\left.\varphi \mapsto \varphi\right|_{L_{E}}$ induces a bijection between $\Phi_{b d d}(G)$ and the set of parameters in $\Phi_{b d d}\left(\mathrm{GL}_{n}(E)\right)$ that are conjugate orthogonal.

We refer to this map of parameters as the stable base change map. If $\mu: E^{\times} \rightarrow \mathbb{C}^{\times}$is a continuous character such that $\left.\mu\right|_{F^{\times}}$is the character of order two $\varepsilon_{E / F}$ given by class field theory, then the map $\left.\varphi \mapsto \varphi\right|_{L_{E}} \otimes \mu$ defines a bijection between $\Phi_{\mathrm{bdd}}(G)$ and the set of parameters in $\Phi_{\mathrm{bdd}}\left(\mathrm{GL}_{n}(E)\right)$ that are conjugate symplectic (see [Mok15, Lemma 2.2.1]). In particular, the image of this map is independent of the choice of $\mu$, and we refer to this map on parameters as the unstable base change map.

We now return to the general case ( $F$ local or global) and consider the endoscopic group $H$ for $G$, isomorphic to $U(n-4) \times U(4)$, the unitary groups being quasi-split. The group ${ }^{L} H$ admits the same description, with $\widehat{H}=\mathrm{GL}_{m}(\mathbb{C}) \times \mathrm{GL}_{4}(\mathbb{C})$ and the matrices $\Phi_{m}, \Phi_{4}$. (We set $m=n-4$.) There is an embedding $\xi:{ }^{L} H \longrightarrow{ }^{L} G$ given by the following formulas. We fix a character $\mu$ of $E^{\times}$(local case), or of $E^{\times} \backslash \mathbb{A}_{E}^{\times}$(global case) whose restriction to $F^{\times}$(resp. $\mathbb{A}_{F}^{\times}$) is the character of order two $\varepsilon_{E / F}$ given by class field theory. Then [Mín11, p. 404] $\xi$ is given by

$$
\begin{aligned}
\left(g_{1}, g_{2}\right) \times 1 & \longmapsto\left(\begin{array}{cc}
g_{1} & \\
& g_{2}
\end{array}\right),\left(g_{1}, g_{2}\right) \in \widehat{H} \\
1 \times w & \longmapsto\left(\left(\begin{array}{cc}
1_{m} & \\
& \mu(w) 1_{4}
\end{array}\right), w\right), w \in W_{E} \\
1 \times w_{c} & \longmapsto\left(\left(\begin{array}{cc}
\Phi_{m} & \\
& \Phi_{4}
\end{array}\right) \Phi_{n}^{-1}, w_{c}\right)
\end{aligned}
$$

where $w_{c} \in W_{F}-W_{E}$ is a representative of $c$. 
For some computations it is expedient to replace $\xi$ by the following, conjugate to the previous one by an element of $\widehat{G}$. Write

$$
g_{2}=\left(\begin{array}{cc}
A & B \\
C & D
\end{array}\right)
$$

(blocks of size 2), and consider

$$
\begin{aligned}
\xi:\left(g_{1}, g_{2}\right) & \mapsto\left(\begin{array}{ccc}
A & & B \\
& g_{1} & \\
C & & D
\end{array}\right) \\
1 \times w & \mapsto\left(\left(\begin{array}{ccc}
\mu(w) 1_{2} & & \\
& 1_{m} & \\
& & \mu(w) 1_{2}
\end{array}\right), w\right), w \in W_{E} \\
1 \times w_{c} & \mapsto\left(\left(\begin{array}{lll} 
& & \Phi_{2} \\
& \Phi_{m} & \\
\Phi_{2} & &
\end{array}\right) \Phi_{n}^{-1}, w_{c}\right.
\end{aligned}
$$

\subsection{Local L-packets}

Assume now that $E / F$ is an extension of $p$-adic fields. There is a conjugate orthogonal parameter $\varphi_{E} \in$ $\Phi_{\text {bdd }}\left(\mathrm{GL}_{n}(E)\right)$ associated to the induced representation

$$
\Pi_{E}=\mathrm{St}_{m, E} \boxplus \mathrm{St}_{3, E} \boxplus \mathrm{St}_{1, E}
$$

If $l>5$, thus $m>3$, this is a discrete parameter in the sense of Møglin [Mœg07], thus associated to a packet of discrete series for $G$. If $l=5, \Pi_{E}$ is associated to the reducible representation of $G$ :

$$
\pi=\mathrm{n}-\operatorname{Ind}_{P}^{G}\left(\operatorname{St}_{3, E} \otimes \mathbf{1}\right)
$$

where $P$ is the parabolic subgroup of $G$ with Levi subgroup $\mathrm{GL}_{3}(E) \times U(1)$.

Theorem 3.2 (Mœglin, Mok). The parameter $\varphi_{E}$ determines an L-packet $\Pi_{\varphi}$ of tempered representations of $G$.

(i) If $m=3, \Pi_{\varphi}$ has two elements $X, Y$.

(ii) If $m \geq 5, \Pi_{\varphi}$ has four elements $X, Y, Z, W$ which belong to the discrete series of $G$.

This follows from Arthur's formalism; we refer to [Mok15, Theorem 2.5.1]. Let $\varphi: L_{F} \rightarrow{ }^{L} G$ be the parameter deduced from $\varphi_{E}$. We must compute

$$
\begin{aligned}
& S_{\varphi}=\operatorname{Cent}(\operatorname{Im} \varphi, \widehat{G}), \\
& \mathcal{S}_{\varphi}=\pi_{0}\left(S_{\varphi} /\{ \pm 1\}\right) .
\end{aligned}
$$

(See [GGP12, §4] for the general calculation.) In case (i) $S_{\varphi}=\mathrm{O}_{2}(\mathbb{C}) \times\{ \pm 1\}, \mathcal{S}_{\varphi}=\{ \pm 1\}$. If we assume $\varphi_{E}$ written as $s p(3) \oplus s p(1) \oplus s p(3), s p(r)$ denoting the irreducible representation of degree $r$ of $\mathrm{SU}_{2}(\mathbb{R})$, the non-trivial element of $\mathcal{S}_{\varphi}$ is represented by

$$
\left(\begin{array}{lll} 
& & 1_{3} \\
& 1 & \\
1_{3} & &
\end{array}\right)
$$

(block scalar matrices).

In case (ii) $\mathcal{S}_{\varphi}$ is represented by

$$
\left(\begin{array}{lll}
a & & \\
& b & \\
& & c
\end{array}\right)
$$


$(a, b, c= \pm 1) \bmod \{ \pm 1\}$, the sizes being $(1,3, m)$. We recall that there is a non-degenerate pairing of $\Pi_{\varphi}$ with $\mathcal{S}_{\phi}$. In case (i) we set $\varepsilon(X)=-1$ and $\varepsilon(Y)=1, \varepsilon$ being the unique non-trivial character. In case (ii) we set, $\varepsilon_{1}, \varepsilon_{2}$ being associated with the components $a, b$ :

\begin{tabular}{c|cccc}
$\pi$ & $X$ & $Y$ & $Z$ & $W$ \\
\hline$\varepsilon_{1}(\pi)$ & 1 & 1 & -1 & -1 \\
$\varepsilon_{2}(\pi)$ & -1 & 1 & -1 & 1
\end{tabular}

For the $\operatorname{sign} \varepsilon=\varepsilon_{1} \varepsilon_{2}$, we therefore have $\varepsilon(Y)=\varepsilon(Z)=1$ and $\varepsilon(X)=\varepsilon(W)=-1$.

We will need information on the Jacquet modules of the components of $\Pi\left(\varphi_{E}\right)$. Recall that an irreducible semi-stable representation is, by definition, a subquotient of an unramified principal series, i.e., a principal series induced from a character of the maximally split torus trivial on its maximal compact subgroup. Let $P_{0}=M_{0} N_{0}$ be the Borel subgroup, with Levi subgroup $\left(E^{\times}\right)^{k} \times U(1)$. (Thus $k=(m+3) / 2$.) Let us denote by $e=\left[e_{1}, \ldots, e_{k}\right]$ the character of $\left(E^{\times}\right)^{k} \times U(1)$

$$
\left(z_{1}, \ldots z_{k}, u\right) \longmapsto\left|z_{1}\right|^{e_{1}} \cdots\left|z_{k}\right|^{e_{k}} . \quad\left(z_{i} \in E^{\times}, u \in U(1)\right) .
$$

For any semi-stable representation $\pi$, the normalized Jacquet module $\pi_{N_{0}}^{\text {norm }}$ is, after semi-simplification, a sum of characters ("exponents") $e$, with multiplicities.

Theorem 3.3. Suppose that $E / F$ is ramified, and that the residue characteristic of $F$ is odd. Then:

(i) If $m=3, X, Y$ are semi-stable with exponents $[1,0,1]$ and $4[1,1,0]+2[1,0,1]+[1,0,-1]$, respectively.

(ii) If $m=5, X, Y, Z$ are semi-stable with exponents as follows:

$$
\begin{aligned}
& X:[2,1,0,-1] \\
& Y: \quad[2,1,0,-1], 2[2,1,0,1], 4[2,1,1,0],[1,0,2,1],[1,2,0,1], 2[1,2,1,0] \\
& Z \quad: \quad[1,0,2,1],[1,2,0,1] \text {. }
\end{aligned}
$$

(iii) For $m>5, X, Y, Z$ are semi-stable and their exponents may be described inductively as follows, $k$ being equal to $\frac{n-1}{2}=\frac{m+3}{2}$. Write $X_{k}, Y_{k}, Z_{k}$. Then $\left(X_{k}\right)_{N_{0}}^{\text {norm }}$ is 1-dimensional of exponent $[k-2, k-$ $3, \ldots 0,-1]$. The exponents of $Y_{k}$ and $Z_{k}$ comprise the exponents $\left[k-2, e\left(Y_{k-1}\right)\right],\left[k-2, e\left(Z_{k-1}\right)\right]$ for all exponents of $Y_{k-1}, Z_{k-1}$, with the same multiplicities, together with:

$$
\begin{aligned}
& Y_{k}:[1,0, k-2, \ldots, 1],[1, k-2,0, k-3, \ldots, 1], \ldots,[1, k-2, k-3, \ldots, 0,1], 2[1, k-2, \ldots, 1,0] \\
& Z_{k}:[1,0, \ldots, k-2, \ldots, 1],[1, k-2,0, k-3, \ldots 1], \ldots,[1, k-2, \ldots, 0,1] \text {. }
\end{aligned}
$$

The proof of Theorem 3.3 will be given in $\S 3.5$ below.

\subsection{Global L-packets}

We now describe the global analogue of this construction, only so far in the quasi-split case. We now assume $E / F$ is a totally imaginary quadratic extension of a totally real field. Choose, for each Archimedean prime $v$ of $F$, a prime $w$ of $E$ above $F$ so $E_{w}=\mathbb{C}$. If $a$ is a positive integer, then we will write $U(a)$ for the quasi-split unitary group over $F$ in $a$ variables, split by $E$.

Lemma 3.4. There exists a continuous homomorphism $\mu: E^{\times} \backslash \mathbb{A}_{E}^{\times} \rightarrow \mathbb{C}^{\times}$such that $\left.\mu\right|_{\mathbb{A}_{F}^{\times}}=\varepsilon_{E / F}$ and, for each Archimedean prime $w$ and $z \in E_{w} \cong \mathbb{C}, \mu(z)=(z / \bar{z})^{1 / 2}$.

Proof. Let $E_{\infty}=\prod_{w \mid \infty} E_{w} \cong \prod_{w} \mathbb{C}$, and let $U \subset \mathbb{A}_{E}^{\infty, \times}$ be an open subgroup satisfying the following conditions:

- We have $U \cap E^{\times} \subset \mathcal{O}_{F}^{\times,+}$(the subgroup of totally positive units of $F$ ). 
- We have $\left.\varepsilon_{E / F}\right|_{U \cap \mathbb{A}_{F}^{\infty, x}}=1$.

- The group $U$ is stable under the action of $c \in \operatorname{Gal}(E / F)$.

Then $E^{\times} \cdot \mathbb{A}_{F}^{\times} \cdot\left(U \times E_{\infty}^{\times}\right)$is an open subgroup of $\mathbb{A}_{E}^{\times}$, and $E^{\times} \cap\left(\mathbb{A}_{F}^{\times} \cdot\left(U \times E_{\infty}^{\times}\right)\right) \subset F^{\times}$. Indeed, if $e \in E^{\times}$ satisfies $e=\alpha \cdot\left(u,\left(z_{w}\right)_{w \mid \infty}\right)$ for $\alpha \in \mathbb{A}_{F}^{\times}, u \in U$, and $z_{w} \in E_{w}^{\times}$, then $f=e / e^{c}=\left(u / u^{c},\left(z_{w} / \bar{z}_{w}\right)_{w \mid \infty}\right)$ lies in $E^{\times} \cap U \subset \mathcal{O}_{F}^{\times,+}$. We thus have $f=f^{c}=f^{-1}$, hence $f^{2}=1$, hence $f=1$ (as $f$ is totally positive).

We can therefore define a homomorphism $\mu: E^{\times} \cdot \mathbb{A}_{F}^{\times} \cdot\left(U \times E_{\infty}^{\times}\right) \rightarrow \mathbb{C}^{\times}$by the formula $e \cdot \alpha \cdot$ $\left(u,\left(z_{w}\right)_{w \mid \infty}\right) \mapsto \varepsilon_{E / F}(\alpha) \prod_{w}\left(z_{w} / \bar{z}_{w}\right)^{1 / 2}$. This character is continuous because it is continuous on restriction to the open subgroup $U \times E_{\infty}^{\times}$. The lemma is completed on choosing any extension of $\mu$ to a homomorphism $\mathbb{A}_{E}^{\times} \rightarrow \mathbb{C}^{\times}$

We will consider endoscopic representations $\pi$ of $G(\mathbb{A})$ associated to conjugate self-dual cuspidal representations $\Pi_{m}, \Pi_{4}$ of $\mathrm{GL}_{m}\left(\mathbb{A}_{E}\right), \mathrm{GL}_{4}\left(\mathbb{A}_{E}\right)$. We assume first that

$$
\Pi_{m}^{\vee} \cong \Pi_{m}^{c},
$$

and that for each Archimedean prime $w$, the Langlands parameter of $\Pi_{m}$ is given by

$$
z \longmapsto \operatorname{diag}\left((z / \bar{z})^{\frac{m-1}{2}},(z / \bar{z})^{\frac{m-3}{2}}, \ldots,(z / \bar{z})^{\frac{1-m}{2}}\right) .
$$

Note that this parameter comes, by stable base change, from a parameter for $U(m)$, at least at the Archimedean primes (cf. Lemma 3.1).

For the other representation we assume that

$$
\Pi_{4}^{\vee} \cong \Pi_{4}^{c}
$$

and that the Langlands parameter is given, at each Archimedean prime $w$, by

$$
z \longmapsto \operatorname{diag}\left((z / \bar{z})^{\frac{n-2}{2}},(z / \bar{z})^{\frac{n-4}{2}},(z / \bar{z})^{\frac{2-n}{2}},(z / \bar{z})^{-\frac{n}{2}}\right) .
$$

Again, the representation $\Pi_{4}$ originates from stable base change since $n-2$ is odd. The representation $\Pi_{4} \otimes \mu$ is then still conjugate self-dual, and originates from unstable base change. Its Archimedean parameters are

$$
z \longmapsto \operatorname{diag}\left((z / \bar{z})^{\frac{n-1}{2}},(z / \bar{z})^{\frac{n-3}{2}},(z / \bar{z})^{\frac{3-n}{2}},(z / \bar{z})^{\frac{1-n}{2}}\right) .
$$

We can consider the datum $\left(\Pi_{m}, \Pi_{4} \otimes \mu\right)$ as an Arthur datum $\psi$ in the sense of [Art], [Mok15], for the unitary group $G$. It defines a global group $\mathcal{S}_{\psi}$ [Mok15, Definition 2.4.8], isomorphic to $\{ \pm 1\}$ seen as before as the quotient of the diagonal-scalar matrices $(a, b)(a, b= \pm 1)$ of size $(m, 4)$ by $Z(\widehat{G})^{W_{F}}=\{ \pm 1\}$. We write $s \in \mathcal{S}_{\psi}$ for the non-trivial element.

For each place $v$ of $F, \psi$ determines a local (tempered) parameter $\varphi_{v}: W_{F} \times \mathrm{SU}_{2}(\mathbb{R}) \longrightarrow{ }^{L} G$. If $\pi_{v} \in \Pi_{\varphi_{v}}$ the pairing $\left\langle s, \pi_{v}\right\rangle$ is well-defined. We now have:

Theorem 3.5 (Mok). Assume given, for each $v$, a representation $\pi_{v} \in \Pi_{\varphi_{v}}$, with $\pi_{v}$ almost everywhere unramified. Then the representation $\pi=\otimes_{v} \pi_{v}$ occurs in $L_{\text {cusp }}^{2}(G(F) \backslash G(\mathbb{A}))$ if and only if $\prod_{v}\left\langle s, \pi_{v}\right\rangle=1$. In this case, it occurs with multiplicity one.

This is essentially [Mok15, Theorem 2.5.2], taking into account the fact that the sign $\varepsilon_{\psi}$ is 1 since the parameter $\psi$ is tempered. We have added the fact that $\pi$ is cuspidal in our case: this follows from Mok's proof, implying that $\pi_{v}$ is associated by unramified base change, at almost all primes, with the induced representation $\Pi_{m} \boxplus \Pi_{4} \otimes \mu$. In particular it is tempered; however, a residual representation is tempered at no place [Clo93, Proposition 4.10]. In the next sections, we will prove the analogue of this theorem when $G$ is compact at the Archimedean primes. 


\subsection{Transfer factors}

In this section we prove lemmas needed to transfer the representation $\Pi_{m} \boxplus\left(\Pi_{4} \otimes \mu\right)$ of $\S 3.3$ to a compact unitary group. We will also obtain the local information necessary to prove Theorem 3.3.

Since $n$ is odd, there exists a unitary group $G$, associated to a Hermitian from on $E^{n}$, such that $G\left(F_{v}\right)$ is compact for each Archimedean prime and quasi-split at finite primes. We now denote by $G^{*}$ the quasi-split inner form of $G$. The other elliptic endoscopic groups are of the form $U(a) \times U(b)(n=a+b)$; we are essentially concerned with $H=U(m) \times U(4)$. We follow the arguments of [CT15, §3.4-3.8] to which we will refer when convenient.

We consider a decomposed, smooth, $K_{\infty}$-finite function $f=\otimes_{v} f_{v}$ on $G\left(\mathbb{A}_{F}\right)$. There are associated functions $f^{H}$ on $H\left(\mathbb{A}_{F}\right)$ where $H$ is any endoscopic group. At finite primes, $f_{v} \rightsquigarrow f_{v}^{H}$ is given by the Whittaker normalization of transfer factors. (Note that since we are considering unitary groups of odd absolute rank, there is a unique equivalence class of Whittaker data.) As in [CT15, §3.5] one checks that they are simply the Langlands-Shelstad transfer factors. At the Archimedean primes we have to compare Kottwitz's transfer factor used in [Clo11] and the Langlands-Shelstad factor $\Delta_{0}$. At each real prime we find, as in $[\mathrm{CT} 15, \S 3.5]$, for our particular $H$ :

$$
\Delta_{K}^{G^{*}}(\gamma, \delta)= \pm \Delta_{0}(\gamma, \delta)
$$

$\left(\gamma \in H\left(F_{v}\right), \delta \in G\left(F_{v}\right)\right)$, the sign depending only on the real groups which are the same at all Archimedean places, and

$$
\Delta_{K}^{G^{*}}(\gamma, \gamma)=\Delta_{K}^{G}(\gamma, \gamma)
$$

If $[F: \mathbb{Q}]$ is even, we conclude that we can use Kottwitz's factors at infinity and the LanglandsShelstad factors at finite primes.

We must next understand the spectral transfer factors, first at the real primes [CT15, §3.6]. Since we are using Kottwitz's transfer factors we can use the analysis in [Clo11]. Let $\Theta_{\varphi_{v}, H}$ be the (stable) character of the $\mathrm{L}$-packet for $H(\mathbb{R})$ associated to the representation of $H(\mathbb{C})=\mathrm{GL}_{m}(\mathbb{C}) \times \mathrm{GL}_{4}(\mathbb{C})$ that is the local component of $\left(\Pi_{m}, \Pi_{4} \otimes \mu\right)$ at a place $w \mid v$. Let $\mathbb{C}$ denote the trivial representation of $G(\mathbb{R})$. Then, the transfer $f_{v} \rightsquigarrow f_{v}^{H}$ being defined by Kottwitz's factor,

$$
\left\langle\Theta_{\varphi_{v}, H}, f_{v}^{H}\right\rangle=\operatorname{det}(w)\left\langle\Theta_{\mathbb{C}}, f_{v}\right\rangle
$$

where $w$ is described in [Clo11, Theorem 3.4]. (This is due to Kottwitz and Shelstad.) In our case $\operatorname{det}(w)=1$. Also note that the cocyle $a_{w \sigma}$ there is equal to 1 since $G\left(F_{v}\right)$ is compact.

We now come to the geometric transfer factors at the $p$-adic places. They are the Langlands-Shelstad factors, but we will need explicit formulas for them on (associated) maximally split tori in $G=G\left(F_{v}\right)$ and $H=H\left(F_{v}\right)$. We could use the formulas in [Wal10], but we hope this exposition will clarify the relation with our parameters. We have $T_{G} \cong\left(E^{\times}\right)^{k} \times U(1)$, and

$$
T_{H} \cong\left(\left(E^{\times}\right)^{k-2} \times U(1)\right) \times\left(E^{\times}\right)^{2} \subset U(m) \times U(4) .
$$

Elements of $T_{G}, T_{H}$ can be conjugated by the obvious isomorphisms in $\mathrm{GL}_{n}(E)$, in particular replacing some entries $z_{i}$ by $z_{i}^{c}$. We write $\gamma=t_{H} \in T_{H}, \delta=t \in T_{G}$. Note that in $G$, a regular stable conjugacy class is parameterized, modulo conjugacy, by

$$
\operatorname{ker}\left(H^{1}\left(F, T_{G}\right) \longrightarrow H^{1}(F, G)\right) .
$$

Since det : $G \rightarrow U(1)$ induces an isomorphism in cohomology, we see that stable conjugacy coincides with conjugacy. The endoscopic identity then reduces to

$$
O_{t_{H}}\left(f^{H}\right)=\Delta_{0}\left(t_{H}, t\right) O_{t}(f)
$$

for associated elements $\left(t_{H}, t\right)$. Write

$$
\Delta_{0}\left(t_{H}, t\right)=\Delta_{I} \Delta_{I I} \Delta_{I I I, 1} \Delta_{I I I, 2} \Delta_{I V}
$$


Here $\Delta_{I V}=D_{G}(t) / D_{H}\left(t_{H}\right)$, where $D_{G}(t)=\left|\operatorname{det}\left[(\operatorname{Ad}(t)-1) ; \operatorname{Lie}(G) / \operatorname{Lie}\left(T_{G}\right)\right]\right|^{1 / 2}$ is a geometric factor that will occur naturally later and $D_{H}$ is the analogue for $H$. If $T_{s c}=T \cap S U(n)$, the previous remark implies easily that $H^{1}\left(F, T_{s c}\right)=\{1\}$; thus $\Delta_{I}, \Delta_{I I I, 1}$ are trivial (see [CT15, §3.7] for the relevant references to Langlands-Shelstad). The factor $\Delta_{I I}[\mathrm{LS} 87, \mathrm{p} .243]$ requires the choice of data $a_{\alpha} \in E^{\times}$and $\chi_{\alpha}$, a character of $F^{\times}$or $E^{\times}$, depending on whether $\alpha$ is fixed or not by $\operatorname{Gal}(E / F)$, for the (absolute) roots of $\left(G, T_{0}\right)$. We can take all data equal to 1 , giving $\Delta_{I I}=1$.

The amusing factor is $\Delta_{I I I, 2}$, which depends on the embedding of ${ }^{L} H$ in ${ }^{L} G$, and in particular on the choice of the character $\mu$. This is described in [LS87, p. 246]. If $G$ is a quasi-split unitary group and $(T, B)$ is the usual datum of a Borel subgroup and its maximal torus (over $F$ ), the construction of the L-group yields naturally a map $\xi_{T}:{ }^{L} T \rightarrow{ }^{L} G,(t, w) \longmapsto(t, w)\left(t \in \widehat{T}, w \in W_{F}\right)$. (This is associated to trivial $\chi$-data, in the terminology of [LS87].) If $G$ has rank $r$, we take as usual $\widehat{T}=\left(\mathbb{C}^{\times}\right)^{r} \subset \mathrm{GL}_{r}(\mathbb{C})$. This applies to $G$ and $H$. Consider now the embedding $\xi:{ }^{L} H \longrightarrow{ }^{L} G$. We have two tori $T_{H}, T$ and the map $\xi \circ \xi_{T_{H}}: \widehat{T}_{H} \longrightarrow \widehat{T} \subset \widehat{G}$ is $W_{F^{-}}$equivariant. It follows that $\xi \circ \xi_{T_{H}}(t, w)=a(w) \xi_{T}(t, w)$ where $a(w)$ is a 1-cocycle of $W_{F}$ for the action of $W_{F}$ on $\widehat{T}$.

In order to compute $a(w)$, it is convenient to consider the conjugate embedding given by (3.1). Let $\sigma \in W_{F}$ be a representative of $W_{F}-W_{E}$. The cocycle $a(w)$ is the product of a trivial (middle) matrix of size $m$ and an outer matrix matrix $g_{2} \in \mathrm{GL}_{4}(\mathbb{C})$, which we identify with $a(w)$. This outer component is equal to

$$
\left(\begin{array}{llll}
1 & & & \\
& 1 & & \\
& & -1 & \\
& & & -1
\end{array}\right) \text {. }
$$

We find, the other component being trivial:

$$
\begin{aligned}
a(w): W_{F} & \longrightarrow\left(\mathbb{C}^{\times}\right)^{4} \subset \mathrm{GL}_{4}(\mathbb{C}) \\
\sigma & \longmapsto(1,1,-1,-1) \\
w & \longmapsto \mu(w), w \in W_{E} .
\end{aligned}
$$

The corresponding component of $T_{H}=T$ is isomorphic to $\left(E^{\times}\right)^{2}$. Thus $a(w)$ defines componentwise two equal characters of $E^{\times}$, with $\mathrm{L}$-group

$$
\mathbb{C}^{\times} \times \mathbb{C}^{\times}, \sigma(z, w)=\left(w^{-1}, z^{-1}\right)
$$

associated to the cocycle

$$
\begin{aligned}
\alpha: \quad w & \longmapsto(\mu(w), \mu(w)) \quad\left(w \in W_{E}\right) \\
\sigma & \longmapsto((1,-1), \sigma) .
\end{aligned}
$$

By Langlands' description of Shapiro's lemma for L-groups [Lan89], this is associated to the character $\mu(z)$ of $z \in E^{\times}$. We have shown:

Lemma 3.6. Assume $t \in T_{G}$ and $t_{H} \in T_{H} \subset U(m) \times U(4)$ are associated, with $t_{H}=\left(t^{\prime}, z_{1}, z_{2}\right),\left(z_{1}, z_{2}\right) \in$ $E^{\times} \times E^{\times} \subset U(4)$. Then $\Delta\left(t_{H}, t\right)=\mu\left(z_{1}\right) \mu\left(z_{2}\right) \Delta_{I V}\left(t_{H}, t\right)$.

Note that since $\mu\left(\bar{z}^{-1}\right)=\mu(z)$, this does not depend on the choice of the representative $\left(z_{1}, z_{2}\right)$ for the second component.

\subsection{Proof of Theorem 2.3}

Now that the local transfer factors are available to us, we can compare the elements of the local L-packet of Mœglin-Mok (Theorem 3.2) and the representations appearing in Theorem 2.5, thus proving Theorem 2.3 and Theorem 3.3. We recall that $E / F$ is assumed to be ramified, and $F$ of odd residue characteristic.

We first assume $m \geq 5$ and consider the semi-stable modules $X, Y, Z$ of Theorem 2.5 , which we now denote by $X^{\prime}, Y^{\prime}, Z^{\prime}$. With $\varphi_{E}$ as in $\S 3.2$, let $\varphi: W_{F} \longrightarrow{ }^{L} G$ be the associated parameter. The computation of $\mathcal{S}_{\varphi}$ shows that $\varphi$ factors through $\xi:{ }^{L} H \longrightarrow{ }^{L} G$ and therefore defines a parameter $\varphi_{H}$ for $H$, which by 
composition yields the parameter associated to $\mathrm{St}_{m, E} \otimes\left(\operatorname{St}_{1, E} \boxplus \mathrm{St}_{3, E}\right)$ for $H(E)=\mathrm{GL}_{m}(E) \times \mathrm{GL}_{4}(E)$. However, $\xi$ contains, in the component of ${ }^{L} H$ associated to $U(4)$, the character $\mu$, so $\left.\varphi_{H}\right|_{L_{E}}$ is, on this component,

$$
w \longmapsto \mu^{-1}(w)(s p(1) \oplus \operatorname{sp}(3)),
$$

$s p(i)$ being the irreducible representation of degree $i$ of $\mathrm{SU}_{2}(\mathbb{R})$.

Using Mœglin's results [Mœg07] we find that the parameter $s p(1) \oplus s p(3)$ for $U(4)$, originating from unstable base change, is associated to two discrete series representations $\tau_{4}^{+}, \tau_{4}^{-}$of $U(4)$. We want to understand their Jacquet modules (for the Borel subgroup.) The full representation of $G L(4, E), S t(3)_{E} \boxplus$ $S t(1)_{E}$, has a Jacquet module of length 4 , with exponents

$$
[1,0,-1,0],[0,1,0,-1],[1,0,0,-1](\times 2) .
$$

(We have used the natural notation for exponents on $G L(4, E)$, with maximal split torus $\left(E^{\times}\right)^{4}$.) Thus $S t(3)_{E} \boxplus S t(1)_{E}$ is a submodule of the principal series induced from [1,0,0,-1], and it is its only irreducible submodule. This follows for instance from the realisation of the Zelevinsky involution from an involution of the Iwahori-Hecke algebra, since this representation has a unique quotient and the involution sends it to its dual; see [Pro98].

Let us denote, for the end of this proof, by $\theta$ the Galois automorphism of $G L(4, E)$ defined by our unitary group. Then $\theta$ acts naturally on the Jacquet module, the only stable exponent being $[1,0,0,-1]$. As in [Mœg07], the twisted character of $S t(3)_{E} \boxplus S t(1)_{E}$ on the maximally split torus is associated to the character of $\tau_{4}^{+} \oplus \tau_{4}^{-}$on same (for $U(4)$ ). However, the representation induced from $[1,0,0,-1]$ is induced from the $\theta$-stable parabolic of type $(1,2,1)$, from a $\theta$-stable representation. A standard computation then gives its twisted character, which does not vanish on the maximally split torus, and contains the exponent $[1,0]$. Thus $\theta$ has non-zero trace on the subspace of the Jacquet module associated to $[1,0,0,-1]$. This implies that the Jacquet module of $\tau_{4}^{+} \oplus \tau_{4}^{-}$is equal to $2[1,0]$. Twisting by $\mu^{-1}$, we see that the stable packet associated to the component of degree 4 of $\varphi_{H}$ is therefore composed of two representations $\pi_{4}^{+}, \pi_{4}^{-}$ and has Jacquet module

$$
2 \mu^{-1}\left(z_{1} z_{2}\right) \otimes[1,0]
$$

with obvious notation. ${ }^{1}$ Of course, the representation of $U(m) \subset H$ is the Steinberg representation.

Consider now the tempered modules $X^{\prime}, Y^{\prime}$ of Theorem 2.5, with a distinguished exponent $e_{0}=$ $[k-2, k-3, \ldots, 0,-1]$. Each defines, by stable base change, a representation $\Pi$ of $\mathrm{GL}_{n}(E)-$ perhaps not the same $\Pi$. Arguing as in $\left[\mathrm{CT} 15\right.$, Lemma 4.3] we find that $\Pi$ is a semi-stable representation of $\mathrm{GL}_{n}(E)$ (i.e, one having Iwahori-fixed vectors) originating from stable base change. Thus the parameter of $\Pi$ is of the form

$$
\bigoplus_{i} s p\left(n_{i}\right) \otimes \chi_{i}
$$

with $\chi_{i}\left(z^{c}\right)=\chi_{i}\left(z^{-1}\right), \chi_{i}$ unramified, and the usual multiplicity 1 condition - no factor of the form $(s p(n) \otimes$ $\chi) \oplus\left(\operatorname{sp}(n) \otimes \chi\left(z^{-c}\right)\right.$ - since $X^{\prime}, Y^{\prime}$ are in the discrete series as is immediately seen from their exponents. Comparing Jacquet modules (twisted for $\Pi$ ) we see first that the $\chi_{i}$ are trivial - thus $n_{i}$ odd since the parameter is conjugate orthogonal $(\S 2.2)$.

Moreover the $\theta$-stable exponent for $\mathrm{GL}_{n}(E)$ associated to $e_{0}$,

$$
[k-2, \ldots, 0,-1,0,1,0, \ldots, 2-k]
$$

must occur in $\Pi_{N_{0}(E)}^{\text {norm }}$. Since the Jacquet module of $\Pi$ is a sum of $\mathfrak{S}_{n}$-conjugates of the total exponents determined from the segments $\left[\frac{n_{i}-1}{2}, \ldots, \frac{1-n_{i}}{2}\right]$, this implies $\left\{n_{i}\right\}=\{m, 3,1\}$. Consequently $X^{\prime}, Y^{\prime}$ are contained in the L-packet described in Theorem 3.2. We now use the character identities associated to base change. Let $f_{E}$ be a smooth, compactly supported function on $G(E), f$ associated to $f_{E}$ on $G=G(F)$, and $f^{H}$ similarly on $H$. For $\pi \in \Pi_{\varphi}$, let $\varepsilon(\pi)$ be the sign associated to $H$.

The stable identity

$$
\sum_{\pi}\langle\operatorname{trace} \pi, f\rangle=\operatorname{trace}\left(\Pi_{E}\left(f_{E}\right) I_{\theta}\right),
$$

\footnotetext{
${ }^{1}$ We thank, once more, Møglin for this argument.
} 
where $\pi$ runs over $\Pi_{\varphi}$ and $I_{\theta}$ is the Whittaker-normalized intertwining operator associated to base change, tells us little because the twisted trace of $I_{\theta}$ on the Jacquet module of $\Pi_{E}$ involves indeterminate signs on the eigenspaces. (The part of the identity associated to the exponent $e_{0}$ has already been exploited.) Consider now the identity

$$
\sum_{\pi} \varepsilon(\pi)\langle\operatorname{trace} \pi, f\rangle=\left\langle\operatorname{trace} \pi_{H}, f^{H}\right\rangle
$$

where we write, for simplicity, $\pi_{H}$ for the L-packet consisting of $S t_{m} \otimes \pi_{4}^{+}$and $S t_{m} \otimes \pi_{4}^{-} ; S t_{m}$ is the Steinberg representation of the factor $U(m)$; and the Jacquet module of $\pi_{4}^{+} \oplus \pi_{4}^{-}$is equal to $2 \mu^{-1}\left(z_{1} z_{2}\right) \otimes[1,0]$.

We imitate the method of [CT15, §3.7]. For functions $f$ whose orbital integrals are supported on the regular part of $T=T_{G}$ (notation of $\S 3.4$ ) we have

$$
\langle\operatorname{trace} \pi, f\rangle=\int_{T^{+}} \Theta\left(\pi_{N_{0}}^{n o r m}\right)(t) D_{G}(t) O_{t}(f) d t
$$

Similarly

$$
\left\langle\operatorname{trace} \pi_{H}, f^{H}\right\rangle=\int_{T_{H}^{+}} \Theta\left(\pi_{H, N_{H}}^{n o r m}\right) D_{H}\left(t_{H}\right) O_{t_{H}}\left(f^{H}\right) d t_{H} .
$$

Recall that $\Delta^{I V}\left(t_{H}, t\right)=D_{G}(t) / D_{H}\left(t_{H}\right)$. The transfer factor $\Delta\left(t_{H}, t\right)$ contains the character $\mu\left(z_{1} z_{2}\right)$, whose inverse occurs in $\pi_{H}$. Writing $e_{H}=([k-2, k-1, \ldots, 1],[1,0])$ for the exponent of (each component of) $\pi_{H}$ corrected by this transfer factor, we now have, with $g\left(t_{H}\right)=\Delta\left(t_{H}, t\right) O_{t_{H}}\left(f^{H}\right)$ :

$$
\left\langle\operatorname{trace} \pi_{H}, f^{H}\right\rangle=2 \int_{T_{H}^{+}} e_{H}\left(t_{H}\right) g\left(t_{H}\right) d t_{H} .
$$

We recall that $t_{H} \in T_{H}^{+}$is associated to several elements of $T_{G}^{+}$. Neglecting the factor $U(1)$ of both tori (on which our data can be taken equal to 1 ), we can pretend that $T_{G}^{+}$is parameterized by $\left(z_{1}, \ldots z_{k}\right)$ : $\left|z_{1}\right|<\left|z_{2}\right|<\cdots\left|z_{k}\right|<1\left(z_{i} \in E^{\times}\right)$and $T_{H}^{+}$by

$$
\left(z_{1}, \ldots z_{k-2} ; z_{k-1}, z_{k}\right):\left|z_{1}\right|<\cdots<\left|z_{k-2}\right|,\left|z_{k-1}\right|<\left|z_{k}\right|<1 \text {. }
$$

Thus an element of $T_{H}^{+}$is obtained from an element $t \in T_{G}^{+}$by $w \in \mathfrak{S}_{k}, w$ increasing on the two obvious intervals. We see that the alternating sum of exponents given by the left-hand side of (3.7) is equal to $2 \sum_{w} w([k-2, \ldots, 1,1,0])$; here $w$ runs over the specified elements.

Now assume $m=5$, thus $k=4$. The associated sum is easily seen to be equal to

$$
j Y^{\prime}-j X^{\prime}+j Z^{\prime}
$$

( $j=$ normalized Jacquet module), cf. Theorem 2.3. Since the Jacquet modules of $X^{\prime}, Y^{\prime}, Z^{\prime}$ are clearly independent in the Grothendieck group of $T$, we deduce that $Y=Y^{\prime}, X=X^{\prime}, X$ and $Y$ being as in Theorem 3.3 , with $\varepsilon(Y)=1, \varepsilon(X)=-1$, and that the Jacquet module of $\pm(Z-W)$ is $j\left(Z^{\prime}\right)=[1,0,2,1]+[1,2,0,1]$. However, the expression of $j\left(Z^{\prime}\right)$ implies that

$$
Z^{\prime} \hookrightarrow \mathrm{n}-\operatorname{Ind}\left(\mathrm{St}_{2, E}|\cdot|^{1 / 2} \otimes \mathrm{St}_{U(5)}\right),
$$

the induction being unitary, from the Levi subgroup $\mathrm{GL}_{2}(E) \times U(5)$. The induced representation has a unique irreducible submodule. (It is an induced representation, the parameter being in the opposite chamber of that giving a unique Langlands quotient). Since (3.9) is now true for $Z$ or $W$, we see that one of them is $Z^{\prime}$ : it is the representation $Z$ described in Theorem 3.3. Furthermore $\varepsilon(Z)=1$.

For $m>5$, we can separate the set of increasing elements of $\mathfrak{S}_{k}$ in two sets, those fixing 1 and those sending 1 to a larger index (thus $k-2$ to a smaller power). The inductive description of $j\left(X_{k}\right), j\left(Y_{k}\right), j\left(Z_{k}\right)$ in Theorem 3.3 is then immediately seen to be compatible with the signs $\varepsilon(X)=-1, \varepsilon(Y)=1$; and the identification of $Z$ given by the theorem is obtained by the same argument, implying $\varepsilon(Z)=1$. Since $\varepsilon$ is a character of the L-packet, we must have $\varepsilon(W)=-1$ (including for $m=5$ ); the computation of Jacquet modules shows in fact that $W$ is not semi-stable. 
Finally, consider $m=3$. Thus the L-packet of Møglin, Mok has two elements. The exponent $[1,0,1]$ in Theorem 3.3 again shows, by the argument given at the beginning of this paragraph, that both $X^{\prime}$ and $Y^{\prime}$ belong to the $\mathrm{L}$-packet.

Alternately, it would be easy to show that $X^{\prime}$ and $Y^{\prime}$ (described in Theorem 2.5) are the two summands of $\mathrm{n}-\mathrm{Ind}\left(\mathrm{St}_{3, E} \otimes \mathbf{1}\right)$ from the $\mathrm{GL}_{3}(E) \times U(1)$ Levi subgroup of $U(7)$. The endoscopic identity implies $\varepsilon(Y)=1, \varepsilon(X)=-1$ since the transfer to $H$ of $\varepsilon(Y)+\varepsilon(X)$ is a positive sum of representations.

We now complete the identification of $X, Y, \ldots$ by the corresponding characters of $\mathcal{S}_{\phi}$ given after Theorem 3.2. The reader will easily see that this is not necessary for the proof of our main results, only $\varepsilon=\varepsilon_{1} \varepsilon_{2}$ being relevant; we will therefore be brief. We need another endoscopic identity. Let us denote, for this paragraph, by $H$ the endoscopic group of type $(1, n-1)$, thus associated to the character $\varepsilon_{1}$ in $\S 3.2$. There is an endoscopic embedding $\xi:{ }^{L} H \rightarrow{ }^{L} G$ with ${ }^{L} H=\widehat{H} \rtimes W_{F}, \widehat{H}=\mathrm{GL}_{1}(\mathbb{C}) \times \mathrm{GL}_{n-1}(\mathbb{C})$, given by formulas similar to those in 3.1. Here $w \in W_{E}$ is sent to the diagonal matrix $\left(1, \mu(w) 1_{n-1}\right)$. Our parameter $\varphi$ (associated to $\varphi_{E}$ by stable base change) is equal to $\xi \circ \varphi_{H}$, where $\left.\varphi_{H}\right|_{\mathrm{SU}_{2}(\mathbb{R})}$ is conjugate to

$$
s \mapsto\left(1, \operatorname{Sym}^{2} s \oplus \operatorname{Sym}^{m-1} s\right)
$$

and

$$
\left.\varphi_{H}\right|_{W_{E}}: w \mapsto\left(1, \mu(w)^{-1} 1_{n-1}\right) .
$$

In particular the second component of $\varphi_{H}$ defines an unstable discrete L-packet for $U(n-1)$ with two elements $\pi_{1}, \pi_{2}$ [Mœg07]. The extra identity, cf. (3.7), is

$$
\sum_{\pi} \varepsilon_{1}(\pi)\langle\operatorname{trace} \pi, f\rangle=\left\langle\operatorname{trace} \pi_{H}, f^{H}\right\rangle
$$

where $\pi_{H}=\mathbf{1} \otimes\left(\pi_{1} \oplus \pi_{2}\right)$.

As in $§ 3.4$ we consider orbital integrals on $T_{G}, T_{H}$. The transfer factor is computed in the same fashion. Note that $H$, and indeed its factor $U(n-1)$, has the same rank as $G$. Assume $m=5, k=4$. As before we are reduced to a computation on $T_{G}^{+}$and $T_{H}^{+}$, whose descriptions coincide in this case. Moreover the character $\mu$ occurring in the endoscopic functoriality is compensated by the transfer factor, as before. By the comparison, the module $\sum_{\pi} \varepsilon_{1}(\pi) j \pi(\pi=X, Y, Z, W)$ is effective (i.e., a positive sum). This implies by Theorem $2.5 \varepsilon_{1}(Y)=1$, compatibly with the fact that $Y$ is in fact generic. Thus $\varepsilon_{1}(X)$ or $\varepsilon(Z)=-1$. The description of $\varepsilon_{1}$ is complete if we show that $\varepsilon_{1}(X)=1$, implying that $\varepsilon_{1}(Z)=\varepsilon_{1}(W)=-1$. Note that

$$
j(X)+j(Y)-j(Z)
$$

is indeed effective. If $\varepsilon_{1}(X)=-1$, the exponent $[2,1,0,-1]$ disappears in the Jacquet module of $\pi_{H}$. The proof is therefore completed by

Proposition 3.7 (Mœglin). The Jacquet module of $\pi_{H}$ contains, with multiplicity 2, the character

$$
\mu(z)^{-1}[2,1,0,-1]
$$

of $T_{H}$. More generally, assume $n=a+b$ with $a, b$ odd. If $\pi$ is the L-packet constructed from the datum $\left(S t_{a, E} \boxplus S t_{b, E}\right)$, then the full Jacquet module contains

$$
\left[\frac{b-1}{2}, \frac{b-3}{2}, \ldots \frac{1-a}{2}\right](a \leq b) .
$$

Proof. This follows from the arguments of [Mœg07], just as in [CT15, Appendix A]. The (induced) representation associated to the datum $\mathrm{St}_{3, E} \boxplus \mathrm{St}_{3, E}$ ( we neglect the constant twist by $\mu^{-1}$ in the next arguments) has a Jacquet module of exponent $[1,0,-1]$ with multiplicity 2 . It splits into two summands, each having a Jacquet module of dimension 1 with this exponent. For $\mathrm{St}_{5, E} \boxplus \mathrm{St}_{3, E}$, we now use Moglin's hereditary formula for Jacquet modules; with her notation, we have:

$$
\operatorname{Jac}_{||^{2}}^{\widetilde{G}}\left(\mathrm{St}_{5, E} \boxplus \mathrm{St}_{3, E}\right)=\mathrm{St}_{3, E} \boxplus \mathrm{St}_{3, E}
$$


This means here, by the identity between character for $U(8)$ and twisted character, that the exponents of the full L-packet $\pi$ starting with 2 are those of $\mathrm{St}_{3, E} \boxplus \mathrm{St}_{3, E}$ with the same multiplicity, whence the result; the argument in the general case is the same, starting with $\mathrm{St}_{a, E} \boxplus \mathrm{St}_{b, E}$.

Finally, a similar argument applies for $m>5$, using the inductive description of the modules.

\subsection{Compact transfer: proofs}

Returning to the global situation, we are now able to obtain the analogue of Theorem 3.5 for our $\mathbb{R}-$ anisotropic group $G$. We will also need the similar theorem when the datum is a cuspidal representation of $G\left(\mathbb{A}_{E}\right)$. For clarity we assume first that $l>5$ (thus $n=l+2>7$ ).

We fix a CM extension $E / F$ as before, and places $v_{0}, \ldots, v_{s}$ of $F$ which are ramified in $E$ and of odd residue characteristic. We write $w_{0}, \ldots, w_{s}$ for the places of $E$ dividing $v_{0}, \ldots, v_{s}$. We assume $[F: \mathbb{Q}]$ even. Assume given $\sigma_{1}, \sigma_{2}$, two conjugate self-dual, cuspidal representations of $\mathrm{GL}_{4}\left(\mathbb{A}_{E}\right)$ and $\mathrm{GL}_{m}\left(\mathbb{A}_{E}\right)$ respectively. We will assume that at each Archimedean prime $v$, the Langlands parameter of $\sigma_{1} \boxplus \sigma_{2}$ is

$$
z \mapsto \operatorname{diag}\left((z / \bar{z})^{\frac{n-1}{2}},(z / \bar{z})^{\frac{n-3}{2}}, \ldots,(z / \bar{z})^{\frac{1-n}{2}}\right)
$$

cf. $§ 3.3$. (Thus $\sigma_{2}$ is RACSDC, $\sigma_{1}$ is a twist of such by $\mu$.) There is then an element $w_{v} \in \mathfrak{S}_{n}$ conjugating the Langlands parameter $\varphi_{\sigma_{1}} \oplus \varphi_{\sigma_{2}}$ to the Langlands parameter associated to (3.10), as in $\S 3.4$ and [Clo11, p.362]. We assume

$$
\prod_{v \mid \infty} \operatorname{det}\left(w_{v}\right)=1
$$

Note that this does not depend on the prime of $E$ dividing $v$. We assume in addition that all other primes of ramification of $\sigma_{1}, \sigma_{2}$ are above split primes of $F$. If $w$ is a place of $E$ ramified over the place $v$ of $F$, recall that we have associated to the representation

$$
\Pi_{E_{w}}=\mathrm{St}_{m, E_{w}} \boxplus \mathrm{St}_{3, E_{w}} \boxplus \mathrm{St}_{1, E_{w}}
$$

of $\mathrm{GL}_{n}\left(E_{w}\right)$ the $\mathrm{L}-$ packet $\Pi_{\varphi_{v}}$ and a map $\varepsilon_{v}: \Pi_{\varphi_{v}} \longrightarrow\{ \pm 1\}$, with

$$
\varepsilon_{v}\left(X_{v}\right)=\varepsilon_{v}\left(W_{v}\right)=-1, \varepsilon_{v}\left(Y_{v}\right)=\varepsilon_{v}\left(Z_{v}\right)=1 .
$$

Theorem 3.8. $\quad$ 1. Suppose that $\sigma_{1}$ and $\sigma_{2}$ are conjugate self-dual, cuspidal representations of the groups $\mathrm{GL}_{4}\left(\mathbb{A}_{E}\right)$ and $\mathrm{GL}_{m}\left(\mathbb{A}_{E}\right)$, respectively, which satisfy the following conditions:

(a) $\sigma_{1} \boxplus \sigma_{2}$ is regular algebraic of infinity type

$$
((l+1) / 2,(l-1) / 2, \ldots,(-1-l) / 2)
$$

at every infinite place, and (with notation as above) we have $\prod_{v \mid \infty} \operatorname{det}\left(w_{v}\right)=1$.

(b) There exist places $v_{0}, \ldots, v_{s}$ of $F$, ramified in $E$ and of odd residue characteristic, such that for each $i=0, \ldots, s, \sigma_{1, w_{i}} \cong \mathrm{St}_{3, E_{w_{i}}} \boxplus \mathrm{St}_{1, E_{w_{i}}}$, and $\sigma_{2, w_{i}} \cong \mathrm{St}_{m, E_{w_{i}}}$.

(c) Any other place of $E$ at which $\sigma_{1} \boxplus \sigma_{2}$ is ramified is split over $F$. Any place $v \neq v_{0}, \ldots, v_{s}$ of $F$ is unramified in $E$.

Then there exist exactly $4^{s+1} / 2$ automorphic representations $\sigma$ of $G\left(\mathbb{A}_{F}\right)$ with stable base change $\pi=$ $\sigma_{1} \boxplus \sigma_{2}$. They each appear with multiplicity one, and are in bijection with the tuples $\left(\sigma_{v_{i}}\right)_{i=0}^{s} \in \prod_{i=0}^{s} \Pi_{\varphi_{v}}$ satisfying $\prod_{i=0}^{s} \varepsilon_{v_{i}}\left(\sigma_{v_{i}}\right)=1$.

2. Let $\pi$ be a RACSDC automorphic representation of $\mathrm{GL}_{n}\left(\mathbb{A}_{E}\right)$ satisfying the following hypotheses:

(a) $\pi$ is regular algebraic of infinity type

$$
((l+1) / 2,(l-1) / 2, \ldots,(-1-l) / 2)
$$

at every infinite place. 
(b) There exist places $v_{0}, \ldots, v_{s}$ of $F$, ramified in $E$ and of odd residue characteristic, such that for each $i=0, \ldots, s, \pi_{w_{i}} \cong \mathrm{St}_{m, E_{w_{i}}} \boxplus \mathrm{St}_{3, E_{w_{i}}} \boxplus \mathrm{St}_{1, w_{i}}$.

(c) Any other place of $E$ at which $\pi$ is ramified is split over $F$. Any place $v \neq v_{0}, \ldots, v_{s}$ of $F$ is unramified in $E$.

Then there are exactly $4^{s+1}$ automorphic representations $\sigma$ of $G\left(\mathbb{A}_{F}\right)$ with stable base change $\pi$, each appearing with multiplicity one. They are naturally in bijection with tuples $\left(\sigma_{v_{i}}\right)_{i=0}^{s} \in \prod_{i=0}^{s} \Pi_{\varphi_{v_{i}}}$.

We note that over any finite place of $\mathrm{F}$ different from $v_{0}, \ldots, v_{s} \mathrm{E} / \mathrm{F}$ is unramified or split, and there is only one element in the local L-packet for $G\left(F_{v}\right)$ associated to $\sigma_{1} \boxplus \sigma_{2}$ or $\pi$. (In the inert case, note that there is only, up to conjugacy, one hyperspecial group for $G\left(F_{v}\right)$.)

Remark 3.9. The condition $\prod_{v \mid \infty} \operatorname{det}\left(w_{v}\right)=1$ in the first part of the theorem is implied by the following two assumptions:

1. For every place $v$ of $F$ dividing $l, v$ splits in $E$ and $\left[F_{v}: \mathbb{Q}_{l}\right]$ is even.

2. There exists an isomorphism $\iota: \overline{\mathbb{Q}}_{l} \cong \mathbb{C}$ such that $\sigma_{1} \boxplus \sigma_{2}$ is $\iota$-ordinary.

Indeed, if $\tau: E \hookrightarrow \mathbb{C}$ is an embedding inducing the place $v$ of $F$, then $\operatorname{det}\left(w_{v}\right)$ depends (by [CT14, Lemma 2.6]) only on the place of $F$ dividing $l$ induced by the embedding $\iota^{-1} \tau: E \hookrightarrow \overline{\mathbb{Q}}_{l}$. Since we are assuming $\left[F_{v}: \mathbb{Q}_{l}\right]$ to be even, the signs in the product $\prod_{v \mid \infty} \operatorname{det}\left(w_{v}\right)$ must cancel, so the condition $\prod_{v \mid \infty} \operatorname{det}\left(w_{v}\right)=1$ holds. We will assume these extra conditions in the applications in $\S 4$ below. The same remarks apply to Theorem 3.10 below.

This can also be seen in terms of Shelstad's formalism for endoscopy, cf. [She82, She10, She], since $\prod_{v \mid \infty} \operatorname{det} w_{v}$ equals $\prod_{v \mid \infty} \chi\left(g_{v} s g_{v}^{-1}\right)$, where:

- $g_{v} \in \widehat{G}$ conjugates $\xi \circ \varphi_{H, v}$ to a Langlands parameter whose restriction to $\mathbb{C}^{\times}$is diagonal and dominant for the upper-triangular Borel, so that the centralizer of $\operatorname{Ad}\left(g_{v}\right) \circ \xi \circ \varphi_{H, v}$ is the subgroup $\operatorname{diag}( \pm 1, \ldots, \pm 1)$ of $\widehat{G}$ having $2^{n}$ elements.

- $\chi$ is the character $\left((-1)^{(n-1) / 2},(-1)^{(n+1) / 2},(-1)^{(n-1) / 2}, \ldots\right)$ (alternating signs) of this group ( $\chi$ is trivial on $\left.Z_{\widehat{G}}^{\operatorname{Gal}\left(\bar{F}_{v} / F_{v}\right)}=\{ \pm 1\}\right)$.

Compare [Mok15, §7.1], in particular Theorem 7.1.1, and the reference to Shelstad's work there.

The proof of Theorem 3.8 will rely on Theorem 3.5. Consider first the quasi-split group $G^{*}$. By Arthur's stabilization of the trace formula [Art03] (we use Arthur's formulation in [Art05]) we have, for a function $f_{*}=\bigotimes_{v} f_{*, v}$ on $G^{*}\left(\mathbb{A}_{F}\right)$ :

$$
I_{\text {disc }}^{G^{*}}\left(f_{*}\right)=\sum_{\mathcal{E}} \iota\left(G^{*}, \mathcal{E}\right) S T_{\text {disc }}^{\mathcal{E}}\left(f_{*}^{\mathcal{E}}\right)
$$

where $\mathcal{E}$ runs over the equivalence classes of elliptic endoscopic data, including $H$. We refer to Arthur for the precise definitions; here $I_{\text {disc }}^{G^{*}}$ denotes the discrete part of the trace formula for $G^{*}$, while $S T_{\text {disc }}^{\mathcal{E}}$ is a stabilised form of this expression, for the endoscopic group associated to $\mathcal{E}$.

Similarly, for $f$ a function on $G\left(\mathbb{A}_{F}\right)$ :

$$
T_{\text {disc }}^{G}(f)=I_{\text {disc }}^{G}(f)=\sum_{\mathcal{E}} \iota(G, \mathcal{E}) S T_{\text {disc }}^{\mathcal{E}}\left(f^{\mathcal{E}}\right) .
$$

An argument already introduced in [CHL11] implies that the only relevant data, when we consider representations with base change $\pi$, are $\left(G^{*}, H\right)$. The argument is the same as in [CHL11, Lemma 4.2]. By the main result in [Lab11], each term in $S T_{\text {disc }}^{\mathcal{E}}\left(f^{\mathcal{E}}\right)$ in (3.13) is associated by base change - say, at almost all primes to 'discrete', cohomological automorphic representations of the group $H^{\prime}\left(\mathbb{A}_{E}\right)=G L_{a}\left(\mathbb{A}_{E}\right) \times G L_{b}\left(\mathbb{A}_{E}\right)$ stable by the Galois automorphism associated to the unitary group $H^{\prime}$ deduced from $\mathcal{E}$. The meaning of 'discrete' 
here is precisely explained in [CHL11, p. 486]: they are discrete parameters for descent to the unitary group. For each block $G L_{a}$ or $G L_{b}$, they are Langlands sums of cuspidal representations, or more generally of Speh representations. Since our representation $\pi$ is a Langlands sum of two cuspidal representations of $G L_{4}$ and $G L_{m}$, we see first that the 'discrete' representations must be sums of cuspidal representations, and then, by the disjunction results of Jacquet-Shalika [JS81b, JS81a] that the only possiblities for $H^{\prime}$ are as indicated.

The functions $f$ and $f_{*}$ coincide at finite primes. In (3.13), $f^{G^{*}}$ is associated to $f$, and we take it of course to be equal to $f_{*}$. At the Archimedean primes, $f_{v}$ can be taken to be the constant function equal to 1. Assume $\pi_{\infty}=\otimes \pi_{v}$ is a discrete series representation of $G^{*}\left(F_{\infty}\right)$, in the L-packet associated to the trivial representation. Let $f_{*, \infty}$ be a pseudo-coefficient of $\pi_{\infty}$ : thus trace $\pi_{\infty}\left(f_{*, \infty}\right)=1$. It is well-known that $f_{*, \infty}$ is associated to the constant function 1 on $G\left(F_{\infty}\right)$. By our condition on the Weyl element $w$, we have, $\Pi(H)$ being the L-packet of discrete series for $H$ associated to $\sigma_{1} \mu^{-1} \boxplus \sigma_{2}$ :

$$
\left\langle\text { trace } \Pi(H), f_{\infty}^{H}\right\rangle=\left\langle\text { trace } \mathbb{C}, f_{\infty}\right\rangle=1 \text {. }
$$

By the endoscopic identity for $\left(H, G^{*}\right)$ at the Archimedean prime,

$$
\left\langle\operatorname{trace} \Pi(H), f_{*, \infty}^{H}\right\rangle=\sum_{\pi}\langle s, \pi\rangle\left\langle\operatorname{trace} \pi, f_{*, \infty}\right\rangle,
$$

where $\pi$ runs over the L-packet of discrete series representations of $G^{*}\left(F_{\infty}\right)$ associated to the trivial representation of $G\left(F_{\infty}\right)$. By assumption this is equal to $\left\langle s, \pi_{\infty}\right\rangle$ for our chosen representation $\pi_{\infty}$.

Consider now another $L$-packet $\Pi_{1}(H)$ transferring to this same $L$-packet (cf. [Clo11]). Then the corresponding automorphic representations of $H$ give rise to Galois representations having different HodgeTate weights [Gue11, Theorem 2.3] ${ }^{2}$. We now introduce the family $\psi$ of Hecke eigenvalues, at (almost all) unramified primes, corresponding to $\pi$. By unramified functoriality, it defines such a family for all the endoscopic groups. They are associated to the corresponding Galois representations. By the Chebotarev density theorem applied to these representations, we see that their contributions to the traces vanish once we consider the parts of the trace formulas associated to $\psi$. We denote these terms by a lower index $\psi$.

Assuming $\pi_{\infty}$ chosen so that $\left\langle s, \pi_{\infty}\right\rangle=1$ we see that $f_{\infty}^{H}$ can be chosen equal to $f_{*, \infty}^{H}$. We now have

$$
I_{\text {disc }}^{G^{*}}\left(f_{*}\right)_{\psi}=T_{\text {disc }}^{G}(f)_{\psi} .
$$

Since $\left\langle s, \pi_{\infty}\right\rangle=1$, Mok's theorem (here Theorem 3.5) implies that there are $4^{s+1} / 2$ representations of $G^{*}\left(\mathbb{A}_{F}\right)$ in the discrete or cuspidal spectrum with Archimedean component $\pi_{\infty}$ and with stable base change $\pi$. Recall that $I_{d i s c}^{G^{*}}$ contains, in addition to the trace $T_{\text {disc }}^{G^{*}}$ in the discrete spectrum, terms associated to Eisenstein series whose parameters are fixed by certain Weyl groups elements, see [Art05, (21.19)]. However the family of Hecke eigenvalues for $G^{*}$, at the unramified primes, determined by $\sigma_{1} \boxplus \sigma_{2}$, is easily seen to be distinct from that of any Eisenstein representation. Thus we actually get the identity

$$
T_{\text {disc }}^{G^{*}}\left(f_{*}\right)_{\psi}=T_{d i s c}^{G}(f)_{\psi}
$$

Since the groups coincide at the finite primes, and $f_{v}$ is the constant function equal to 1 at the Archimedean primes, the identity of traces implies the result.

The proof of the second part of Theorem 3.8 is similar. In this case, after separation of Hecke eigenvalues, only the terms of (3.12) and (3.13) given by $G^{*}$ have to be considered. We take for $f_{*, \infty}$ any pseudo-coefficient of the L-packet. The global datum being stable, the pairing $\langle s, \pi\rangle$ is, at all primes, the trivial character. The result follows.

We note that the same arguments imply that the (strong) stable base change of an automorphic representation $\sigma$ of $G\left(\mathbb{A}_{F}\right)$ exists: it is a representation $\pi=\boxplus_{i} \pi_{i}$ of $\mathrm{GL}_{n}\left(\mathbb{A}_{E}\right)$ with local components at all primes predicted by Langlands functoriality, well-defined if the local components of $\sigma$ are tempered. Indeed recall that there is an $n$-dimensional semi-simple $l$-adic representation associated to $\sigma$ [Gue11, $\S 2]$. We will only be interested in the case where this representation is irreducible or with irreducible factors $(4, m)$. In this case it is easy to see that $\sigma$ is associated to such a representation $\pi$ with either $\pi$ cuspidal or two cuspidal

\footnotetext{
${ }^{2}$ Guerberoff assumes that $E / F$ is unramified at finite primes. It is easy to see that this assumption is irrelevant here.
} 
summands $\pi_{i}$ of sizes $(4, m)$. Consider again the formulas (3.12), (3.13). If $\pi$ is associated to an irreducible Galois representation, standard arguments [CHL11] show that both sums reduce to the stable trace when we consider the part relative to the given Hecke eigenvalues almost everywhere. Now the previous arguments imply that these sums are equal, and this implies that strong, stable base change for $G$ follows from the analogous result for $G^{*}$ proved by Mok: see [Mok15, Theorem 2.5.2].

Assume the semi-simplification is of type $(4, m)$. Then only the terms relative to $G^{*}$ and $H$ have to be compared, and we are, as above, reduced to the term relative to $H$. We must compare the Archimedean functions $f_{\infty}^{H}, f_{*, \infty}^{H}$. The function $f_{\infty}^{H}$ is defined by

$$
\left\langle\operatorname{trace} \Pi(H), f_{\infty}^{H}\right\rangle=\operatorname{det}(w)
$$

for any L-packet $\Pi(H)$ for $\mathrm{H}$ such that $\xi \circ \varphi_{H}=\varphi_{G}, \varphi_{G}$ being associated to the trivial representation. Similarly $f_{*, \infty}^{H}$ is defined by

$$
\left\langle\operatorname{trace} \pi(H), f_{*, \infty}^{H}\right\rangle=\operatorname{det}(w)\left\langle a_{w \tau}, s\right\rangle,
$$

cf. [Clo11, p. 366]. Here $\tau \in \Omega / \Omega_{\mathbb{R}}=\mathfrak{S}_{n} /\left(\mathfrak{S}_{r} \times \mathfrak{S}_{r+1}\right)(n=2 r+1)$ parameterizes a discrete series representation for $G^{*}$ in the L-packet of the trivial representation, and $f_{*, \infty}$ is, as before, a pseudo-coefficient of this discrete series. Fix such a $\sigma$ occurring in $T_{d i s c}^{G}(f)$, and therefore a sequence of Hecke eigenvalues at the unramified primes, and assume the corresponding $H$-term is non-zero. By base change (cf. [Lab11, Theorem 5.1], which has already been used through Guerberoff's result quoted above) there is a corresponding representation $\pi$ of $H\left(\mathbb{A}_{E}\right)$, uniquely defined. This determines the infinity type of $\pi$, and therefore the Lpacket $\Pi(H)$, hence $w$.

Recall that $w \in \Omega \mapsto a_{w}$ is a 1-cocycle of $\Omega$ with values in $H^{1}(\mathbb{R}, T)$ where $T$ is the diagonal anisotropic torus in $G^{*}$ (we refer to [Clo11] for the relevant choices, implicit throughout this paper as in [CT15]). In particular $a_{w}=1$ if $w=1$. If we choose $\tau$ such that $\left\langle a_{w \tau}, s\right\rangle=1$, we deduce that after separation of the family $\psi$ of Hecke eigenvalues corresponding to $\sigma$,

$$
S T_{\text {disc }}^{H}\left(f_{*}^{H}\right)_{\psi}=S T_{\text {disc }}\left(f^{H}\right)_{\psi} .
$$

Finally we have, for this choice of $f_{\infty}, f_{*, \infty}$ :

$$
T_{\text {disc }}(f)_{\psi}=T_{\text {disc }}\left(f_{*}\right)_{\psi}
$$

Note that the $f_{v}$ for finite primes, outside the set specifying $\psi$, are arbitrary. Mok's results, specifically [Mok15, Theorem 2.5.2] now applied to base change to $G(E)$ rather than descent, imply the existence of a stable base change $\pi$ of $\sigma$ - a RACSD representation of $G\left(\mathbb{A}_{E}\right)$ - with local components in the correct Lpackets at all finite primes; at infinite primes $\pi$ is cohomological and its infinitesimal character is associated to the trivial representation of $G\left(F_{\infty}\right)$. Thus $\pi$ is, at all primes, the stable base change of $\sigma$.

Consider now the case where $l=5$, hence $n=7$. The assumptions and data are the same, except that the L-packet associated to $\mathrm{St}_{3, E_{w}} \boxplus \mathrm{St}_{3, E_{w}} \boxplus \mathrm{St}_{1, E_{w}}$ has now two elements $X_{v}, Y_{v}$ (Theorem 3.3). We define a local sign by the formula $\varepsilon_{v}\left(X_{v}\right)=-1, \varepsilon_{v}\left(Y_{v}\right)=1$. Again, the data determine elements $w_{v} \in \mathfrak{S}_{7}$, and we assume $\prod_{v \mid \infty} \operatorname{det}\left(w_{v}\right)=1$. Exactly the same argument now yields:

Theorem 3.10. 1. Suppose that $\sigma_{1}, \sigma_{2}$ are conjugate self-dual, cuspidal representations of $\mathrm{GL}_{4}\left(\mathbb{A}_{E}\right)$, $\mathrm{GL}_{3}\left(\mathbb{A}_{E}\right)$, respectively satisfying the following hypotheses:

(a) $\sigma_{1} \boxplus \sigma_{2}$ is regular algebraic of infinity type

$$
(3,2, \ldots,-3)
$$

at every infinite place, and (with notation as above) we have $\prod_{v \mid \infty} \operatorname{det}\left(w_{v}\right)=1$.

(b) There exist places $v_{0}, \ldots, v_{s}$ of $F$, ramified in $E$ and of odd residue characteristic, such that for each $i=0, \ldots, s, \sigma_{1, w_{i}} \cong \mathrm{St}_{3, E_{w_{i}}} \boxplus \mathrm{St}_{1, E_{w_{i}}}$, and $\sigma_{2, w_{i}} \cong \mathrm{St}_{3, E_{w_{i}}}$. (Here we write $w_{i}$ for the place of $E$ above $v_{i}$.) 
(c) Any other place of $E$ at which $\sigma_{1} \boxplus \sigma_{2}$ is ramified is split over $F$. Any place $v \neq v_{0}, \ldots, v_{s}$ of $F$ is unramified in $E$.

Then there exist exactly $2^{s}$ automorphic representations $\sigma$ of $G\left(\mathbb{A}_{F}\right)$ with stable base change $\pi=\sigma_{1} \boxplus \sigma_{2}$. They each appear with multiplicity one, and are in bijection with the tuples $\left(\sigma_{v_{i}}\right)_{i=0}^{s} \in \prod_{i=0}^{s} \Pi_{\varphi_{v_{i}}}$ satisfying $\prod_{i=0}^{s} \varepsilon_{v_{i}}\left(\sigma_{v_{i}}\right)=1$.

2. Let $\pi$ be a RACSDC automorphic representation of $\mathrm{GL}_{n}\left(\mathbb{A}_{E}\right)$ satisfying the following hypotheses:

(a) $\pi$ is regular algebraic, of infinity type

$$
(3,2, \ldots,-3)
$$

at every infinite place.

(b) There exist places $v_{0}, \ldots, v_{s}$ of $F$, ramified in $E$ and of odd residue characteristic, such that for each $i=0, \ldots, s, \pi_{w_{i}} \cong \mathrm{St}_{3, E_{w_{i}}} \boxplus \mathrm{St}_{3, E_{w_{i}}} \boxplus \mathrm{St}_{1, E_{w_{i}}}$.

(c) Any other place of $E$ at which $\pi$ is ramified is split over $F$. Any place $v \neq v_{0}, \ldots, v_{s}$ of $F$ is unramified in $E$.

Then there are exactly $2^{s+1}$ automorphic representations $\sigma$ of $G\left(\mathbb{A}_{F}\right)$ with stable base change $\pi$, each appearing with multiplicity one. They are naturally in bijection with tuples $\left(\sigma_{v_{i}}\right)_{i=0}^{s} \in \prod_{i=0}^{s} \Pi_{\varphi_{v_{i}}}$.

Finally, the same arguments as in the previous case show that stable base change obtains for representations of $G\left(\mathbb{A}_{F}\right)$.

\section{Level-raising and algebraic modular forms}

This section is devoted to the key level-raising argument. In this introduction we still sketch it in the case of $\mathrm{Sym}^{8}$ and $l=7$.

Assume, at one ramified place $v$ (for $E / F$ ), that our given representation of $\mathrm{GL}_{2}\left(\mathbb{A}_{F}\right)$ (for which we hope to establish the existence of the symmetric power lifting) is Steinberg. Then the local component at $v$ of the representation of $G\left(\mathbb{A}_{F}\right)$ constructed in $\S 3$ will base change to $\mathrm{St}_{3, E_{w}} \boxplus \mathrm{St}_{1, E_{w}} \boxplus \mathrm{St}_{5, E_{w}}$, and therefore lie in the L-packet of representations which is described by Theorem 2.3 .

The flexibility needed in $\S 6$ forces us, in fact, to consider a large number $v_{0}, \ldots, v_{s}$ of such ramified primes. We also assume $q_{v_{0}} \equiv-1 \bmod l$. We can define two level subgroups $U_{0}, U_{1}$ of $G\left(\mathbb{A}_{F}^{\infty}\right)$; at the places $v_{i} \neq v_{0}$ their local factor is the group $\mathfrak{B}$ considered in $\S 2$, which contains an Iwahori subgroup with index 2. At $v_{0}, U_{1}$ is again this group and $U_{0}$ is the parahoric subgroup $\mathfrak{P}$ such that $\operatorname{dim} X^{\mathfrak{P}}=1$ for the distinguished module $X$ of $\S 2$.

The global L-packet constructed in $\S 3$ determines a character of an unramified Hecke algebra that, considered mod $l$, defines a maximal ideal $\mathfrak{m}$. Let $K / \mathbb{Q}_{l}$ be a sufficiently large extension. For $A=K, \mathcal{O}, k$ (integers and residue field of $K$ ), we have the (finite) spaces of automorphic forms on $G$, localized, $S\left(U_{i}, A\right)_{\mathfrak{m}}$ $(i=0,1)$. There is a natural injection $S\left(U_{0}\right) \rightarrow S\left(U_{1}\right)$.

The first main result of this section, Proposition 4.1, controls the Jordan decomposition of a local component $\Sigma_{v}\left(v=v_{0}, \ldots, v_{s}\right)$ occurring in characteristic 0 , so essentially in $S\left(U_{i}, \mathcal{O}\right)_{\mathfrak{m}}$. After base change to $E$, it can obviously be of type $(1,3,5)$; the only other possibilities are $(7,1,1)$ and $(9)$ (the last corresponding to a twist of Steinberg). This follows from the restrictions on the degeneracy mod $l$ of Jordan matrices, and the existence of the Galois representations associated to primes of the Hecke algebra above $\mathfrak{m}$.

The second main result - the level-raising result — consists in showing that (at least one of) these second Jordan decompositions do occur. In other terms, we must rule out the case where all (base changed) representations associated to $\mathfrak{m}$ are of type $(1,3,5)$. These are exactly the local representations of the groups $G\left(F_{v}\right)$ studied in $\S \S 2-3$. The argument is based on the following facts:

1. There is a perfect pairing $S\left(U_{1}, A\right)_{\mathfrak{m}} \times S\left(U_{1}, A\right)_{\mathfrak{m}} \vee \rightarrow A$ for $A=\mathcal{O}$ or $A=k$. (Here $\mathfrak{m}^{\vee}$ is the maximal ideal associated to the contragredient representation.) 
2. For $A=k$, this pairing vanishes on $S\left(U_{0}, k\right)_{\mathfrak{m}} \subset S\left(U_{1}, k\right)_{\mathfrak{m}}$. This is a direct consequence of our assumption $q_{v_{0}} \equiv-1 \bmod l$.

Recall that the module $X$ has a distinguished exponent $e=[2,1,0,-1]$, which occurs in $X_{N_{0}}^{\text {norm }} \cong X^{\mathfrak{B}}$, this space being one-dimensional. We can refine the localization of $S\left(U_{i}, k\right)$ at $\mathfrak{m}$ by using the action of Bernstein's maximal abelian subalgebra of the Iwahori-Hecke algebra, the characters of which are exactly the exponents. Now, using this decomposition for the semi-stable modules $X, Y, Z$ of $\S 2$, we are able to conclude that the isotropic subspace $S\left(U_{0}, k\right)$ (localized at $\mathfrak{m}$ and $e$, or rather $\bar{e}=e \bmod l$ ), is too large, by using Mok's multiplicity formula. We refer the reader to the end of $\S 4$ for the precise argument.

It is worth comparing the work we do here with a theorem of the second-named author [Tho14], which (loosely speaking) allows one to replace a local Weil-Deligne representation $(r, N)$, where $N$ has two Jordan blocks, with a Weil-Deligne representation where $N$ has a single Jordan block (and is therefore regular nilpotent). Unfortunately the methods of [Tho14] require that the residue characteristic $l$ satisfy some stringent conditions (in particular, it should be banal for the local group at the prime where levelraising occurs). Since the strategy of this paper requires us to work with relatively small primes $l$, these methods can not be applied here.

We also note that the argument sketched above is not alone sufficient to obtain a representation which is Steinberg locally, in contrast to the analogous argument in [CT15]; although we show that one of the possibilities $(7,1,1)$ and $(9)$ does occur, we are not able to decide which, and we need the latter to occur in order to able to apply the automorphy lifting theorem proved in [Tho15]. We must therefore take up again the problem of level-raising in the next $\S 5$, where we use Galois-theoretic techniques to obtain the necessary improvements to the results of this section.

\subsection{Set-up}

Let $l \geq 5$ be a prime, and choose an isomorphism $\iota: \overline{\mathbb{Q}}_{l} \cong \mathbb{C}$. Let $E$ be an imaginary CM field with maximal totally real subfield $F$, and satisfying the following conditions:

1. Each place $v \mid l$ of $F$ is split in $E$, and the local degree $\left[F_{v}: \mathbb{Q}_{l}\right]$ is even.

2. There exist places $v_{0}, \ldots, v_{s}$ of $F$, ramified in $E$ and of odd residue characteristic, such that $q_{v_{0}} \equiv$ $-1 \bmod l$ and each $q_{v_{i}}, i=1, \ldots, s$, is a primitive root modulo $l$. Every other place of $F$ is unramified in $E$. We write $w_{0}, \ldots, w_{s}$ for the places of $E$ dividing $v_{0}, \ldots, v_{s}$. (We assume $s \geq 1$.)

Let $n=l+2, m=l-2$. In this case there exists a unitary group $G$ in $n$ variables over $F$, determined uniquely up to isomorphism by the following conditions:

1. The group $G$ is split by $E$.

2. $G\left(F \otimes_{\mathbb{Q}} \mathbb{R}\right)$ is compact.

3. $G$ is quasi-split at each finite place.

We suppose given cuspidal, conjugate self-dual automorphic representations $\pi_{1}$ and $\pi_{2}$ of $\mathrm{GL}_{m}\left(\mathbb{A}_{E}\right)$ and $\mathrm{GL}_{4}\left(\mathbb{A}_{E}\right)$, respectively, that satisfy the following conditions:

1. For each $i=0, \ldots, s$, we have $\pi_{1, w_{i}} \cong \mathrm{St}_{m, E_{w_{i}}}$ and $\pi_{2, w_{i}} \cong \mathrm{St}_{3, E_{w_{i}}} \boxplus \mathrm{St}_{1, E_{w_{i}}}$. If $w \neq w_{0}, \ldots, w_{s}$ is any place of $E$ such that $\pi_{1, w}$ or $\pi_{2, w}$ is ramified, then $w$ is split over $F$.

2. $\pi=\pi_{1} \boxplus \pi_{2}$ is regular algebraic and $\iota$-ordinary, of infinity type $\boldsymbol{a}=\left(a_{\tau}\right)_{\tau \in \operatorname{Hom}(E, \mathbb{C})}$ :

$$
a_{\tau}=((n-1) / 2,(n-3) / 2, \ldots,(1-n) / 2) .
$$

The residual representation $\overline{r_{\iota}(\pi)}$ is a direct sum of two absolutely irreducible constituents $\bar{\rho}_{1}$ and $\bar{\rho}_{2}$. We have $\operatorname{dim} \bar{\rho}_{1}=m, \operatorname{dim} \bar{\rho}_{2}=4$. (We remind the reader that $r_{\iota}(\pi)$ is the Galois representation associated to the regular algebraic automorphic representation $\pi$; see $\S 1.1$ for our conventions, which are those of [CT14].) 
3. For each $i=0, \ldots, s$, let $t_{w_{i}} \in G_{E_{w_{i}}}$ denote a generator of tame inertia. Then the element $\bar{\rho}_{1}\left(t_{w_{i}}\right) \in$ $\mathrm{GL}_{m}\left(\overline{\mathbb{F}}_{l}\right)$ is a regular unipotent element (having a single Jordan block). The element $\bar{\rho}_{2}\left(t_{w_{i}}\right) \in \mathrm{GL}_{4}\left(\overline{\mathbb{F}}_{l}\right)$ has Jordan decomposition corresponding to the partition $4=3+1$.

In this case there exists, by the discussion in $\S 3.6$, an automorphic representation $\sigma$ of $G\left(\mathbb{A}_{F}\right)$ with stable base change $\pi$. We may suppose that, in the notation of $\S 3.6$, we have $\sigma_{v_{0}} \cong X_{v_{0}}$ and $\sigma_{v_{i}}$ is semi-stable for each $i=1, \ldots, s$; for example, we can choose $\sigma_{v_{1}}=X_{v_{1}}$ and $\sigma_{v_{i}}=Y_{v_{i}}$ for each $i \geq 2$. In conjunction with Theorem 2.3, this ensures that we can choose a subgroup $U_{0}$ satisfying the condition 5 below.

Let $K \subset \overline{\mathbb{Q}}_{l}$ be a finite extension of $\mathbb{Q}_{l}$ with ring of integers $\mathcal{O}$, maximal ideal $\lambda$, and residue field $k$. If $U=\prod_{v} U_{v} \subset G\left(\mathbb{A}_{F}^{\infty}\right)$ is an open compact subgroup, then we can define for any $\mathcal{O}$-algebra $A$ the space $S(U, A)$ of algebraic modular forms with level $U$, weight zero, and coefficients in $A$, as the set of functions $G(F) \backslash G\left(\mathbb{A}_{F}^{\infty}\right) / U \rightarrow A$. We choose as in $[\mathrm{CT} 15, \S 3.3]$ the following auxiliary data:

1. An integral model for $G$ over $\mathcal{O}_{F}$.

2. If $w$ is a place of $E$ split over the place $v$ of $F$, an isomorphism $\iota_{w}: G\left(F_{v}\right) \cong \mathrm{GL}_{n}\left(E_{w}\right)$.

3. For each place $v \mid l$ of $F$, a place $\widetilde{v}$ of $E$ above it, and a uniformizer $\varpi_{\widetilde{v}}$ of $\mathcal{O}_{E_{\tilde{v}}}$.

We fix an identification between each group $G_{F_{v_{i}}}$ and the quasi-split group $U_{n}$ of $\S 2$. Having made this choice, the open compact subgroups $\mathfrak{B}_{v_{i}} \subset \mathfrak{P}_{v_{i}} \subset G\left(F_{v_{i}}\right)$ are defined. We now choose an open compact subgroup $U_{0}=\prod_{v} U_{0, v} \subset G\left(\mathbb{A}_{F}^{\infty}\right)$ satisfying the following properties:

1. $U_{0, v_{0}}=\mathfrak{P}_{v_{0}}$.

2. If $i=1, \ldots, s$ then $U_{0, v_{i}}=\mathfrak{B}_{v_{i}}$.

3. If $v$ is a place of $F$ inert in $E$, then $U_{v}$ is a hyperspecial maximal compact subgroup of $G\left(F_{v}\right)$.

4. There exists $c>0$ such that $U_{0, v}=\iota_{\widetilde{v}}^{-1} \operatorname{Iw}_{c}(\widetilde{v})$ for each place $v \mid l$, the subgroup $\operatorname{Iw}_{\widetilde{v}}(c)$ being as in $[\mathrm{CT} 15, \S 3.3]$.

5. $\sigma^{U_{0}} \neq 0$ and $U_{0}$ is sufficiently small (i.e. some $U_{0, v}$ contains no element of finite order).

We define $U_{1}=\prod_{v} U_{1, v}$ by setting $U_{1, v_{0}}=\mathfrak{B}_{v_{0}}$ and $U_{1, v}=U_{0, v}$ if $v \neq v_{0}$. Then $U_{0}$ contains $U_{1}$ with index $q_{v_{0}}+1$.

The Hecke operators $U_{v}^{j}$ at places $v \mid l$, as defined in $[\mathrm{CT} 15, \S 3.3]$, act on $S\left(U_{0}, \mathcal{O}\right)$ and $S\left(U_{1}, \mathcal{O}\right)$. If $U=U_{0}$ or $U_{1}$ we define the Hecke algebra $\mathbb{T}(U, \mathcal{O}) \subset \operatorname{End}_{\mathcal{O}}(S(U, \mathcal{O}))$ as the commutative $\mathcal{O}$-subalgebra generated by the unramified Hecke operators at places $v$ of $F$ split in $E$ such that $U_{v}$ is a maximal compact subgroup, together with the operators $U_{v}^{j}$ at places $v \mid l$. The operators $U_{v}^{j}$ depend on the choice of uniformizer $\varpi_{\widetilde{v}}$, but the algebra $\mathbb{T}(U, \mathcal{O})$ does not.

If $\Sigma$ is an automorphic representation of $G$ such that $\Sigma_{\infty}$ is the trivial representation, then there is a canonical embedding $\iota^{-1} \Sigma^{\infty, U} \hookrightarrow S(U, \mathcal{O}) \otimes_{\mathcal{O}} \overline{\mathbb{Q}}_{l}=S\left(U, \overline{\mathbb{Q}}_{l}\right)$. The subspace $\iota^{-1} \Sigma^{\infty, U \text {,ord }} \subset \iota^{-1} \Sigma^{\infty, U}$ is defined, as in [CT15, §3.3]. If $N \subset S(U, \mathcal{O})$ is an $\mathcal{O}$-submodule such that

$$
N \cap \iota^{-1} \Sigma^{\infty, U, \text { ord }} \neq 0
$$

inside $S\left(U, \overline{\mathbb{Q}}_{l}\right)$, then we say that $\Sigma$ contributes to $N$. In particular, if $\Sigma$ contributes to $S(U, \mathcal{O})$, then $\Sigma^{\infty, U} \neq 0$ and $\Sigma$ is $\iota$-ordinary.

After possibly enlarging $K$, we can assume that representation $\sigma$ contributes to $S\left(U_{0}, \mathcal{O}\right)$, in the above sense, and therefore defines a homomorphism $\mathbb{T}\left(U_{0}, \mathcal{O}\right) \rightarrow \mathcal{O}$, and we write $\mathfrak{m}$ for the kernel of the composite homomorphism $\mathbb{T}\left(U_{1}, \mathcal{O}\right) \rightarrow \mathbb{T}\left(U_{0}, \mathcal{O}\right) \rightarrow \mathcal{O} \rightarrow k$. The dual representation $\sigma^{\vee}$ also contributes to $S\left(U_{0}, \mathcal{O}\right)$, and we write $\mathfrak{m}^{\vee} \subset \mathbb{T}\left(U_{1}, \mathcal{O}\right)$ for the corresponding maximal ideal.

Proposition 4.1. Suppose that $\Sigma$ is an automorphic representation of $G\left(\mathbb{A}_{F}\right)$ which contributes to $S\left(U_{1}, \mathcal{O}\right)_{\mathfrak{m}}$. Let $\Pi$ denote the stable base change of $\Sigma$ to $\mathrm{GL}_{n}\left(\mathbb{A}_{E}\right)$, a RACSD automorphic representation. Then $\Pi$ is $\iota$-ordinary, and one of the following holds: 
1. For each $i=0, \ldots, s$, we have $\Pi_{w_{i}} \cong \mathrm{St}_{m, E_{w_{i}}} \boxplus \mathrm{St}_{3, E_{w_{i}}} \boxplus \mathrm{St}_{1, E_{w_{i}}}$.

2. There exists $i \in\{0, \ldots, s\}$ such that $r_{\iota}(\Pi)\left(t_{w_{i}}\right)$ has Jordan decomposition corresponding either to the partition $n=(n-2)+1+1$ or to the trivial partition $n=n$. Moreover, $\Pi$ is cuspidal.

Proof. Let us choose some $i \in\{0, \ldots, s\}$ and now write $w=w_{i}$ to simplify notation. Then $\Pi_{w}$ is semistable, just as in [CT15, Lemma 4.3]. [In fact, the proof of [CT15, Lemma 4.3] should be corrected as follows, starting on line 9 of the proof (notations as in loc. cit.).

Now fix $v=v_{i}, w=w_{i}$ for some $i=0, \ldots, s$. Since $\sigma_{0}=\otimes_{i=0}^{s} \sigma_{0, v_{i}}$ is semi-stable, we can take for $j \neq i$ the function $f_{v_{j}}=\left[\mathfrak{B}_{v_{i}}\right]$ (the characteristic function of $\mathfrak{B}_{v_{i}}$ ). Letting $f_{v}$ vary, we obtain an identity

$$
\sum_{\sigma} c(\sigma)\left\langle\operatorname{tr} \sigma, f_{v}\right\rangle=\left\langle\operatorname{tr} \pi_{w} \times I_{c}, f_{E_{w}}\right\rangle
$$

with $\sigma$ running over the representations of $G\left(F_{v}\right), c(\sigma)$ a positive integer (a multiplicity), and $c\left(\sigma_{0}\right)>0$.

The rest of the argument is correct. Note however that the trace $\left\langle\operatorname{tr} \pi_{N_{0}(E)}^{\text {norm }},\left(\overline{f_{E_{w}}}\right) P_{0}\right\rangle$ should be a twisted trace. We also point out that it should follow from Mok's results, completed by Mœglin [Mœg07], that semi-stable representations are always sent to semi-stable representations by base change.] Since it is in the image of the stable base change map, there exists by [Mok15, Lemma 2.2.1] a partition $n=n_{1}+\cdots+n_{r}$, the even parts occurring twice ("parity condition"), and quadratic unramified characters $\psi_{1}, \ldots, \psi_{r}: E_{w}^{\times} \rightarrow \mathbb{C}^{\times}$ such that $\Pi_{w} \cong \operatorname{St}_{n_{1}, E_{w}}\left(\psi_{1}\right) \boxplus \cdots \boxplus \operatorname{St}_{n_{r}, E_{w}}\left(\psi_{r}\right)$.

We have $\overline{r_{\iota}(\Pi)} \cong \bar{\rho}_{1} \oplus \bar{\rho}_{2}$, by the Chebotarev density theorem. We consider the natural (closure) ordering on conjugacy classes of nilpotent $n \times n$ matrices (see e.g. [Tay08, §2]). If a nilpotent $N \in M_{n}(\mathcal{O})$ specializes to $\bar{N} \in M_{n}(k)$, we have

$$
\operatorname{dim}_{K} \operatorname{ker}\left(N^{j}\right) \leq \operatorname{dim}_{k} \operatorname{ker}\left(\bar{N}^{j}\right)
$$

We can choose, after possibly enlarging $K$, a continuous homomorphism $r: G_{E} \rightarrow \operatorname{GL}_{n}(\mathcal{O})$, together with an isomorphism $r \otimes_{\mathcal{O}} \overline{\mathbb{Q}}_{l} \cong r_{\iota}(\Pi)$, such that the reduction $r \bmod \lambda$ is semi-simple. Let $N=r\left(t_{w}\right)-1$. The above inequality shows that $\left(n_{1}, \ldots, n_{r}\right) \geq(m, 3,1)$ in the partial ordering of partitions corresponding to that of nilpotent conjugacy classes. We see that $r \leq 3$; the parity condition implies that $r=1$ or $r=3$. We find that the possible partitions of length 3 are $(m, 3,1),(m+1,2,1)$ and $(m+2,1,1)$, the middle one being impossible by the parity condition. We must show that in the case $(m, 3,1)$, the characters $\psi_{1}, \psi_{2}$ and $\psi_{3}$ are all trivial.

In this case, the natural map $\left(\operatorname{ker} N^{i}\right) \otimes_{\mathcal{O}} k \rightarrow \operatorname{ker}\left(N^{i} \otimes_{\mathcal{O}} k\right)$ is an isomorphism for each $i \geq 0$. Let $\phi_{w} \in G_{E_{w}}$ denote a lift of Frobenius; then $\phi_{w}$ acts on ker $N^{i}$. Comparing these eigenvalues for the representations $\bar{r}$ and $\bar{\rho}_{1} \oplus \bar{\rho}_{2}$, we see that we must have $\iota^{-1} \psi_{1} \equiv \iota^{-1} \psi_{2} \equiv \iota^{-1} \psi_{3} \equiv 1 \bmod \lambda$. Since these are quadratic characters, they must in fact be trivial.

Finally, $\Pi$ must be cuspidal in case 2 since $\bar{\rho}_{1}, \bar{\rho}_{2}$ are absolutely irreducible. If $\Pi$ was not cuspidal, the associated semi-simple Galois representation $r_{\iota}(\Pi)$ would be reducible with degrees $(m, 4)$ and this is incompatible with the Jordan decomposition.

\subsection{Raising the level}

We now come to the main theorem of this section.

Theorem 4.2. Suppose that $l=5$ or $l=7$. Then there exists an automorphic representation $\Sigma$ of $G\left(\mathbb{A}_{F}\right)$ falling into case 2 of Proposition 4.1.

The remainder of this section is devoted to the proof of this result. We assume from now on that $l=7$, the case $l=5$ being similar (and simpler, given what we have done so far). We also now assume, for contradiction, the following:

$(\star)$ There does not exist an automorphic representation $\Sigma$ of $G\left(\mathbb{A}_{F}\right)$ falling into case 2 of Proposition 4.1. 
In this case, we can partition the representations $\Sigma$ contributing to the space $S\left(U_{1}, \mathcal{O}\right)_{\mathfrak{m}}$ into two types, according to Theorem 3.8 and the discussion following it:

1. The base change $\Pi$ of $\Sigma$ is not cuspidal. In this case, there are exactly $4^{s+1} / 2$ automorphic representations of $G\left(\mathbb{A}_{F}\right)$ with base change $\Pi$, each appearing with multiplicity one.

2. The base change $\Pi$ of $\Sigma$ is cuspidal. In this case, there are exactly $4^{s+1}$ automorphic representations of $G\left(\mathbb{A}_{F}\right)$ with base change $\Pi$, each appearing with multiplicity one.

Choose for each $i=1, \ldots, s$ an Iwahori-spherical element $R_{i}$ of the L-packet $\left\{X_{v_{i}}, Y_{v_{i}}, Z_{v_{i}}, W_{v_{i}}\right\}$ of representations of $G\left(F_{v_{i}}\right)$ with base change $\mathrm{St}_{m, E_{w_{i}}} \boxplus \mathrm{St}_{3, E_{w_{i}}} \boxplus \mathrm{St}_{1, E_{w_{i}}}$, so that $\prod_{i=1}^{s} \varepsilon_{v_{i}}\left(R_{i}\right)=-1$. Let $\mathcal{M}$ denote the intersection, as $i=1, \ldots, s$, of the $R_{i}^{\mathfrak{B}_{v_{i}}}$-isotypic parts of $S\left(U_{1}, \mathcal{O}\right)_{\mathfrak{m}}$. By Theorem 3.8 and Corollary 2.8,

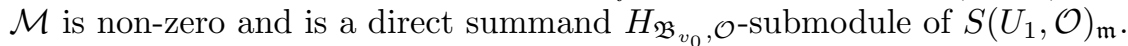

We define a perfect pairing $\langle\cdot, \cdot\rangle: S\left(U_{1}, \mathcal{O}\right) \times S\left(U_{1}, \mathcal{O}\right) \rightarrow \mathcal{O}$ by the formula

$$
\langle f, g\rangle=\sum_{x \in G(F) \backslash G\left(\mathbb{A}_{F}^{\infty}\right) / U_{1}} f(x) g(x) .
$$

Since the group $U_{1}$ is sufficiently small, the groups $G(F) \cap g U_{1} g^{-1}\left(g \in G\left(\mathbb{A}_{F}^{\infty}\right)\right)$, a priori finite, are trivial. A calculation which is essentially the same as the one in [Tay89, p. 271] then shows that this pairing satisfies the formula $\langle a f, g\rangle=\langle f, \jmath(a) g\rangle$ for all $a \in H_{\mathfrak{B}_{v_{0}}, \mathcal{O}}$ and $f, g \in S\left(U_{1}, \mathcal{O}\right), \jmath: H_{\mathfrak{B}_{v_{0}}, \mathcal{O}} \rightarrow H_{\mathfrak{B}_{v_{0}}, \mathcal{O}}$ being the canonical anti-involution.

Proposition 4.3. 1. The above pairing induces perfect pairings

$$
\langle\cdot, \cdot\rangle_{\mathfrak{m}}: S\left(U_{1}, \mathcal{O}\right)_{\mathfrak{m}} \times S\left(U_{1}, \mathcal{O}\right)_{\mathfrak{m}} \vee \rightarrow \mathcal{O} .
$$

and

$$
\langle\cdot, \cdot\rangle_{\mathfrak{m}}: S\left(U_{1}, k\right)_{\mathfrak{m}} \times S\left(U_{1}, k\right)_{\mathfrak{m} \vee} \rightarrow k
$$

2. The above pairing vanishes on restriction to the subspace $S\left(U_{0}, k\right)_{\mathfrak{m}} \times S\left(U_{0}, k\right)_{\mathfrak{m}} \vee$.

3. The above pairing induces a perfect pairing $\mathcal{M} \times \mathcal{N} \rightarrow \mathcal{O}$. The induced perfect duality between $\mathcal{M}_{\mathcal{O}}$ $k \times \mathcal{N} \otimes_{\mathcal{O}} k$ vanishes on restriction to the subspace $\mathcal{M}^{U_{0}} \otimes_{\mathcal{O}} k \times \mathcal{N}^{U_{0}} \otimes_{\mathcal{O}} k$.

Proof. The proof is the same as that of [CT15, Proposition 4.5].

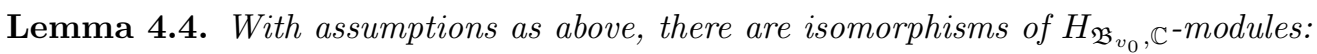

$$
\mathcal{M} \otimes_{\mathcal{O}, \iota} \mathbb{C} \cong\left(X_{v_{0}}^{\mathfrak{B}_{v_{0}}}\right)^{a} \oplus\left(Y_{v_{0}}^{\mathfrak{B}_{v_{0}}}\right)^{b} \oplus\left(Z_{v_{0}}^{\mathfrak{B}_{v_{0}}}\right)^{c} \cong \mathcal{N} \otimes_{\mathcal{O}, \iota} \mathbb{C}
$$

where $a>b=c \geq 0$.

Proof. Since the spaces $\mathcal{M}$ and $\mathcal{N}$ are dual, it is enough to prove the assertion for $\mathcal{M}$. By Theorem 3.8, there exist finite sets $S^{\text {cusp }}, S^{\text {end }}$ of automorphic representations $\Pi$ of $\mathrm{GL}_{n}\left(\mathbb{A}_{E}\right)$, finite-dimensional and non-zero $\mathbb{C}$-vector spaces $V_{\Pi}$ for each $\Pi \in S^{\text {cusp }} \cup S^{\text {end }}$, and a decomposition of $H_{\mathfrak{B}_{v_{0}}}, \mathbb{C}$-modules:

$$
\mathcal{M} \otimes_{\mathcal{O}, \iota} \mathbb{C} \cong\left[\bigoplus_{\Pi \in S^{\text {cusp }}} V_{\Pi} \otimes_{\mathbb{C}}\left(X_{v_{0}}^{\mathfrak{B}_{v_{0}}} \oplus Y_{v_{0}}^{\mathfrak{B}_{v_{0}}} \oplus Z_{v_{0}}^{\mathfrak{B}_{v_{0}}}\right)\right] \oplus\left[\bigoplus_{\Pi \in S^{\text {end }}} V_{\Pi} \otimes_{\mathbb{C}} X_{v_{0}}^{\mathfrak{B}_{v_{0}}}\right] .
$$

Moreover, $S^{\text {end }}$ is non-empty (apply Theorem 3.8 to our initial datum $\pi_{1} \boxplus \pi_{2}$ ). It follows that we can take $a=\sum_{\Pi \in S^{\text {cusp }}} \operatorname{dim} V_{\Pi}+\sum_{\Pi \in S^{\text {end }}} \operatorname{dim} V_{\Pi}$, and $b=c=\sum_{\Pi \in S^{\text {cusp }}} \operatorname{dim} V_{\Pi}$. 
We recall that $H_{\mathfrak{B}_{v_{0}}, \mathcal{O}}$ has an abelian subalgebra $\mathcal{O}[\Lambda]$. Let $\chi: \mathcal{O}[\Lambda] \rightarrow \mathcal{O}$ be the character giving the action of $\mathcal{O}[\Lambda]$ on $X_{v_{0}}^{\mathfrak{B}_{v_{0}}} \otimes_{\mathbb{C}, l^{-1}} \overline{\mathbb{Q}}_{l}$, and let $\bar{\chi}: \mathcal{O}[\Lambda] \rightarrow k$ be its reduction modulo $l$. There is an induced perfect duality $\mathcal{M}(\bar{\chi}) \times \mathcal{N}(J \bar{\chi}) \rightarrow \mathcal{O}$, where we write, as in $\S 2.3,(?)(\bar{\chi})$ for the localization at ker $\bar{\chi} \subset \mathcal{O}[\Lambda]$.

Let us write $\mathcal{M}_{1} \subset \mathcal{M}$ and $\mathcal{N}_{1} \subset \mathcal{N}$ for the $\mathfrak{P}_{v_{0}}$-fixed vectors inside the spaces $\mathcal{M}(\bar{\chi})$ and $\mathcal{N}(j \bar{\chi})$, respectively. These are direct summands as $\mathcal{O}$-modules, of rank equal to $a+b$, by Proposition 2.9. On the other hand, Theorem 2.5 shows that $X_{v_{0}}$ has one exponent congruent mod $l$ to $[2,1,0,-1], Y_{v_{0}}$ has three, and $Z_{v_{0}}$ none, since $q \equiv-1 \bmod l$. Thus $\mathcal{M}(\bar{\chi})$ and $\mathcal{N}(\jmath \bar{\chi})$ are of rank $a+3 b$.

Remark 4.5. The reader can check that this argument fails for $l>7$. In particular, the space $Z_{v_{0}}(\bar{\chi})$ is no longer trivial.

Since the perfect duality between $\mathcal{M}(\bar{\chi}) \otimes_{\mathcal{O}} k$ and $\mathcal{N}(\jmath \bar{\chi}) \otimes_{\mathcal{O}} k$ vanishes on restriction to the subspace $\mathcal{M}_{1} \otimes_{\mathcal{O}} k \times \mathcal{N}_{1} \otimes_{\mathcal{O}} k$, we must have $2(a+b) \leq a+3 b$, hence $a \leq b$. This contradicts the assertion of Lemma 4.4 that $a>b$. This contradiction implies that our assumption $(\star)$ above must be incorrect, and completes the proof of Theorem 4.2 .

\section{Level-raising and Galois theory}

In this section, which is essentially self-contained, we prove a level-raising result, using Galois-theoretic arguments. The need for this result is explained at the beginning of $\S 4$. We start with a residual representation $\bar{\rho}=\bar{\rho}_{1} \oplus \bar{\rho}_{2}$ which is a sum of two irreducible pieces, say of dimensions $n_{1}, n_{2}$. We accomplish level-raising when there is an automorphic lift of $\bar{\rho}$ which locally has a Weil-Deligne representation $(r, N)$ such that $N$ has a Jordan block of dimension strictly greater than $\sup \left(n_{1}, n_{2}\right)$. Any such lift is automatically irreducible. This tension between lifts which are reducible and lifts which have prescribed ramification locally allows us to exploit the geometric techniques developed in [Tho15] to pass to another automorphic lift which has regular nilpotent ramification locally at the given place.

In this way we prove Theorem 5.1. In order to streamline its later application, we then state and prove Theorem 5.7, which essentially combines Theorem 5.1 with the main result of [Tho15]. This result will then be applied, together with the work done in $\S 4$, to deduce the automorphy of symmetric power Galois representations in $\S 6$.

\subsection{A level-raising theorem}

Let $E$ be an imaginary $\mathrm{CM}$ field with maximal totally real subfield $F$. Fix a prime $l$ and an isomorphism $\iota: \overline{\mathbb{Q}}_{l} \cong \mathbb{C}$. Fix an integer $n \geq 2$ and a RACSDC automorphic representation $\Pi$ of $\mathrm{GL}_{n}\left(\mathbb{A}_{E}\right)$ satisfying the following hypotheses:

1. $\overline{r_{\iota}(\Pi)}=\bar{\rho}_{1} \oplus \bar{\rho}_{2}$, where $\operatorname{dim} \bar{\rho}_{i}=n_{i}$ and the $\bar{\rho}_{i}$ are pairwise non-isomorphic, absolutely irreducible, and each satisfy $\bar{\rho}_{i}^{c} \cong \bar{\rho}_{i}^{\vee} \epsilon^{1-n}$. We assume that $n_{1}>n_{2}$ and that $\overline{r_{\iota}(\Pi)}$ is primitive, i.e. not induced from a proper extension of $E$.

2. There exists a place $w_{0}$ of $E$ such that $\Pi_{w_{0}}$ has an Iwahori-fixed vector and, writing $\operatorname{rec}_{E_{w_{0}}}^{T}\left(\Pi_{w_{0}}\right)=$ $(r, N), N$ has a Jordan block of length strictly greater than $n_{1}$. We write $v_{0}$ for the place of $F$ below $w_{0}$.

The purpose of this section is to prove the following theorem.

Theorem 5.1. With notation as above, suppose that the following further hypotheses are in effect:

1. $\Pi$ is ı-ordinary.

2. $l>3$ and $l$ is coprime to each of $n_{1}, n_{2}$ and $n$. The product $n_{1} n_{2}$ is even.

3. Each $\left.\bar{\rho}_{i}\right|_{G_{E\left(\zeta_{l}\right)}}$ is adequate, and $E\left(\zeta_{l}\right)$ is not contained in the fixed field of ad $\overline{r_{\iota}(\Pi)}$. 
4. There exists a $C M$ extension $E_{0} / E$ linearly disjoint from the extension of $E\left(\zeta_{l}\right)$ cut out by $\bar{\rho}$, and RAECSDC automorphic representations $\left(\pi_{1}, \chi_{1}\right)$ and $\left(\pi_{2}, \chi_{2}\right)$ of $\mathrm{GL}_{n_{1}}\left(\mathbb{A}_{E_{0}}\right)$ and $\mathrm{GL}_{n_{2}}\left(\mathbb{A}_{E_{0}}\right)$, respectively, such that $\pi_{1}, \pi_{2}$ are $\iota$-ordinary, and $\frac{r_{\iota}\left(\pi_{i}\right)}{r_{i}} \bar{\rho}_{G_{E_{0}}}$ for $i=1,2$.

Then there exists a soluble $C M$ extension $E_{1}$ of $E$, linearly disjoint from the extension of $E\left(\zeta_{l}\right)$ cut out by $\overline{r_{\iota}(\Pi)}$, and a RACSDC automorphic representation $\Pi_{1}$ of $\mathrm{GL}_{n}\left(\mathbb{A}_{E_{1}}\right)$ satisfying the following conditions:

1. $\Pi_{1}$ is ı-ordinary.

2. $\left.\overline{r_{\iota}\left(\Pi_{1}\right)} \cong \overline{r_{\iota}(\Pi)}\right|_{G_{E_{1}}}$.

3. There exists a place $w_{1}$ of $E_{1}$ dividing $w_{0}$ such that $\Pi_{1, w_{1}}$ is an unramified twist of the Steinberg representation.

The proof will make heavy use of the techniques of the paper [Tho15], to which we make frequent references. After making a preliminary soluble base change, we can assume that the following further hypotheses hold:

1. There exists a regular algebraic, conjugate self-dual and $\iota$-ordinary automorphic representation $\Pi^{\prime}$ of $\mathrm{GL}_{n}\left(\mathbb{A}_{E}\right)$ which is everywhere unramified, and satisfies $\overline{r_{\iota}\left(\Pi^{\prime}\right)} \cong \overline{r_{\iota}(\Pi)}$. ( $\Pi^{\prime}$ can be constructed using an easy generalization of [CHT08, Lemma 4.4.1].)

2. Every place of $F$ dividing $l$ or above which $\Pi$ is ramified splits in $E$. For each place $v \mid l$ of $F$, the local degree $\left[F_{v}: \mathbb{Q}_{l}\right]$ is even.

3. The extension $E / F$ is everywhere unramified, and $\Pi$ is everywhere unipotently ramified.

4. If $w$ is a place of $E$ dividing $l$ or at which $\Pi$ is ramified, then $\left.\overline{r_{\iota}(\Pi)}\right|_{G_{E_{w}}}$ is trivial.

5. For each place $w \mid l$ of $E$, we have $\left[E_{w}: \mathbb{Q}_{l}\right]>n(n+1) / 2+1$. For each place $w \nmid l$ of $E$ at which $\Pi$ is ramified, the highest power of $l$ dividing $q_{v}-1$ is strictly greater than $n$.

In this case $[F: \mathbb{Q}]$ is even and there exists a unitary group $G$ in $n$ variables over $F$ satisfying the following properties:

1. $G$ is split by $E$, and is quasi-split at every finite place.

2. $G\left(F_{\infty}\right)$ is compact.

Choose a finite set $V_{0}$ of finite places of $E$ with the following properties:

1. If $M / E$ is a Galois extension of $E$ contained in the extension of $E\left(\zeta_{l}\right)$ cut out by $\overline{r_{\iota}(\Pi)}$ and $\operatorname{Gal}(M / E)$ is simple, then there exists a place of $V_{0}$ which does not split in $M$.

2. $V_{0}$ contains no place of $E$ which divides $l$ or above which $\Pi$ is ramified.

We write $S_{l}$ for the set of places of $F$ above $l$. Let $S_{a}$ denote a finite, non-empty set of places of $F$, split in $E$ and coprime to $V_{0}$, such that if $w$ is a place of $E$ above an element of $S_{a}$, then $\Pi_{w}$ is unramified, ad $\overline{r_{\iota}(\Pi)}\left(\right.$ Frob $\left._{w}\right)$ is trivial and $q_{w} \not \equiv 1 \bmod l$. (Such places exist because of the assumption on ad $\overline{r_{\iota}(\Pi)}$.) Let $T$ denote the set of places of $F$ below a place at which $\Pi$ is ramified, together with $S_{l} \cup S_{a}$. Thus by definition, $T$ is coprime to $V_{0}$ and every place of $T$ splits in $E$. We choose for each place $v \in T$ a place $\widetilde{v}$ of $E$ dividing it, and write $\widetilde{T}$ for the set of these places. We define $\widetilde{S}_{l}=\left\{\widetilde{v} \mid v \in S_{l}\right\}$ and $\widetilde{S}_{a}=\left\{\widetilde{v} \mid v \in S_{a}\right\}$.

We say that a soluble CM extension $L / E$ is good if every place of $V_{0} \cup \widetilde{S}_{a}$ is split in $L$. Given such an extension, we write $L^{+}$for the maximal totally real subfield of $L$ and $S_{l, L}, S_{a, L}$ and $T_{L}$ for the sets of places of $L^{+}$dividing $S_{l}, S_{a}$ and $T$, respectively. If $v \in T_{L}$ then there is a unique place $\widetilde{v}$ of $L$ dividing $v$ and extending $\widetilde{\left.v\right|_{F}}$. If $S \subset T_{L}$ is any set of places then we define $\widetilde{S}=\{\widetilde{v} \mid v \in S\}$, as above.

We fix an integral model of the group $G$ over $\mathcal{O}_{F}$ exactly as in [CT15, §3.3], as well as identifications $\iota_{\widetilde{v}}: G\left(F_{v}\right) \cong \mathrm{GL}_{n}\left(E_{\widetilde{v}}\right)$ for each $v \in T$. After base extension to $\mathcal{O}_{L}, L$ a good extension, we also obtain identifications $\iota_{\widetilde{v}}: G\left(L_{v}^{+}\right) \cong \mathrm{GL}_{n}\left(L_{\widetilde{v}}\right)$ for any $v \in T_{L}$. 
We fix a finite extension $K \subset \overline{\mathbb{Q}}_{l}$ of $\mathbb{Q}_{l}$. We write $\mathcal{O} \subset K$ for the ring of integers, $\lambda \subset \mathcal{O}$ for the maximal ideal, and $k=\mathcal{O} / \lambda$ for the residue field. If $v \in T_{L}$, we will consider the following local deformation problems, in the sense of [Tho15, Definition 3.6]:

1. If $v \in S_{l, L}$, then there is the ordinary lifting $\operatorname{ring} R_{v}^{\triangle}$ defined in [Tho15, §3.3.2], following Geraghty [Ger].

2. If $v \in S_{a, L}$ then we consider the unrestricted local deformation problem, represented by the ring $R_{v}^{\square}$. With assumptions as above, $R_{v}^{\square}$ is formally smooth over $\mathcal{O}$, the universal lifting being unramified.

3. If $v \nmid l, q_{v} \equiv 1 \bmod l$, and $\chi_{v}=\left(\chi_{v, 1}, \ldots, \chi_{v, n}\right)$ is a choice of $n$-tuple of characters $\chi_{v, i}: \mathcal{O}_{L_{\tilde{r}}}^{\times} \rightarrow \mathcal{O}^{\times}$ which are trivial after reduction modulo $\lambda$, then there is the ring $R_{v}^{\chi_{v}}$ which classifies liftings of $\left.\overline{r_{\iota}(\Pi)}\right|_{G_{L_{\tilde{v}}}}$ whose restriction to inertia has characteristic polynomial $\prod_{i=1}^{n}\left(X-\chi_{v, i}\right)$, as defined in [Tho15, §3.3.3]. In the special case that the characters $\chi_{v, i}$ are all trivial, we write $R_{v}^{\chi v}=R_{v}^{1}$.

4. If $v \nmid l$, and $q_{v} \equiv 1 \bmod l$, then there is the $\operatorname{ring} R_{v}^{\mathrm{St}}$ classifying unipotently ramified lifts for which a Frobenius lift has eigenvalues $\alpha, q_{v} \alpha, \ldots, q_{v}^{n-1} \alpha$ for some $\alpha$, as defined in [Tho15, §3.3.4].

5. If $v \nmid l$ and $q_{v} \equiv 1 \bmod l$, then we define a new local deformation problem as follows. Let us write $\mathbf{m}$ for a choice of partition $n=m_{1}+\cdots+m_{k}, m_{1} \geq m_{2} \cdots \geq m_{k}$. The local deformation ring $R_{v}^{\mathbf{m}}$ is the maximal $\mathcal{O}$-flat reduced quotient of $R_{v}^{1}$ classifying lifts for which a lift of Frob $_{\widetilde{v}}^{-1}$ has characteristic polynomial in the scheme $\operatorname{Pol}_{n}\left(\mathbf{m}, q_{v}\right)$ of [Tay08, §2]. Note that if $\mathbf{m}$ is the partition $n=n$, then $R_{v}^{\mathbf{m}}=R_{v}^{\mathrm{St}}$ by definition. (We can specify a local deformation problem by specifying a local deformation ring, thanks to [BLGHT11, Lemma 3.2].)

Lemma 5.2. Let $x \in \operatorname{Spec} R_{v}^{\mathbf{m}}[1 / l]$ be a closed point induced by a homomorphism $\rho: G_{L_{\tilde{v}}} \rightarrow \mathrm{GL}_{n}(\mathcal{O})$ such that $\rho \otimes_{\mathcal{O}} \overline{\mathbb{Q}}_{l}$ is pure, in the sense of [TY0\%, Lemma 1.4]. Then Spec $R_{v}^{1}[1 / l]$ is formally smooth over $K$ at $x$, and there is a unique minimal prime $Q_{v}$ of $R_{v}^{1}$ in the kernel of the induced homomorphism $R_{v}^{1} \rightarrow \mathcal{O}$. Moreover, $Q_{v}$ contains the kernel of the homomorphism $R_{v}^{1} \rightarrow R_{v}^{\mathbf{m}}$.

Proof. We first note that the underlying reduced ring of $R_{v}^{1}$ is $\mathcal{O}$-flat and equidimensional of dimension $1+n^{2}$, cf. [Tay08, Proposition 3.1] and [Tho12, Lemma 3.15]. On the other hand, writing $R_{v}^{\square}$ for the unrestricted lifting ring of which $R_{v}^{1}$ is a quotient, one knows (cf. [BLGGT14, Lemma 1.3.2]) that Spec $R_{v}^{\square}[1 / l]$ is equidimensional of dimension $n^{2}$, and formally smooth over $K$ at those closed points corresponding to pure representations. It follows that there is a unique irreducible component of $\operatorname{Spec} R_{v}^{1}[1 / l]$ passing through each closed point $x$ as in the statement of the lemma, which is formally smooth over $K$ at $x$.

Let us write $Q_{v} \subset R_{v}^{1}$ for the corresponding minimal prime; it remains to show that $Q_{v}$ contains the kernel of the homomorphism $R_{v}^{1} \rightarrow R_{v}^{\mathbf{m}}$. This follows from the proof of [Tho12, Lemma 3.15]. Indeed, in the notation at the bottom of [Tho12, p. 865], $x$ defines a $K$-point of $\mathcal{M}(\mathbf{m})$ (in fact, of $\mathcal{M}(\mathbf{m})^{0}$ ) which is contained in no other irreducible component of $\mathcal{M}$. The inclusion $\mathcal{M}(\mathbf{m}) \subset \mathcal{M}(\mathbf{m})^{\prime}$ now implies the result.

Recall the disconnected group $\mathcal{G}_{n}$ over $\mathbb{Z}$ classifying representations of unitary type considered in [CHT08, §2.1], [Tho15, §3.1]. There exists an extension of $\overline{r_{\iota}(\Pi)}$ to a homomorphism $\bar{r}: G_{F} \rightarrow \mathcal{G}_{n}(k)$, which is necessarily Schur in the sense of [Tho15, Definition 3.2] and satisfies $\nu \circ \bar{r}(c)=-1$. After possibly enlarging $K$, we can also choose a lift of $\bar{r}$ to a homomorphism $r: G_{F} \rightarrow \mathcal{G}_{n}(\mathcal{O})$ such that $\left.r\right|_{G_{E}} \otimes_{\mathcal{O}} \overline{\mathbb{Q}}_{l} \cong r_{\iota}(\Pi)$. Suppose given a good extension $L / E$, a finite set $R$ of places of $L^{+}$dividing $T \backslash\left(S_{l} \cup S_{a}\right)$, and for each $v \in R$ a local lifting ring $R_{v}$ of one of the types $3-5$ defined above. In terms of the pair $\mathcal{D}=\left(L,\left\{R_{v}\right\}_{v \in R}\right)$ we define:

1. The Iwasawa algebra $\Lambda_{L}=\widehat{\otimes}_{v \in S_{l, L}} \Lambda_{v}$, where $\left.\Lambda_{v}=\mathcal{O} \llbracket\left(I_{L_{\tilde{v}}}(l)\right)^{a b}\right)^{n} \rrbracket$.

2. A global deformation problem in the sense of [Tho15, §3.2], $\mathcal{S}\left(\left\{R_{v}\right\}_{v \in R}\right)=$

$$
\left(L / L^{+}, R \cup S_{l, L} \cup S_{a, L}, \widetilde{R} \cup \widetilde{S}_{l, L} \cup \widetilde{S}_{a, L}, \Lambda_{L}, \bar{r}, \epsilon^{1-n} \delta_{L / L^{+}}^{n},\left\{R_{v}^{\triangle}\right\}_{v \in S_{l, L}} \cup\left\{R_{v}^{\square}\right\}_{v \in S_{a, L}} \cup\left\{R_{v}\right\}_{v \in R}\right) .
$$

3. An open compact subgroup $U=\prod_{v} U_{v} \subset G\left(\mathbb{A}_{L^{+}}^{l, \infty}\right)$ defined as follows: 
(a) For $v$ inert in $L, U_{v} \subset G\left(L_{v}^{+}\right)$is a hyperspecial maximal compact subgroup.

(b) For $v \notin R \cup S_{l, L} \cup S_{a, L}$ split in $L, U_{v}=G\left(\mathcal{O}_{L_{v}^{+}}\right)$.

(c) For $v \in S_{a, L}, U_{v}=\iota_{\widetilde{v}}^{-1} \operatorname{ker}\left(\mathrm{GL}_{n}\left(\mathcal{O}_{L_{\tilde{v}}}\right) \rightarrow \mathrm{GL}_{n}(k(\widetilde{v}))\right)$.

(d) For $v \in R, U_{v}=\iota_{\widetilde{v}}^{-1} \operatorname{Iw}(\widetilde{v})$.

Then for any open compact subgroup $U_{l} \subset G\left(L_{l}^{+}\right), U \cdot U_{l}$ is sufficiently small, as the set $S_{a}$ is non-empty.

4. A space of modular forms $S_{\mathcal{D}}$, which is the space denoted $S_{\chi}\left(U\left(\mathfrak{l}^{\infty}\right), K / \mathcal{O}\right)^{\vee}$ in [Tho15, Definition 4.2]. The datum $\chi=\left\{\chi_{v}\right\}_{v \in R}$ here is taken to consist of the corresponding character $\chi_{v}$ for those local deformation problems $R_{v}$ such that $R_{v}=R_{v}^{\chi_{v}}$, and to be the trivial character otherwise. This is a finite free $\Lambda_{L}$-module (as the level is sufficiently small, cf. [Tho15, Proposition 4.3]).

5. A Hecke algebra $\mathbb{T}_{\mathcal{D}}$, which is the localization of the algebra denoted $\mathbb{T}_{\chi}^{T_{L}}\left(U\left(\mathfrak{l}^{\infty}\right), \mathcal{O}\right)$ in $[$ Tho15, Definition 4.2] (with the same choice of $\chi$ as in the previous point) at the maximal ideal corresponding to a descent of the representation $\Pi^{\prime}$ to $G$ with non-zero $U^{l}$-fixed vectors. (This descent exists, by [CT14, Proposition 2.9], and the maximal ideal is independent of this choice.)

We refer to a pair $\left(L,\left\{R_{v}\right\}_{v \in R}\right)$ satisfying the above conditions as a deformation datum. Associated to a choice of deformation datum is the global deformation ring $R_{\mathcal{D}}=R_{\mathcal{S}\left(\left\{R_{v}\right\}_{v \in R}\right)}^{\text {univ }}$ (notation as in [Tho15, Proposition 3.8]) and its subring the universal pseudodeformation ring $P_{\mathcal{D}}=P_{\mathcal{S}\left(\left\{R_{v}\right\}_{v \in R}\right)}$ (notation as in [Tho15, Definition 3.27]). The inclusion $P_{\mathcal{D}} \subset R_{\mathcal{D}}$ is a finite homomorphism of Noetherian $\Lambda_{L}$-algebras ([Tho15, Proposition 3.29]). If for each $v \in R \backslash\left(S_{l, L} \cup S_{a, L}\right), R_{v}=R_{v}^{\chi_{v}}$ for some choice of $\chi_{v}$, then there is a surjective homomorphism $P_{\mathcal{D}} \rightarrow \mathbb{T}_{\mathcal{D}}$ of $\Lambda_{L}$-algebras. (The proof of this assertion follows similar lines to that of [Tho15, Proposition 4.12].) In this case we write $I_{\mathcal{D}}$ for the kernel of this algebra homomorphism, and $J_{\mathcal{D}}=I_{\mathcal{D}} R_{\mathcal{D}}$. If $\mathcal{D}$ is any deformation datum, then we write $R_{\mathcal{D}}^{\text {red }}$ for the quotient of $R_{\mathcal{D}}$ which classifies reducible deformations ([Tho15, Definition 3.31]). Whenever it is defined, $\mathbb{T}_{\mathcal{D}}$ is a finite torsion-free $\Lambda_{L^{-}}$ algebra ([Tho15, Proposition 4.3]). Whenever $R_{v}=R_{v}^{\chi v}$ or $R_{v}^{\mathrm{St}}$ for each $v \in R$, every minimal prime of $R_{\mathcal{D}}$ has dimension at least $\operatorname{dim} \Lambda_{L}=1+n\left[L^{+}: \mathbb{Q}\right]$ ([Tho15, Proposition 3.9]).

Lemma 5.3. Fix a partition $\mathbf{m}$ with $m_{1}>n_{1}=\operatorname{dim} \overline{\rho_{1}}$. Let $\mathcal{D}=\left(L,\left\{R_{v}\right\}_{v \in R_{0}} \cup\left\{R_{v}^{\mathbf{m}}\right\}_{v \in R_{1}}\right)$ be a deformation datum. Let $\Delta$ denote the Galois group of the maximal abelian l-extension of $L$ unramified outside $l$, and let $\Delta_{0}$ denote the Galois group of the maximal abelian l-extension of $L$ unramified outside $l$ in which every prime of $\widetilde{R}_{1} \cup \widetilde{R}_{1}^{c}$ splits completely. Let

$$
d=\operatorname{dim}_{\mathbb{Q}_{l}} \operatorname{ker}\left(\Delta \otimes_{\mathbb{Z}_{l}} \mathbb{Q}_{l} \rightarrow \Delta_{0} \otimes_{\mathbb{Z}_{l}} \mathbb{Q}_{l}\right)^{c=-1} .
$$

Then $\operatorname{dim} R_{\mathcal{D}}^{\text {red }} \leq 1+n\left[L^{+}: \mathbb{Q}\right]-d$, and $R_{\mathcal{D}}^{\text {red }}$ is a finite $\Lambda_{L}$-algebra.

Proof. It suffices to show that for each minimal prime $Q \subset R_{\mathcal{D}}^{\text {red }}$, we have $\operatorname{dim} R_{\mathcal{D}}^{\text {red }} / Q \leq 1+n\left[L^{+}: \mathbb{Q}\right]-d$ and $R_{\mathcal{D}}^{\text {red }} / Q$ is a finite $\Lambda_{L}$-algebra. Let us now fix such a prime and write $C=R_{\mathcal{D}}^{\text {red }} / Q$. We may write $\bar{r}$ as a sum of homomorphisms $\bar{r}_{i}: G_{F} \rightarrow \mathcal{G}_{n_{i}}(k)$, as in [Tho15, §3.5]. For $i=1,2$, we define $\Lambda_{i, L}=\otimes_{v \in S_{l, L}} \Lambda_{i, v}$ and $\Lambda_{i, v}=\mathcal{O} \llbracket I_{L_{\tilde{v}}}(l)^{n_{i}} \rrbracket$. We introduce the auxiliary deformation problems

$$
\begin{gathered}
\mathcal{S}_{1}=\left(L / L^{+}, S_{l, L} \cup S_{a, L} \cup R_{0} \cup R_{1}, \widetilde{S}_{l, L} \cup \widetilde{S}_{a, L} \cup \widetilde{R}_{0} \cup \widetilde{R}_{1},\right. \\
\left.\Lambda_{1, L}, \bar{r}_{1}, \epsilon^{1-n} \delta_{L / L^{+}}^{n},\left\{R_{v}^{\triangle}\right\}_{v \in S_{l, L}} \cup\left\{R_{v}^{\square}\right\}_{v \in S_{a}} \cup\left\{R_{v}^{1}\right\}_{v \in R_{0} \cup R_{1}}\right), \\
\mathcal{S}_{2}=\left(L / L^{+}, S_{l, L} \cup S_{a, L} \cup R_{0} \cup R_{1}, \widetilde{S}_{l, L} \cup \widetilde{S}_{a, L} \cup \widetilde{R}_{0} \cup \widetilde{R}_{1},\right. \\
\left.\Lambda_{2, L}, \bar{r}_{2}, \epsilon^{1-n} \delta_{L / L^{+}}^{n},\left\{R_{v}^{\triangle}\right\}_{v \in S_{l, L}} \cup\left\{R_{v}^{\square}\right\}_{v \in S_{a}} \cup\left\{R_{v}^{1}\right\}_{v \in R_{0} \cup R_{1}}\right) .
\end{gathered}
$$

(We have abused notation slightly here by writing $R_{v}^{\triangle}, R_{v}^{\square}, R_{v}^{1}$ also for the local lifting rings corresponding to the local representations $\left.\bar{r}_{1}\right|_{G_{L_{\tilde{v}}}}$ and $\left.\bar{r}_{2}\right|_{G_{L_{\tilde{v}}}}$ of dimensions $n_{1}$ and $n_{2}$, respectively.) Arguing as in the proof of [Tho15, Theorem 7.1], one can show the following: 
1. Let $r_{\mathcal{D}}^{\text {red }} \bmod Q=r_{1} \oplus r_{2}: G_{L^{+}} \rightarrow \mathcal{G}_{n}(C)$ denote the reduction $\bmod Q$ of the universal deformation. Then for each $v \in S_{l, L}$ there exists an isomorphism $\Lambda_{1, L} \widehat{\otimes}_{\mathcal{O}} \Lambda_{2, L} \cong \Lambda_{L}$ such that, with the induced structures on $C$ of $\Lambda_{1, L^{-}}$and $\Lambda_{2, L^{-}}$algebra, $r_{1}$ is a deformation of type $\mathcal{S}_{1}$ and $r_{2}$ is a deformation of type $\mathcal{S}_{2}$.

2. For each $i=1,2$, the universal deformation $\operatorname{ring} R_{\mathcal{S}_{i}}^{\text {univ }}$ is finite over $\Lambda_{i, L}$ of dimension $1+n_{i}\left[L^{+}: \mathbb{Q}\right]$.

3. The induced map $R_{\mathcal{S}_{1}}^{\text {univ }} \widehat{\otimes}_{\mathcal{O}} R_{\mathcal{S}_{2}}^{\text {univ }} \rightarrow C$ is surjective.

(This is where we use the assumption in Theorem 5.1 that each of $\bar{\rho}_{1}, \bar{\rho}_{2}$ are potentially ordinarily automorphic.) It follows that $C$ is a finite $\Lambda_{L}$-algebra. We now bound the dimension of $C$. For each $i=1,2$, let $\psi_{i}: G_{L} \rightarrow \mathcal{O}^{\times}$be the Teichmüller lift of $\left.\operatorname{det} \bar{r}_{i}\right|_{G_{L}}$. Write $R_{\mathcal{S}_{i}, \psi_{i}}^{\text {univ }}$ for the quotient of $R_{\mathcal{S}_{i}}^{\text {univ }}$ classifying deformations with determinant $\psi_{i}$. According to [Tho15, Lemma 3.36], there are isomorphisms $R_{\mathcal{S}_{i}}^{\text {univ }} \cong R_{\mathcal{S}_{i}, \psi_{i}}^{\text {univ }} \widehat{\otimes}_{\mathcal{O}} \mathcal{O} \llbracket \Delta /(c+1) \rrbracket$, and hence

$$
R_{\mathcal{S}_{1}}^{\text {univ }} \widehat{\otimes}_{\mathcal{O}} R_{\mathcal{S}_{2}}^{\text {univ }} / \lambda \cong R_{\mathcal{S}_{1}, \psi_{1}}^{\text {univ }} / \lambda \widehat{\otimes}_{k} R_{\mathcal{S}_{2}, \psi_{2}}^{\text {univ }} / \lambda \widehat{\otimes}_{k} k \llbracket \Delta /(c+1) \rrbracket \widehat{\otimes}_{k} k \llbracket \Delta /(c+1) \rrbracket .
$$

Let

$$
\Psi_{1}, \Psi_{2}: \Delta /(c+1) \rightarrow\left(k \llbracket \Delta /(c+1) \rrbracket \widehat{\otimes}_{k} k \llbracket \Delta /(c+1) \rrbracket\right)^{\times}
$$

denote the universal characters valued in this ring. With the above identification, the universal deformation has the form $\left(r_{\mathcal{S}_{1}, \psi_{1}}^{\text {univ }} \otimes \Psi_{1}\right) \oplus\left(r_{\mathcal{S}_{2}, \psi_{2}}^{\text {univ }} \otimes \Psi_{2}\right)$. (Recall that $n_{1}$ and $n_{2}$ are coprime to $l$.) Let $A=R_{\mathcal{S}_{1}, \psi_{1}}^{\text {univ }} \widehat{\otimes}_{\mathcal{O}} R_{\mathcal{S}_{2}, \psi_{2}}^{\text {univ }}$, with maximal ideal $\mathfrak{m}_{A}$. We have (by [Mat89, Theorem 15.1]) $\operatorname{dim} C \leq \operatorname{dim} A+\operatorname{dim} C / \mathfrak{m}_{A} C$, and $C / \mathfrak{m}_{A} C$ is a quotient of $B=k \llbracket \Delta /(c+1) \rrbracket \widehat{\otimes}_{k} k \llbracket \Delta /(c+1) \rrbracket$. In fact, we have $\operatorname{dim} C / \mathfrak{m}_{A} C \leq \operatorname{dim} B / J$, where $B / J$ is the quotient of $B$ classifying pairs $\Psi_{1}, \Psi_{2}$ such that $\left.\left[\left(\bar{r}_{1} \otimes \Psi_{1}\right) \oplus\left(\bar{r}_{2} \otimes \Psi_{2}\right)\right]\right|_{G_{L_{\tilde{v}}}}$ is of type $R_{v}^{\mathrm{m}}$ for each $v \in R_{1}$. We now show that $\operatorname{dim} B / J \leq \operatorname{dim} B-d$, which will complete the proof.

Let $P \subset B$ be a prime such that $\left.\left[\left(\bar{r}_{1} \otimes \Psi_{1}\right) \oplus\left(\bar{r}_{2} \otimes \Psi_{2}\right)\right]\right|_{G_{L_{\tilde{v}}}} \bmod P$ is of type $R_{v}^{\mathbf{m}}$, for each $v \in R_{1}$. Let $\phi_{v}$ be a Frobenius lift at $\widetilde{v}$. Then $\left[\left(\bar{r}_{1} \otimes \Psi_{1}\right) \oplus\left(\bar{r}_{2} \otimes \Psi_{2}\right)\right]\left(\phi_{v}\right)$ has an eigenvalue of multiplicity $m_{1}>n_{1}>n_{2}$; since its eigenvalues are visibly either distinct of multiplicities $n_{1}+n_{2}=n$ or equal, we must be in the latter case, and so $\Psi_{1}\left(\phi_{v}\right)=\Psi_{2}\left(\phi_{v}\right)$. Since $P$ was arbitrary, we see that $\Psi_{1}\left(\right.$ Frob $\left._{\widetilde{v}}\right)=\Psi_{2}\left(\right.$ Frob $\left._{\widetilde{v}}\right)$ modulo the nilradical of $J$, for each $v \in R_{1}$. The result now follows from the definition of $d$.

Lemma 5.4. Let $\mathcal{D}=\left(L,\left\{R_{v}^{\chi_{v}}\right\}_{v \in R_{0}} \cup\left\{R_{v}^{\mathbf{m}}\right\}_{v \in R_{1}}\right)$ be a deformation datum, where $\mathbf{m}$ is the partition of $n$ corresponding to the Jordan decomposition of $N, \operatorname{rec}_{E_{w_{0}}}^{T}\left(\Pi_{w_{0}}\right)=(r, N)$, and $R_{1}$ consists of places of $L^{+}$ dividing $v_{0}$. Let $\mathcal{D}_{0}=\left(L,\left\{R_{v}^{\chi_{v}}\right\}_{v \in R_{0}} \cup\left\{R_{v}^{1}\right\}_{v \in R_{1}}\right)$. (Thus there is a surjection $R_{\mathcal{D}_{0}} \rightarrow R_{\mathcal{D}}$ of Noetherian $\Lambda_{L}$-algebras.) Then $\operatorname{dim} R_{\mathcal{D}} / J_{\mathcal{D}_{0}} R_{\mathcal{D}}=\operatorname{dim} \Lambda_{L}$.

Proof. We use the properties of the Hecke algebra described in the paragraph preceding Lemma 5.3. The ring $R_{\mathcal{D}_{0}} / J_{\mathcal{D}_{0}} R_{\mathcal{D}_{0}}$ is a finite $\mathbb{T}_{\mathcal{D}_{0}}$-algebra, hence a finite $\Lambda_{L}$-algebra. It follows that $\operatorname{dim} R_{\mathcal{D}} / J_{\mathcal{D}_{0}} R_{\mathcal{D}} \leq \operatorname{dim} \Lambda_{L}$. To prove the lemma, we must therefore show that $\operatorname{dim} R_{\mathcal{D}} / J_{\mathcal{D}_{0}} R_{\mathcal{D}} \geq \operatorname{dim} \Lambda_{L}$. The existence of the base change $\Pi_{L}$ implies the existence of a homomorphism (after possibly enlarging $K$ ) $R_{\mathcal{D}} \rightarrow \mathcal{O}$ whose kernel contains $J_{\mathcal{D}_{0}} R_{\mathcal{D}}$. Let $r: G_{L^{+}} \rightarrow \mathcal{G}_{n}(\mathcal{O})$ denote a representative of the corresponding deformation. (Thus we have $\left.r\right|_{G_{L}} \otimes_{\mathcal{O}} \overline{\mathbb{Q}}_{l} \cong r_{\iota}\left(\Pi_{L}\right)$.)

For each $v \in R_{1}$, let $J_{v}=\operatorname{ker}\left(R_{v}^{1} \rightarrow R_{v}^{\mathbf{m}}\right)$, and let $Q_{v}$ denote the unique minimal prime in the kernel of the homomorphism $R_{v}^{1} \rightarrow \mathcal{O}$ induced by $\left.r\right|_{G_{L_{v}}}$, cf. Lemma 5.2. Thus $J_{v} \subset Q_{v}$. Let $Q_{0} \subset \mathbb{T}_{\mathcal{D}_{0}}$ denote a minimal prime in the kernel of the map $\mathbb{T}_{\mathcal{D}_{0}} \rightarrow \mathcal{O}$ induced by $\iota^{-1} \Pi_{L}^{\infty}$, and let $Q \subset R_{\mathcal{D}_{0}}$ be a prime of dimension $\operatorname{dim} \Lambda_{L}=\operatorname{dim} \mathbb{T}_{\mathcal{D}_{0}} / Q_{0}$, minimal over $Q_{0} R_{\mathcal{D}_{0}}$, and contained in the kernel of the map $R_{\mathcal{D}_{0}} \rightarrow \mathcal{O}$.

We show that $Q$ contains the kernel of the surjective homomorphism $R_{\mathcal{D}_{0}} \rightarrow R_{\mathcal{D}}$. Since this kernel is generated by the ideals $J_{v}, v \in R_{1}$, it suffices to show that for each $v \in R_{1}$, we have $J_{v} \subset Q$ or even $Q_{v} \subset Q$. However, if $Q_{v}^{\prime} \subset R_{v}^{1}$ is a minimal prime in the kernel of the homomorphism $R_{v}^{1} \rightarrow R_{\mathcal{D}_{0}} / Q$ then it is contained in the kernel of the homomorphism $R_{v}^{1} \rightarrow R_{\mathcal{D}_{0}} \rightarrow \mathcal{O}$, hence equal to $Q_{v}$. The result follows.

Lemma 5.5. Let $\mathcal{D}_{0}^{\chi}=\left(L_{0},\left\{R_{v}^{\chi_{v}}\right\}_{v \in R}\right)$ be a deformation datum. Suppose that for each $v \in R$, the characters $\chi_{v, 1}, \ldots, \chi_{v, n}$ are pairwise distinct. Let $L$ be a good extension containing $L_{0}$ and such that for each place 
$\widetilde{w}$ of $L$ dividing a place $\widetilde{v}$ of $L_{0}, v \in R$, the restriction $\left.\chi_{v, i}\right|_{I_{L_{\tilde{w}}}}$ is trivial. Let $\mathcal{D}=(L, \emptyset)$. Then there is a commutative diagram

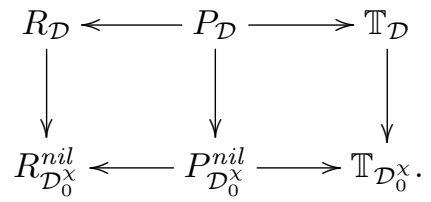

(Here we write $A^{\text {nil }}$ for the quotient of $A$ by its nilradical, the superscript $A^{\text {red }}$ already being reserved for reducible deformations.)

Proof. It is easy to see that the morphism $R_{\mathcal{D}} \rightarrow R_{\mathcal{D}_{0}^{x}}^{\text {nil }}$ exists provided that for each $v \in R$ and each place $\widetilde{w}$ of $L$ dividing $\widetilde{v}$, the universal lifting $\rho: G_{L_{0, \tilde{v}}} \rightarrow \mathrm{GL}_{n}\left(R_{v}^{\chi_{v}}\right.$,nil $)$ becomes unramified on restriction to $G_{L_{\tilde{w}}}$. Since $R_{v}^{\chi v}$,nil is a domain ([Tho12, Proposition 3.16]), this can be checked after extending scalars to $\mathcal{K}=\operatorname{Frac}\left(R_{v}^{\chi_{v}, \text { nil }}\right)$, a field of characteristic zero. Let $\sigma \in G_{L_{0, \tilde{v}}}$ denote a generator of tame inertia. The element $\rho(\sigma) \in \mathrm{GL}_{n}(\mathcal{K})$ is regular semi-simple, because its characteristic polynomial $\prod_{i=1}^{n}\left(X-\chi_{v, i}(\sigma)\right)$ has distinct roots, by hypothesis. If $N \geq 1$ is an integer such that $\rho\left(\sigma^{N}\right)$ is unipotent, it must therefore be trivial. The existence of the left hand commutative square above is now immediate.

For the right hand square, we must construct the arrow $\mathbb{T}_{\mathcal{D}} \rightarrow \mathbb{T}_{\mathcal{D}_{0}^{\chi}}$. (We remark that these Hecke algebras are reduced.) As in the proof of [Tho15, Proposition 4.18], this can be reduced to the existence of soluble base change and descent for the unitary group $G$, in the guise of [CT14, Proposition 2.9]. Our appeal to loc. cit. is the reason for our assumption at the beginning of this section that $n_{1}$ and $n_{2}$ are distinct, and that $n_{1} n_{2}$ is even.

Lemma 5.6. Suppose there exists a deformation datum $\mathcal{D}=(L, \emptyset)$ and a prime $\mathfrak{p} \subset R_{\mathcal{D}}$ of dimension 1 and characteristic $l$ satisfying the following conditions:

1. Let $A$ denote the normalization of the quotient ring $R_{\mathcal{D}} / \mathfrak{p}$. Then the induced representation $r_{\mathfrak{p}}: G_{L} \rightarrow$ $\mathrm{GL}_{n}(\operatorname{Frac} A)$ is absolutely irreducible.

2. For each $v \in S_{l, L}$, the characters $\psi_{1}^{v}, \ldots, \psi_{n}^{v}: I_{L_{\widetilde{v}}} \rightarrow A^{\times}$induced by the homomorphism $\Lambda_{L} \rightarrow A$ are pairwise distinct.

3. There exists a place $v \in S_{l, L}$ and an element $\sigma \in I_{L_{\tilde{v}}}$ such that the eigenvalues of $r_{\mathfrak{p}}(\sigma)$ satisfy no non-trivial $\mathbb{Z}$-linear relation.

4. The prime $\mathfrak{p}$ contains $J_{\mathcal{D}}$.

5. There exists a place $u \in T_{L} \backslash\left(S_{l, L} \cup S_{a, L}\right)$ such that $\left.r_{\mathfrak{p}}\right|_{G_{L_{\tilde{u}}}}$ is trivial.

Then there exists a RACSDC automorphic representation $\Pi_{1}$ of $\mathrm{GL}_{n}\left(\mathbb{A}_{L}\right)$ satisfying the following conditions:

1. $\Pi_{1}$ is ı-ordinary.

2. $\left.\overline{r_{\iota}\left(\Pi_{1}\right)} \cong \overline{r_{\iota}(\Pi)}\right|_{G_{L}}$.

3. $\Pi_{1, \widetilde{u}}$ is an unramified twist of the Steinberg representation.

Proof. We can assume that if $\mathfrak{q}=P_{\mathcal{D}} \cap \mathfrak{p}$, then $\operatorname{Frac}\left(R_{\mathcal{D}} / \mathfrak{p}\right)=\operatorname{Frac}\left(P_{\mathcal{D}} / \mathfrak{q}\right)$; otherwise, replace $\mathfrak{p}$ by a suitable twist as in [Tho15, Corollary 4.14].

We introduce the auxiliary deformation data $\mathcal{D}^{1}=\left(L,\left\{R_{u}^{1}\right\}\right)$ and $\mathcal{D}^{\mathrm{St}}=\left(L,\left\{R_{u}^{\mathrm{St}}\right\}\right)$. Thus $R_{\mathcal{D}^{\mathrm{St}}}$ is naturally a quotient of $R_{\mathcal{D}^{1}}$. To prove the lemma, it suffices to show that there is a minimal prime $Q \subset R_{\mathcal{D}^{\text {St }}}$ such that $R_{\mathcal{D}^{\text {St }}} / Q$ is a finite $\Lambda_{L}$-algebra, and $J_{\mathcal{D}^{1}} R_{\mathcal{D}^{\text {St }}} \subset Q$. Indeed, we also have $\operatorname{dim} R_{\mathcal{D}^{\text {St }}} / Q \geq \operatorname{dim} \Lambda_{L}$ (see the paragraph before Lemma 5.3), so $Q$ lies above a minimal prime of $\Lambda_{L}$, and then a classicality result in Hida theory (e.g. [Ger, Lemma 2.6.4]) implies the existence of a characteristic zero point of $R_{\mathcal{D}^{\text {St }}}$ which arises from an automorphic representation with the desired properties. 
To show the existence of such a minimal prime, we appeal to an $R=\mathbb{T}$ theorem proved in [Tho15]. Let $Q \subset R_{\mathcal{D}^{\text {St }}}$ be a minimal prime inside $\mathfrak{p}$, and let $Q^{1} \subset R_{\mathcal{D}^{1}}$ be minimal inside $Q$. Then [Tho15, Corollary 4.20] implies that $J_{\mathcal{D}^{1}} \subset Q^{1}$ and $R_{\mathcal{D}^{1}} / Q^{1}$ is a finite $\Lambda_{L}$-algebra of dimension $\operatorname{dim} \Lambda_{L}$. It follows that the natural map $R_{\mathcal{D}^{1}} / Q^{1} \rightarrow R_{\mathcal{D}^{\text {st }}} / Q$ is an isomorphism.

To complete the proof it remains to justify the application of [Tho15, Corollary 4.20], or in other words to check that the hypotheses (1)-(5) of [Tho15, Theorem 4.19] hold. In the notation there, we take $R=\{u\}$ and $S(B)=\emptyset$. By [Tho15, Corollary 5.7], hypothesis (1) of [Tho15, Theorem 4.19] is satisfied when the conditions enumerated at the beginning of [Tho15, §5.2] are satisfied. It is easy to see that these conditions hold in the case considered here. Hypothesis (2) of [Tho15, Theorem 4.19] is satisfied here by assumption. Hypotheses (3) and (4) are satisfied because of the preliminary reductions immediately after the statement of Theorem 5.1. Hypothesis (5) is satisfied by the first paragraph of the current proof. We note that it is assumed in the proof of [Tho15, Theorem 4.19] that the set $S(B)$ is non-empty; however, this assumption plays no role in the proof.

We can now complete the proof of Theorem 5.1. By Lemma 5.6, it suffices to find a deformation datum $\mathcal{D}=(L, \emptyset)$ and a prime $\mathfrak{p} \subset R_{\mathcal{D}}$ satisfying the hypotheses of Lemma 5.6. Arguing as in the proof of [Tho15, Theorem 7.1], we can find a deformation datum $\mathcal{D}_{0}=\left(L_{0},\left\{R_{v}^{\mathbf{m}}\right\}_{v \in R}\right)$ satisfying the following conditions:

- $R$ consists of the set of places of $L_{0}^{+}$dividing $v_{0}$ and $\mathbf{m}$ is the partition of $n$ corresponding to the Jordan decomposition of $N$, where $\operatorname{rec}_{E_{\widetilde{v}_{0}}^{T}}^{T}\left(\Pi_{\widetilde{v}_{0}}\right)=(r, N)$.

- For each place $v \mid l$ of $L_{0}^{+},\left[L_{0, v}^{+}: \mathbb{Q}_{l}\right]>n^{2}$.

- The integer $d=d_{\mathcal{D}_{0}}$ of Lemma 5.3 satisfies $d>n^{2}+1$.

We introduce also the deformation datum $\mathcal{D}_{0}^{\chi}=\left(L_{0},\left\{R_{v}^{\chi v}\right\}_{v \in R}\right)$. Lemma 5.3 then implies that $\operatorname{dim} R_{\mathcal{D}_{0}}^{\text {red }} / \lambda<$ $n\left[L_{0}^{+}: \mathbb{Q}\right]-n^{2}$.

Let $u$ be a place of $L_{0}$ above $v_{0}$. By Lemma $5.4, R_{\mathcal{D}_{0}} / J_{\mathcal{D}_{0}^{1}} R_{\mathcal{D}_{0}}$ is a finite $\Lambda_{L_{0}}$-algebra of dimension $\operatorname{dim} \Lambda_{L_{0}}=1+n\left[L_{0}^{+}: \mathbb{Q}\right]$, hence $\operatorname{dim} R_{\mathcal{D}_{0}} /\left(J_{\mathcal{D}_{0}^{1}}, \lambda\right) \geq n\left[L_{0}^{+}: \mathbb{Q}\right]$, hence $\operatorname{dim} R_{\mathcal{D}_{0}} /\left(J_{\mathcal{D}_{0}^{1}}, \lambda, \mathfrak{m}_{R_{u}^{1}}\right) \geq n\left[L_{0}^{+}:\right.$ $\mathbb{Q}]-n^{2}>\operatorname{dim} R_{\mathcal{D}_{0}}^{\text {red }} / \lambda$. We can therefore choose a dimension 1 prime $\mathfrak{p}_{0} \subset R_{\mathcal{D}_{0}} /\left(J_{\mathcal{D}_{0}^{1}}, \lambda, \mathfrak{m}_{R_{u}^{1}}\right)$ which does not come from $R_{\mathcal{D}_{0}}^{\text {red }} / \lambda$, and this prime will satisfy conditions 1 , 4, and 5 of Lemma 5.6. Conditions 2 and 3 can be ensured by choosing $\mathfrak{p}_{0}$ also to avoid countably many quotients of $R_{\mathcal{D}_{0}} /\left(J_{\mathcal{D}_{0}^{1}}, \lambda, \mathfrak{m}_{R_{u}^{1}}\right)$ which have dimension bounded above by $\sup _{v \mid l}\left(n\left[L_{0}^{+}: \mathbb{Q}\right]-\left[L_{0, v}^{+}: \mathbb{Q}_{l}\right]\right)<n\left[L_{0}^{+}: \mathbb{Q}\right]-n^{2}$. The possibility of finding a dimension 1 prime missing these countably many quotients is guaranteed by [Tho15, Lemma 1.9]. These quotients are described explicitly in [Tho15, p. 861, Proof of Theorem 6.1], where [Tho15, Lemma 1.9] is employed for the same purpose. Having chosen a prime $\mathfrak{p}_{0}$ satisfying conditions $1-5$ of the lemma, we identify it with its pullback to a prime of $R_{\mathcal{D}_{0}^{1}}$.

The rings $R_{\mathcal{D}_{0}^{\chi}} / \lambda$ are canonically identified as $\chi$ varies. Similarly, the modules $S_{\mathcal{D}_{0}^{\chi}} / \lambda$ are canonically identified as $\chi$ varies; cf. [Ger, Lemma 2.2.6]. It follows that a prime $\mathfrak{p}^{\chi} \subset R_{\mathcal{D}_{0}^{\chi}} / \lambda$ contains $J_{\mathcal{D}_{0}^{\chi}}$ if and only if the corresponding prime $\mathfrak{p}^{1} \subset R_{\mathcal{D}_{0}^{1}} / \lambda$ contains $J_{\mathcal{D}_{0}^{1}}$. Fix a choice of $\chi$ such that for each $v \in R$, the characters $\chi_{v, 1}, \ldots, \chi_{v, n}$ are pairwise distinct, and let $\mathfrak{p}^{\chi} \subset R_{\mathcal{D}_{0}^{\chi}} / \lambda$ be the prime corresponding in this way to $\mathfrak{p}_{0}$.

By Lemma 5.5, we can find a good extension $L$ and a deformation datum $\mathcal{D}=(L, \emptyset)$ such that $L$ contains $L_{0}$, the places in $\widetilde{S}_{l, L}$ all split in $L_{0}$, and there exists a commutative diagram

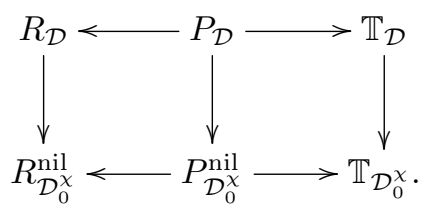

We let $\mathfrak{p}=\operatorname{ker}\left(R_{\mathcal{D}} \rightarrow R_{\mathcal{D}_{0}^{\chi}} / \mathfrak{p}^{\chi}\right) \subset R_{\mathcal{D}}$. The existence of the above diagram implies that $J_{\mathcal{D}} \subset \mathfrak{p}$, so that hypothesis 4 of Lemma 5.6 holds. The representation $r_{\mathfrak{p}}=\left.r_{\mathfrak{p}_{0}}\right|_{G_{L}}$ is irreducible because $r_{\mathfrak{p}_{0}}$ is primitive and $r_{\mathfrak{p}}$ is multiplicity-free (because of the existence of $\sigma$ ), so hypothesis 1 holds. The other hypotheses hold by construction. This completes the proof. 


\subsection{An automorphy lifting theorem}

We now use Theorem 5.1 to improve the main theorem of [Tho15]. Let $E$ be an imaginary CM field with maximal totally real subfield $F$. Fix a prime $l$ and an isomorphism $\iota: \overline{\mathbb{Q}}_{l} \cong \mathbb{C}$.

Theorem 5.7. Let $\rho: G_{E} \rightarrow \mathrm{GL}_{n}\left(\overline{\mathbb{Q}}_{l}\right)$ be a continuous representation satisfying the following hypotheses.

1. $\rho^{c} \cong \rho^{\vee} \epsilon^{1-n}$.

2. $\rho$ is ramified at only finitely many places.

3. $\rho$ is ordinary of weight $\lambda$, for some $\lambda \in\left(\mathbb{Z}_{+}^{n}\right)^{\operatorname{Hom}\left(E, \overline{\mathbb{Q}}_{l}\right)}$, in the sense of [Tho15, Definition 2.5].

4. $l>3$ and $E\left(\zeta_{l}\right)$ is not contained in the extension of $E$ cut out by ad $\bar{\rho}$.

5. $\bar{\rho} \cong \bar{\rho}_{1} \oplus \bar{\rho}_{2}$, where the $\left.\bar{\rho}_{i}\right|_{G_{E\left(\zeta_{l}\right)}}$ are adequate, in the sense of [Tho12, §2]. Writing $n_{i}=\operatorname{dim} \bar{\rho}_{i}$, we have $n_{1} \neq n_{2}, n_{1} n_{2}$ is even, and $l$ does not divide $n_{1} n_{2}\left(n_{1}+n_{2}\right)$. The representation $\bar{\rho}$ is primitive.

6. There exists a place $w_{0}$ of $E$, prime to $l$, such that $\rho_{G_{E_{w_{0}}}}^{s s} \cong \oplus_{i=1}^{n} \psi \epsilon^{n-i}$ for some unramified character $\psi: G_{E_{w_{0}}} \rightarrow \overline{\mathbb{Q}}_{l}^{\times}$.

7. There exists a RACSDC automorphic representation $\Pi$ of $\mathrm{GL}_{n}\left(\mathbb{A}_{E}\right)$ such that:

(a) П is ı-ordinary.

(b) $\overline{r_{\iota}(\Pi)} \cong \bar{\rho}$.

(c) Let $\operatorname{rec}_{E_{w_{0}}}^{T}\left(\Pi_{w_{0}}\right)=(r, N)$. Then $N$ has a Jordan block of length strictly greater than $\max \left(n_{1}, n_{2}\right)$.

8. There exists a CM extension $E_{0} / E$ linearly disjoint from the extension of $E\left(\zeta_{l}\right)$ cut out by $\bar{\rho}$, and RAECSDC automorphic representations $\left(\pi_{1}, \chi_{1}\right)$ and $\left(\pi_{2}, \chi_{2}\right)$ of $\mathrm{GL}_{n_{1}}\left(\mathbb{A}_{E_{0}}\right)$ and $\mathrm{GL}_{n_{2}}\left(\mathbb{A}_{E_{0}}\right)$, respectively, such that $\pi_{1}, \pi_{2}$ are $\iota$-ordinary, and $\left.\overline{r_{\iota}\left(\pi_{i}\right)} \cong \bar{\rho}_{i}\right|_{G_{E_{0}}}$ for $i=1,2$.

Then $\rho$ is automorphic: there exists a RACSDC automorphic representation $\pi$ of $\mathrm{GL}_{n}\left(\mathbb{A}_{E}\right)$ such that $\rho \cong$ $r_{\iota}(\pi)$.

Proof. There exists a soluble extension $L / E$ such that the hypotheses of Theorem 5.1 apply to $\Pi_{L}$; thus we can find a further soluble extension $E_{1} / E$, disjoint from the extension of $E\left(\zeta_{l}\right)$ cut out by $\bar{\rho}$, and a RACSDC automorphic representation $\Pi_{1}$ of $\mathrm{GL}_{n}\left(\mathbb{A}_{E_{1}}\right)$ satisfying the hypotheses of [Tho15, Theorem 7.1 ] with respect to $\left.\rho\right|_{E_{1}}$. This implies that $\left.\rho\right|_{E_{1}}$ is automorphic, and irreducible by property (3) in Theorem 5.1. It follows by soluble descent that $\rho$ is itself automorphic.

\section{Symmetric power functoriality}

In this section we use what we have done so far to draw consequences for the existence of symmetric power functoriality for $\mathrm{GL}_{2}\left(\mathbb{A}_{F}\right)$.

Theorem 6.1. Let $F$ be a totally real field, and let $(\pi, \chi)$ be a RAESDC automorphic representation of $\mathrm{GL}_{2}\left(\mathbb{A}_{F}\right)$ which is not automorphically induced from a quadratic $C M$ extension. Then:

1. Suppose that $F \cap \mathbb{Q}\left(\zeta_{5}\right)=\mathbb{Q}$. Then the $6^{\text {th }}$ symmetric power lifting of $\pi$ exists, as a cuspidal automorphic representation of $\mathrm{GL}_{7}\left(\mathbb{A}_{F}\right)$.

2. Suppose that $F \cap \mathbb{Q}\left(\zeta_{7}\right)=\mathbb{Q}$. Then the $8^{\text {th }}$ symmetric power lifting of $\pi$ exists, as a cuspidal automorphic representation of $\mathrm{GL}_{9}\left(\mathbb{A}_{F}\right)$.

By a series of reductions which are essentially the same as those of [CT15, 6$]$, it is enough to prove the following result. 
Theorem 6.2. Let $l \in\{5,7\}$, and let $F$ be a totally real number field. Fix an isomorphism $\iota: \overline{\mathbb{Q}}_{l} \cong \mathbb{C}$. Let $(\pi, \chi)$ be a RAESDC automorphic representation of $\mathrm{GL}_{2}\left(\mathbb{A}_{F}\right)$, and suppose that the following hypotheses hold:

1. $\pi$ is ı-ordinary of weight zero, i.e. at every infinite place $\tau: F \hookrightarrow \mathbb{R}$, the infinity type of $\pi$ is $a_{\tau}=$ $(1 / 2,-1 / 2)$.

2. Let $\rho=r_{\iota}(\pi)$. Then the residual representation $\bar{\rho}: G_{F} \rightarrow \mathrm{GL}_{2}\left(\overline{\mathbb{F}}_{l}\right)$ is irreducible, and its image contains a conjugate of $\mathrm{SL}_{2}\left(\mathbb{F}_{l^{a}}\right)$ for some a $>1$. Moreover, writing $\varphi \in \operatorname{Gal}\left(\overline{\mathbb{Q}}_{l} / \mathbb{Q}_{l}\right)$ for a lift of the arithmetic Frobenius, the representations $\left.(\varphi \bar{\rho} \otimes \bar{\rho})\right|_{G_{F\left(\zeta_{l}\right)}}$ and $\left.\left(\operatorname{Sym}^{l-3} \bar{\rho}\right)\right|_{G_{F\left(\zeta_{l}\right)}}$ are adequate.

3. There exists a place $u_{0}$ of $F$, coprime to 2, such that $\pi_{u_{0}}$ is an unramified twist of the Steinberg representation and $q_{u_{0}}$ is a primitive root mod $l$. Moreover, $\bar{\rho}$ is ramified at $u_{0}$.

Then $\operatorname{Sym}^{l+1} \rho$ is automorphic.

Proof. Let $L / F$ be the extension of $F\left(\zeta_{l}\right)$ cut out by $\bar{\rho}$, and let $S$ be a finite set of finite places of $F$ with the property that for any intermediate extension $L / L_{0} / F$ which is Galois over $F$ with simple Galois group, there exists a place of $S$ which is not split in $L_{0}$. We assume moreover that $S$ is disjoint from the set of places of $F$ which divide $l$ or at which $\rho$ is ramified.

Let $F_{0} / F$ be an $S$-split totally real quadratic extension in which $u_{0}$ is inert, and let $\omega_{F_{0} / F}: G_{F} \rightarrow \overline{\mathbb{Q}}_{l}^{\times}$ denote the corresponding quadratic character. Let $E / F$ be a CM imaginary quadratic extension which is $S$-split, ramified at $u_{0}$, and in which every place of $F$ dividing $l$ splits. By [CHT08, Lemma 4.1.4], we can find a finite order character $\psi: G_{E} \rightarrow \overline{\mathbb{Q}}_{l}^{\times}$, unramified above $u_{0}$, such that $\psi \psi^{c}=\left.r_{\iota}(\chi)\right|_{G_{E}}$.

Let $F_{1} / F$ be a soluble totally real extension satisfying the following conditions:

1. $F_{1}$ is $S \cup\left\{u_{0}\right\}$-split.

2. For each place $v \mid l$ of $F_{1}$, the local degree $\left[F_{1, v}: \mathbb{Q}_{l}\right]$ is even.

3. The extension $F_{1} \cdot F_{0} / F_{1}$ is everywhere unramified.

4. Let $E_{1}=E \cdot F_{1}$. Then if $w \nmid u_{0}$ is a place of $E_{1}$ at which $\pi_{E_{1}}$ or $\left.\psi\right|_{G_{E_{1}}}$ is ramified, then $w$ is split over $F_{1}$. The extension $E_{1} / F_{1}$ is unramified away from the places dividing $u_{0}$.

Let $\pi_{1}=\left.\pi_{E_{1}} \otimes \iota \psi^{-1}\right|_{E_{1}}$, a RACSDC automorphic representation of $\mathrm{GL}_{2}\left(\mathbb{A}_{E_{1}}\right)$ which is $\iota$-ordinary of weight zero. (It is cuspidal because of the assumption that $\pi_{u_{0}}$ is an unramified twist of the Steinberg representation; this property is preserved under base change.) After possibly replacing $\pi_{1}$ by $\left.\pi_{1} \otimes \iota \omega_{F_{0} / F}\right|_{G_{E_{1}}}$, we can assume that $\pi_{1, w} \cong \mathrm{St}_{2, E_{1, w}}$ for each place $w \mid u_{0}$ of $E_{1}$.

Let $y_{0}, \ldots, y_{s}$ denote the places of $F_{1}$ dividing $u_{0}$, and let $z_{0}, \ldots, z_{s}$ denote the places of $E_{1}$ dividing $y_{0}, \ldots, y_{s}$, respectively. We observe that, by construction, $q_{y_{i}}$ is a primitive root modulo $l$ for each $i=0, \ldots, s$. Let $b$ denote the least integer such that $q_{y_{0}}^{b} \equiv-1 \bmod l$, and choose a cyclic totally real extension $F_{2} / F_{1}$ of degree $b$ which is $S$-split and in which $y_{0}$ is inert and each place $y_{1}, \ldots, y_{s}$ splits. Let $E_{2}=F_{2} \cdot F_{1}$, and let $\pi_{2}=\pi_{1, E_{2}}$.

Let $\rho_{2}=r_{\iota}\left(\pi_{2}\right)$, and let $\chi_{2}=\operatorname{det} \rho_{2}$. We have an isomorphism of residual representations

$$
\left(\operatorname{Sym}^{l+1} \bar{\rho}_{2}\right)^{\mathrm{ss}} \cong\left({ }^{\varphi} \bar{\rho}_{2} \otimes \bar{\rho}_{2}\right) \oplus \bar{\chi}_{2}^{2} \operatorname{Sym}^{l-3} \bar{\rho}_{2} .
$$

By [CT15, Proposition 3.10] and base change for $U_{2}$, we can find a RACSDC automorphic representation $\pi_{2}^{\prime}$ of $\mathrm{GL}_{2}\left(\mathbb{A}_{E_{2}}\right)$ with the following properties:

1. $\pi_{2}^{\prime}$ is $\iota$-ordinary and for every embedding $\tau: E_{2} \hookrightarrow \overline{\mathbb{Q}}_{l}$, we have $\operatorname{HT}_{\tau}\left(r_{\iota}\left(\pi_{2}^{\prime}\right)\right)=\{(1-l) / 2,(1+l) / 2\}$.

2. For each $i=0, \ldots, s, \pi_{2, z_{i}}^{\prime} \cong \mathrm{St}_{2, E_{2, z_{i}}} \otimes \iota \omega$, where $\omega$ denotes the Teichmüller lift of $\epsilon^{(l-1) / 2}$.

3. $\overline{r_{\iota}\left(\pi_{2}^{\prime}\right)} \cong \epsilon^{(l-1) / 2} \otimes \bar{\rho}_{2}$. 
4. If $\pi_{2}^{\prime}$ is ramified at a place $w \nmid u_{0}$ of $E_{2}$, then $w$ is split over $F_{2}$.

By $\left[\right.$ Ram00, Theorem M], there exists a RAECSDC automorphic representation $\left(\Pi_{1}^{\prime},|\cdot|^{2-l}\right)$ of $\mathrm{GL}_{4}\left(\mathbb{A}_{E_{2}}\right)$ such that $r_{\iota}\left(\Pi_{1}^{\prime}\right) \cong \varphi_{\iota}\left(\pi_{2}^{\prime}\right) \otimes \epsilon^{(1-l) / 2} \otimes r_{\iota}\left(\pi_{2}\right)$. There also exists a RACSDC automorphic representation $\Pi_{2}^{\prime}$ of $\mathrm{GL}_{l-2}\left(\mathbb{A}_{E_{2}}\right)$ such that $r_{\iota}\left(\Pi_{2}^{\prime}\right) \cong \operatorname{Sym}^{l-3} \rho_{2}$, by [GJ78] (if $l=5$ ) and by [Kim03] (if $l=7$ ).

We define $\Pi_{1}=\Pi_{1}^{\prime} \otimes|\cdot|^{(l-2) / 2}$ and $\Pi_{2}=\Pi_{2}^{\prime} \otimes \iota\left(\epsilon \chi_{2}\right)^{2}$. Then $\Pi_{1} \boxplus \Pi_{2}$ is a conjugate self-dual, regular algebraic automorphic representation of $\mathrm{GL}_{l+2}\left(\mathbb{A}_{E_{2}}\right)$ of weight $\boldsymbol{\lambda}=0$, and satisfies the following conditions:

1. $\overline{r_{\iota}\left(\Pi_{1} \boxplus \Pi_{2}\right)} \cong\left(\varphi^{\varphi} \bar{\rho}_{2} \otimes \bar{\rho}_{2}\right) \oplus \bar{\chi}_{2}^{2} \operatorname{Sym}^{l-3} \bar{\rho}_{2}$.

2. $\Pi_{1} \boxplus \Pi_{2}$ is $\iota$-ordinary (by [CT14, Lemma 2.6]).

3. For each $i=0, \ldots, s, \Pi_{1, w_{i}} \cong \operatorname{St}_{3, E_{2, w_{i}}}(\omega) \boxplus \operatorname{St}_{1, E_{2, w_{i}}}(\omega)$.

4. For each $i=0, \ldots, s, \Pi_{2, w_{i}} \cong \mathrm{St}_{l-2, E_{2, w_{i}}}$.

In particular, the hypotheses of $\S 4$ apply to $\left(\left.\Pi_{1} \otimes \iota \omega_{F_{0} / F}\right|_{G_{E_{2}}}\right) \boxplus \Pi_{2}$. Let $\Pi$ denote the RACSDC automorphic representation of $\mathrm{GL}_{l+2}\left(\mathbb{A}_{E_{2}}\right)$ whose existence is asserted by Theorem 4.2. Let $E_{0}=E_{2} \cdot F_{0}$, and let $\Pi_{0}=$ $\Pi_{E_{0}}$. We have $\overline{r_{\iota}\left(\Pi_{0}\right)} \cong\left(\operatorname{Sym}^{l+1} \bar{\rho}_{2}\right)^{\mathrm{ss}}$, and Theorem 5.7 now implies that $\left.\operatorname{Sym}^{l+1} \rho_{2}\right|_{G_{E_{0}}}$ is automorphic. The desired result now follows by soluble descent, which applies since the representation $\left.\operatorname{Sym}^{l+1} \rho_{2}\right|_{G_{E_{0}}}$ is irreducible.

\section{Complements: the mixed-parity case}

In this section, we show how to extend our results to those cuspidal automorphic representations associated to Hilbert modular forms whose weights are not of constant parity. Recall that if $F$ is a totally real field and $\pi$ is a cuspidal automorphic representation of $\mathrm{GL}_{2}\left(\mathbb{A}_{F}\right)$ such that $\pi_{\infty}$ is essentially square-integrable, then $\pi$ is associated to Hilbert modular forms of weights $k_{v} \geq 2$ at every place $v \mid \infty$ of $F$. Conversely, every such $\pi$ arises in this fashion. The representation $\pi$ is algebraic (up to twist) if and only if it is associated to a Hilbert modular form of weights $k_{v}$ of parity independent of $v \mid \infty$. This condition is vacuous if $F=\mathbb{Q}$, but non-trivial over every other totally real field $F$, as follows from well-known density results (see for example [Clo86]).

Our proofs in this section rely on the observation (which goes back at least to the work of BlasiusRogawski [BR93]) that a representation $\pi$ which does not satisfy this parity condition does in fact become algebraic up to twist after base change to an imaginary CM extension.

Theorem 7.1. Let $F$ be a totally real field, and let $r \geq 1$ be an integer. Suppose that for every RAESDC automorphic representation $(\pi, \chi)$ of $\mathrm{GL}_{2}\left(\mathbb{A}_{F}\right)$ which is not automorphically induced from a quadratic $C M$ extension, there exists a RAESDC automorphic representation $(\Pi, \Xi)$ of $\mathrm{GL}_{r+1}\left(\mathbb{A}_{F}\right)$ such that

$$
\operatorname{Sym}^{r} \operatorname{rec}_{F_{v}} \pi_{v} \cong \operatorname{rec}_{F_{v}} \Pi_{v}
$$

for all places $v \nmid \infty$ of $F$. (Equivalently, for all primes $l$ and for all isomorphisms $\iota: \overline{\mathbb{Q}}_{l} \cong \mathbb{C}$, we have $\operatorname{Sym}^{r} r_{\iota}(\pi) \cong r_{\iota}(\Pi)$.)

Then for every cuspidal automorphic representation $\pi$ of $\mathrm{GL}_{2}\left(\mathbb{A}_{F}\right)$ such that $\pi_{\infty}$ is essentially square integrable and $\pi$ is not automorphically induced from a quadratic CM extension, there exists a cuspidal automorphic representation $\Pi$ of $\mathrm{GL}_{r+1}\left(\mathbb{A}_{F}\right)$ such that

$$
\operatorname{Sym}^{r} \operatorname{rec}_{F_{v}} \pi_{v} \cong \operatorname{rec}_{F_{v}} \Pi_{v}
$$

for all places $v \nmid \infty$ of $F$. In particular, $L\left(\operatorname{Sym}^{r} \pi, s\right)=L(\Pi, s)$ has an analytic continuation to the entire complex plane, and satisfies the expected functional equation.

Corollary 7.2. Let $F$ be a totally real field, and let $r \geq 1$ be an integer. Let $\pi$ be a cuspidal automorphic representation of $\mathrm{GL}_{2}\left(\mathbb{A}_{F}\right)$ such that $\pi_{\infty}$ is essentially square-integrable. Suppose furthermore that $\pi$ is 
not automorphically induced from a quadratic CM extension. Then there exists a cuspidal automorphic representation $\Pi$ of $\mathrm{GL}_{r+1}\left(\mathbb{A}_{F}\right)$ satisfying

$$
\operatorname{Sym}^{r} \operatorname{rec}_{F_{v}} \pi_{v} \cong \operatorname{rec}_{F_{v}} \Pi_{v}
$$

for all places $v \nmid \infty$ in each of the following cases:

1. F arbitrary and $1 \leq r \leq 4$.

2. $F \cap \mathbb{Q}\left(\zeta_{5}\right)=\mathbb{Q}$ and $r \in\{5,6\}$.

3. $F \cap \mathbb{Q}\left(\zeta_{35}\right)=\mathbb{Q}$ and $r=7$.

4. $F \cap \mathbb{Q}\left(\zeta_{7}\right)=\mathbb{Q}$ and $r=8$.

Proof. The first point is implied by known cases of symmetric power functoriality [GJ78], [KS02], [Kim03]. The remaining points follow on combining Theorem 7.1 with Theorem 6.1 (case $r=6,8$ ) and [CT15, Theorem $1.2]$ ( case $r=5,7$ ).

The remainder of this section is devoted to the proof of Theorem 7.1. We begin with some preliminary results.

Theorem 7.3. 1. Let $F$ be a number field, and $\pi$ a cuspidal automorphic representation of $\mathrm{GL}_{2}\left(\mathbb{A}_{F}\right)$. Then $\mathrm{Sym}^{2} \pi$ exists, and the following are equivalent:

(a) $\mathrm{Sym}^{2} \pi$ is cuspidal.

(b) $\pi$ is not automorphically induced from a quadratic extension of $F$.

(c) For all non-trivial Hecke characters $\eta: F^{\times} \backslash \mathbb{A}_{F}^{\times} \rightarrow \mathbb{C}^{\times}$, we have $\pi \neq \pi \otimes \eta$.

2. Let $F$ be a number field, and $\pi$ a cuspidal automorphic representation of $\mathrm{GL}_{2}\left(\mathbb{A}_{F}\right)$. Let $E / F$ be a quadratic extension. Suppose that $\mathrm{Sym}^{2} \pi$ is cuspidal. Then the representations $\pi_{E}$ and $\mathrm{Sym}^{2} \pi_{E}$ are also cuspidal.

Proof. 1. Immediate from [GJ78].

2. Immediate from [AC89, Theorem 3.4.2]. (Note that as $\mathrm{Sym}^{2} \pi$ is a cuspidal automorphic representation of $\mathrm{GL}_{3}\left(\mathbb{A}_{F}\right)$, it cannot be automorphically induced from a quadratic extension.)

Lemma 7.4. Let $F$ be a totally real field, and let $\pi$ be a cuspidal automorphic representation of $\mathrm{GL}_{2}\left(\mathbb{A}_{F}\right)$ such that $\pi_{\infty}$ is essentially square-integrable. Let $E / F$ be an imaginary $C M$ extension such that $\pi_{E}$ is cuspidal. (Such a field always exists; for example, one can choose $E$ to be ramified at a place where $\pi$ is unramified.) Then there exists a continuous character $\chi: E^{\times} \backslash \mathbb{A}_{E}^{\times} \rightarrow \mathbb{C}^{\times}$such that $\pi_{E} \otimes \chi$ is RACSDC.

Proof. Let $\psi: F^{\times} \backslash \mathbb{A}_{F}^{\times} \rightarrow \mathbb{C}^{\times}$denote the central character of $\pi$. Using the fact, due to Chevalley, that the arithmetic subgroups of the unit group of $F$ are congruence subgroups, we can assume (after passing to a twist) that $\psi$ is of finite order. In particular, $\pi$ is unitary. If $v \mid \infty$ is a place of $F$, then the Langlands parameter $\operatorname{rec}_{F_{v}} \pi_{v}$ is equal to the induction $\operatorname{Ind}_{\mathbb{C}^{x}}^{W_{F_{v}}} \varphi$, where $\varphi(z)=(z /|z|)^{p_{v}}$ for some $p_{v} \in \mathbb{Z}_{>0}$. We have $\psi_{v}(-1)=(-1)^{p_{v}+1}$. (We write $|z|=(z \bar{z})^{1 / 2}=|z|_{\mathbb{C}}^{1 / 2}$ for the usual absolute value on $\mathbb{C}$.) If $\chi: E^{\times} \backslash \mathbb{A}_{E}^{\times} \rightarrow \mathbb{C}^{\times}$ is a continuous unitary character of infinity type

$$
\chi_{\tau}(z)=(z /|z|)^{m_{\tau}},
$$

and $\tau: E \hookrightarrow \mathbb{C}$ is an embedding inducing the place $v$ of $F$, then the Langlands parameter $\tau^{-1} \circ \operatorname{rec}_{E_{v}}\left(\pi_{E} \otimes \chi\right)$ is given by the formula:

$$
z \mapsto\left(\begin{array}{cc}
(z /|z|)^{p_{v}+m_{\tau}} & 0 \\
0 & (z /|z|)^{-p_{v}+m_{\tau}}
\end{array}\right) .
$$


Thus $\pi_{E} \otimes \chi$ is regular algebraic if for each $\tau: E \hookrightarrow \mathbb{C}$ as above, $p_{v}+m_{\tau}$ is an odd integer; while $\pi_{E} \otimes \chi$ is conjugate self-dual if and only if $\chi \chi^{c}=\psi^{-1} \circ \mathbb{N}_{E / F}$.

To prove the lemma, we must therefore show the existence of a character satisfying these two conditions. Choose for each place $v \mid \infty$ of $F$ an embedding $\tau_{v}: E \hookrightarrow \mathbb{C}$ inducing the place $v$ of $F$, and define $m_{\tau_{v}}=p_{v}+1, m_{\tau_{v}^{c}}=-\left(p_{v}+1\right)$. Choose a character $\chi_{0}: E^{\times} \backslash \mathbb{A}_{E}^{\times} \rightarrow \mathbb{C}^{\times}$with infinity type

$$
\chi_{0, \tau}(z)=(z /|z|)^{m_{\tau}}
$$

for each embedding $\tau: E \hookrightarrow \mathbb{C}$. Then the character $\chi_{0} \chi_{0}^{c}$ is of finite order; let $\lambda=\left.\chi_{0}\right|_{F \times} \backslash \mathbb{A}_{F}^{\times}$, so that $\chi_{0} \chi_{0}^{c}=\lambda \circ \mathbb{N}_{E / F}$. The character $\lambda: F^{\times} \backslash \mathbb{A}_{F}^{\times} \rightarrow \mathbb{C}^{\times}$is continuous and of finite order, and we have for each place $v \mid \infty$

$$
\lambda_{v}(-1)=\chi_{0, \tau_{v}}(-1)=(-1)^{p_{v}+1} .
$$

In particular, the quantity $\left(\psi_{v} \lambda_{v}\right)(-1)=1$ is independent of the choice of $v \mid \infty$. By [CHT08, Lemma 4.1.4], then, there exists a character $\chi_{1}: E^{\times} \backslash \mathbb{A}_{E}^{\times} \rightarrow \mathbb{C}^{\times}$of finite order such that $\chi_{1} \chi_{1}^{c}=\left(\psi^{-1} \lambda^{-1}\right) \circ \mathbb{N}_{E / F}=$ $\left(\psi^{-1} \circ \mathbb{N}_{E / F}\right)\left(\chi_{0} \chi_{0}^{c}\right)^{-1}$. The character $\chi=\chi_{0} \chi_{1}$ now has the desired properties.

Lemma 7.5. Let $E$ be an imaginary $C M$ field, and $\pi$ a RACSDC automorphic representation of $\mathrm{GL}_{2}\left(\mathbb{A}_{E}\right)$. Suppose that $\mathrm{Sym}^{2} \pi$ is cuspidal. Then there exists a set $\mathcal{L}$ of rational primes of Dirichlet density 1 such that for all $l \in \mathcal{L}$ and for all isomorphisms $\iota: \overline{\mathbb{Q}}_{l} \cong \mathbb{C}$, the representation $\bar{r}_{\iota}(\pi): G_{E} \rightarrow \mathrm{GL}_{2}\left(\overline{\mathbb{F}}_{l}\right)$ is irreducible, and its image contains a conjugate of $\mathrm{SL}_{2}\left(\mathbb{F}_{l}\right)$.

Proof. By [BLGGT14, Theorem 5.5.2], there exists a set $\mathcal{L}$ of rational primes of Dirichlet density 1 such that for all $l \in \mathcal{L}$ and for all isomorphisms $\iota: \overline{\mathbb{Q}}_{l} \cong \mathbb{C}$, the representations $r_{\iota}(\pi): G_{E} \rightarrow \mathrm{GL}_{2}\left(\overline{\mathbb{Q}}_{l}\right)$ and $\operatorname{Sym}^{2} r_{\iota}(\pi): G_{E} \rightarrow \mathrm{GL}_{3}\left(\overline{\mathbb{Q}}_{l}\right)$ are irreducible. Applying [BLGGT14, Proposition 5.3.2] and possibly shrinking $\mathcal{L}$, we can further assume that the representations $\bar{r}_{\iota}(\pi)$ and $\operatorname{Sym}^{2} \bar{r}_{\iota}(\pi)$ are irreducible. Casting out finitely many elements of $\mathcal{L}$, we can also assume that for each $l \in \mathcal{L}, \pi$ and $E$ are unramified above $l$.

To prove the lemma, it therefore suffices (by the classification of finite subgroups of $\mathrm{PGL}_{2}\left(\overline{\mathbb{F}}_{l}\right)$ ) to show that for all but finitely many choices of $l \in \mathcal{L}$, the projective image of $\bar{r}_{\iota}(\pi)$ contains an element of order strictly greater than 5. (Note that the image cannot be contained in the normalizer of a quadratic torus, since $\operatorname{Sym}^{2} \bar{r}_{\iota}(\pi)$ is, by hypothesis, irreducible.) To show this, let $\boldsymbol{\lambda}=\left(\lambda_{\tau, 1}, \lambda_{\tau, 2}\right)_{\tau: E \hookrightarrow \mathbb{C}}$ denote the weight of $\pi$. Let $k=1+\left|\sup _{\tau} \lambda_{\tau, 1}-\inf _{\tau} \lambda_{\tau, 2}\right|$. If $v \mid l$ is a place of $E$, let $S_{v}$ denote the set of embeddings $E_{v} \hookrightarrow \overline{\mathbb{Q}}_{l}$. If $\tau \in S_{v}$, let $\omega_{\tau}: I_{v} \rightarrow \overline{\mathbb{F}}_{l}^{\times}$denote the corresponding fundamental character. If $l>k+2$ is a prime and $\iota: \overline{\mathbb{Q}}_{l} \cong \mathbb{C}$ is an isomorphism, then for each place $v \mid l$ of $E$ there exists a partition $S_{v}=J_{1} \amalg J_{2}$ such that $\left(\left.\bar{r}_{\iota}(\pi)\right|_{I_{v}}\right)^{\mathrm{ss}}$ is a sum of two characters $\alpha_{1}, \alpha_{2}$, given by the formulae

$$
\begin{aligned}
& \alpha_{1}(x)^{-1}=\prod_{\tau \in J_{1}} \omega_{\tau}(x)^{\lambda_{\iota}-1_{\tau, 1}+1} \prod_{\tau \in J_{2}} \omega_{\tau}(x)^{\lambda_{\iota}-1_{\tau, 2}}, \\
& \alpha_{2}(x)^{-1}=\prod_{\tau \in J_{2}} \omega_{\tau}(x)^{\lambda_{\iota}-1_{\tau, 1}+1} \prod_{\tau \in J_{1}} \omega_{\tau}(x)^{\lambda_{\iota}-1_{\tau, 2}} .
\end{aligned}
$$

Indeed, this follows from the fact that $r_{\iota}(\pi)$ is crystalline with Hodge-Tate weights $\operatorname{HT}_{\tau}\left(r_{\iota}(\pi)\right)=\left\{\lambda_{\iota}-1 \tau_{, 1}+\right.$ $\left.1, \lambda_{\iota^{-1} \tau, 2}\right\}$, together with the results of Fontaine-Laffaille [FL82, $\left.\S \S 4-5\right]$. To prove the lemma, it is therefore enough to show that for $l$ sufficiently large, and for all isomorphisms $\iota: \overline{\mathbb{Q}}_{l} \cong \mathbb{C}$, there exists $v \mid l$ such that the character $\alpha_{2} / \alpha_{1}$ has order strictly greater than 5 .

This character is given by the formula

$$
\alpha_{2} / \alpha_{1}=\prod_{\tau \in J_{1}} \omega_{\tau}^{\lambda_{\iota}-1_{\tau, 1}+1-\lambda_{\iota}-1_{\tau, 2}} \prod_{\tau \in J_{2}} \omega_{\tau}^{-\left(\lambda_{\iota}-1_{\tau, 1}+1-\lambda_{\iota}-1_{\tau, 2}\right)}
$$

Let $f_{v}$ denote the absolute residue degree of $E_{v}$, and fix an embedding $\tau_{0}: E_{v} \hookrightarrow \overline{\mathbb{Q}}_{l}$. Writing $\varphi \in \operatorname{Gal}\left(E_{v} / \mathbb{Q}_{l}\right)$ for the arithmetic Frobenius, this allows us to identify $S_{v}=\left\{\tau_{0}, \varphi \circ \tau_{0}, \ldots, \varphi^{f_{v}-1} \circ \tau_{0}\right\}=\left\{0,1, \ldots, f_{v}-1\right\}$, so that the order of the character $\alpha_{2} / \alpha_{1}$ is equal to the order of the image of the integer

$$
\sum_{i \in J_{1}} l^{i}\left(\lambda_{\iota^{-1} \tau, 1}+1-\lambda_{\iota^{-1} \tau, 2}\right)-\sum_{i \in J_{2}} l^{i}\left(\lambda_{\iota^{-1} \tau, 1}+1-\lambda_{\iota^{-1} \tau, 2}\right)
$$


in the group $\mathbb{Z} /\left(l^{f_{v}}-1\right) \mathbb{Z}$. This integer is not zero (since it is not even zero modulo $l$ ), so to prove the lemma it is enough to show that for $l$ sufficiently large, we have

$$
5\left|\sum_{i \in J_{1}} l^{i}\left(\lambda_{\iota^{-1} \tau, 1}+1-\lambda_{\iota^{-1} \tau, 2}\right)-\sum_{i \in J_{2}} l^{i}\left(\lambda_{\iota^{-1} \tau, 1}+1-\lambda_{\iota^{-1} \tau, 2}\right)\right|<l^{f_{v}}-1 .
$$

However, the left hand side here is less than or equal to

$$
5 \sum_{i=0}^{f_{v}-1} l^{i}\left(\lambda_{\iota^{-1} \tau, 1}+1-\lambda_{\iota^{-1} \tau, 2}\right) \leq 5 k\left(l^{f_{v}}-1\right) /(l-1),
$$

so it is enough to take $l>5 k+1$.

Proposition 7.6. Let $r \geq 1$ be an integer. Let $E / F$ be an imaginary $C M$ quadratic extension of a totally real field, and suppose that for every RAESDC automorphic representation $(\pi, \chi)$ of $\mathrm{GL}_{2}\left(\mathbb{A}_{F}\right)$ such that $\pi$ is not automorphically induced from a CM extension, the $r^{\text {th }}$ symmetric power $\operatorname{Sym}^{r} r_{\iota}(\pi)$ is automorphic.

Then for every $R A C S D C$ automorphic representation $\Pi$ of $\mathrm{GL}_{2}\left(\mathbb{A}_{E}\right)$ which is not automorphically induced from a quadratic extension, the $r^{\text {th }}$ symmetric power $\operatorname{Sym}^{r} r_{\iota}(\Pi)$ is automorphic.

Proof. By Lemma 7.5 we can find a prime $l>2(r+2)$ and an isomorphism $\iota: \overline{\mathbb{Q}}_{l} \cong \mathbb{C}$ such that the residual representation $\overline{r_{\iota}(\Pi)}$ is irreducible and its image contains a conjugate of $\mathrm{SL}_{2}\left(\mathbb{F}_{l}\right)$. We can further assume that $E$ and $\pi$ are unramified above $l$, and that for each embedding $\tau: E \hookrightarrow \overline{\mathbb{Q}}_{l}$, the elements of the set $\mathrm{HT}_{\tau}\left(\operatorname{Sym}^{r} r_{\iota}(\Pi)\right)$ lie in an interval of length at most $l-2$, and that $l$ is split in $E$.

There exists a continuous character $\varphi: G_{E} \rightarrow \overline{\mathbb{F}}_{l}^{\times}$such that $\left(\overline{r_{\iota}(\Pi)} \otimes \varphi\right)^{c} \cong \overline{r_{\iota}(\Pi)} \otimes \varphi$. In particular, the representation $\left(\overline{r_{\iota}(\Pi)} \otimes \varphi\right)$ extends to a homomorphism $\bar{R}: G_{F} \rightarrow \mathrm{GL}_{2}\left(\overline{\mathbb{F}_{l}}\right)$, which is odd (cf. the proof of [Tho15, Theorem 8.1]). Applying [BLGGT14, Theorem 4.4.1] and a standard extension of this result to a totally real base field, we can construct a lift $R: G_{F} \rightarrow \mathrm{GL}_{2}\left(\overline{\mathbb{Z}}_{l}\right)$ which is potentially diagonalizable in the sense of [BLGGT14, §1.4], and unramified almost everywhere. The representations $\left.R\right|_{G_{E}}$ and $r_{\iota}(\Pi)$ are potentially diagonalizable by [BLGGT14, Lemma 1.4.3], and it now follows from [BLGGT14, Theorem 4.2.1] and soluble descent that $R$ itself is automorphic.

By assumption, the representation $\operatorname{Sym}^{r} R$ is automorphic, hence $\operatorname{Sym}^{r} \overline{r_{\iota}(\Pi)}$ is (in the terminology of [BLGGT14]) residually potentially diagonalizably automorphic. Applying [BLGGT14, Theorem 4.2.1] once more, we deduce that $\operatorname{Sym}^{r} r_{\iota}(\Pi)$ is automorphic.

Proof of Theorem \%.1. Let $F$ be a totally real field. Let $\pi$ be a cuspidal automorphic representation of $\mathrm{GL}_{2}\left(\mathbb{A}_{F}\right)$ such that $\pi_{\infty}$ is essentially square integrable and $\mathrm{Sym}^{2} \pi$ is cuspidal. We must show that the $r^{\text {th }}$ symmetric power lifting of $\pi$ exists.

Choose an imaginary $\mathrm{CM}$ quadratic extension $E / F$. By Lemma 7.4, there exists a Hecke character $\chi: E^{\times} \backslash \mathbb{A}_{E}^{\times} \rightarrow \mathbb{C}^{\times}$such that $\pi_{E} \otimes \chi$ is RACSDC. Combining our hypothesis with Proposition 7.6, we deduce that the $r^{\text {th }}$ symmetric power lifting of $\pi_{E} \otimes \chi$ exists, as a cuspidal automorphic representation of $\mathrm{GL}_{r+1}\left(\mathbb{A}_{E}\right)$. The representation $\mathrm{Sym}^{r} \pi_{E}$ thus exists and descends to a cuspidal automorphic representation $\Pi$ of $\mathrm{GL}_{r+1}\left(\mathbb{A}_{F}\right)$.

It remains to show that for each place $v \nmid \infty$ of $F, \Pi_{v}$ has the correct form. Let $v$ be a finite place of $F$, and let $E_{0}$ be a quadratic imaginary $\mathrm{CM}$ extension of $F$ in which $v$ splits. Applying Lemma 7.4 once more, we can find a Hecke character $\chi_{0}: E_{0}^{\times} \backslash \mathbb{A}_{E_{0}}^{\times} \rightarrow \mathbb{C}^{\times}$such that $\pi_{E_{0}} \otimes \chi_{0}$ is RACSDC. Applying local-global compatibility for $\operatorname{Sym}^{r}\left(\pi_{E_{0}} \otimes \chi_{0}\right)$, a twist of $\Pi_{E_{0}}$, we deduce that

$$
\operatorname{Sym}^{r} \operatorname{rec}_{F_{v}} \pi_{v}=\operatorname{rec}_{F_{v}} \Pi_{v} .
$$

Since $v$ was arbitrary, this completes the proof. 


\section{References}

[AC89] James Arthur and Laurent Clozel. Simple algebras, base change, and the advanced theory of the trace formula, volume 120 of Annals of Mathematics Studies. Princeton University Press, Princeton, NJ, 1989.

[Art] James Arthur. The endoscopic classification of representations: orthogonal and symplectic groups. To appear as a Colloquium Publication of the American Mathematical Society.

[Art03] James Arthur. A stable trace formula. III. Proof of the main theorems. Ann. of Math. (2), 158(3):769-873, 2003.

[Art05] James Arthur. An introduction to the trace formula. In Harmonic analysis, the trace formula, and Shimura varieties, volume 4 of Clay Math. Proc., pages 1-263. Amer. Math. Soc., Providence, RI, 2005.

[BH06] Colin J. Bushnell and Guy Henniart. The local Langlands conjecture for GL(2), volume 335 of Grundlehren der Mathematischen Wissenschaften [Fundamental Principles of Mathematical Sciences]. Springer-Verlag, Berlin, 2006.

[BLGG11] Thomas Barnet-Lamb, Toby Gee, and David Geraghty. The Sato-Tate conjecture for Hilbert modular forms. J. Amer. Math. Soc., 24(2):411-469, 2011.

[BLGGT14] Thomas Barnet-Lamb, Toby Gee, David Geraghty, and Richard Taylor. Potential automorphy and change of weight. Ann. of Math. (2), 179(2):501-609, 2014.

[BLGHT11] Tom Barnet-Lamb, David Geraghty, Michael Harris, and Richard Taylor. A family of CalabiYau varieties and potential automorphy II. Publ. Res. Inst. Math. Sci., 47(1):29-98, 2011.

[BR93] Don Blasius and Jonathan D. Rogawski. Motives for Hilbert modular forms. Invent. Math., 114(1):55-87, 1993.

[Cas80] W. Casselman. The unramified principal series of $\mathfrak{p}$-adic groups. I. The spherical function. Compositio Math., 40(3):387-406, 1980.

[CG10] Neil Chriss and Victor Ginzburg. Representation theory and complex geometry. Modern Birkhäuser Classics. Birkhäuser Boston, Inc., Boston, MA, 2010. Reprint of the 1997 edition.

[CHL11] Laurent Clozel, Michael Harris, and Jean-Pierre Labesse. Endoscopic transfer. In On the stabilization of the trace formula, volume 1 of Stab. Trace Formula Shimura Var. Arith. Appl., pages 475-496. Int. Press, Somerville, MA, 2011.

[CHT08] Laurent Clozel, Michael Harris, and Richard Taylor. Automorphy for some l-adic lifts of automorphic mod $l$ Galois representations. Publ. Math. Inst. Hautes Études Sci., (108):1-181, 2008. With Appendix A, summarizing unpublished work of Russ Mann, and Appendix B by Marie-France Vignéras.

[Clo86] Laurent Clozel. On limit multiplicities of discrete series representations in spaces of automorphic forms. Invent. Math., 83(2):265-284, 1986.

[Clo93] Laurent Clozel. On the cohomology of Kottwitz's arithmetic varieties. Duke Math. J., 72(3):757795, 1993.

[Clo11] Laurent Clozel. Identités de caractères en la place archimédienne. In On the stabilization of the trace formula, volume 1 of Stab. Trace Formula Shimura Var. Arith. Appl., pages 351-367. Int. Press, Somerville, MA, 2011.

[CT14] Laurent Clozel and Jack A. Thorne. Level-raising and symmetric power functoriality, I. Compos. Math., 150(5):729-748, 2014. 
[CT15] Laurent Clozel and Jack A. Thorne. Level raising and symmetric power functoriality, II. Ann. of Math. (2), 181(1):303-359, 2015.

[DCLP88] C. De Concini, G. Lusztig, and C. Procesi. Homology of the zero-set of a nilpotent vector field on a flag manifold. J. Amer. Math. Soc., 1(1):15-34, 1988.

[FL82] Jean-Marc Fontaine and Guy Laffaille. Construction de représentations p-adiques. Ann. Sci. École Norm. Sup. (4), 15(4):547-608 (1983), 1982.

[Gee11] Toby Gee. Automorphic lifts of prescribed types. Math. Ann., 350(1):107-144, 2011.

[Ger] David Geraghty. Modularity lifting theorems for ordinary Galois representations. Preprint.

[GGP12] Wee Teck Gan, Benedict H. Gross, and Dipendra Prasad. Symplectic local root numbers, central critical $L$ values, and restriction problems in the representation theory of classical groups. Astérisque, (346):1-109, 2012. Sur les conjectures de Gross et Prasad. I.

[GJ78] Stephen Gelbart and Hervé Jacquet. A relation between automorphic representations of GL(2) and GL(3). Ann. Sci. École Norm. Sup. (4), 11(4):471-542, 1978.

[Gue11] Lucio Guerberoff. Modularity lifting theorems for Galois representations of unitary type. Compos. Math., 147(4):1022-1058, 2011.

[Har09] Michael Harris. Potential automorphy of odd-dimensional symmetric powers of elliptic curves and applications. In Algebra, arithmetic, and geometry: in honor of Yu. I. Manin. Vol. II, volume 270 of Progr. Math., pages 1-21. Birkhäuser Boston Inc., Boston, MA, 2009.

[HSBT10] Michael Harris, Nick Shepherd-Barron, and Richard Taylor. A family of Calabi-Yau varieties and potential automorphy. Ann. of Math. (2), 171(2):779-813, 2010.

[JS81a] H. Jacquet and J. A. Shalika. On Euler products and the classification of automorphic forms. II. Amer. J. Math., 103(4):777-815, 1981.

[JS81b] H. Jacquet and J. A. Shalika. On Euler products and the classification of automorphic representations. I. Amer. J. Math., 103(3):499-558, 1981.

[Kim03] Henry H. Kim. Functoriality for the exterior square of $\mathrm{GL}_{4}$ and the symmetric fourth of $\mathrm{GL}_{2}$. J. Amer. Math. Soc., 16(1):139-183, 2003. With appendix 1 by Dinakar Ramakrishnan and appendix 2 by Kim and Peter Sarnak.

[KL87] David Kazhdan and George Lusztig. Proof of the Deligne-Langlands conjecture for Hecke algebras. Invent. Math., 87(1):153-215, 1987.

[KS02] Henry H. Kim and Freydoon Shahidi. Functorial products for $\mathrm{GL}_{2} \times \mathrm{GL}_{3}$ and the symmetric cube for $\mathrm{GL}_{2}$. Ann. of Math. (2), 155(3):837-893, 2002. With an appendix by Colin J. Bushnell and Guy Henniart.

[Lab11] J.-P. Labesse. Changement de base CM et séries discrètes. In On the stabilization of the trace formula, volume 1 of Stab. Trace Formula Shimura Var. Arith. Appl., pages 429-470. Int. Press, Somerville, MA, 2011.

[Lan70] R. P. Langlands. Problems in the theory of automorphic forms. In Lectures in modern analysis and applications, III, pages 18-61. Lecture Notes in Math., Vol. 170. Springer, Berlin, 1970.

[Lan89] R. P. Langlands. On the classification of irreducible representations of real algebraic groups. In Representation theory and harmonic analysis on semisimple Lie groups, volume 31 of Math. Surveys Monogr., pages 101-170. Amer. Math. Soc., Providence, RI, 1989. 
[LS87] R. P. Langlands and D. Shelstad. On the definition of transfer factors. Math. Ann., 278(14):219-271, 1987.

[Lus89] George Lusztig. Affine Hecke algebras and their graded version. J. Amer. Math. Soc., 2(3):599635, 1989.

[Mat64] Hideya Matsumoto. Générateurs et relations des groupes de Weyl généralisés. C. R. Acad. Sci. Paris, 258:3419-3422, 1964.

[Mat89] Hideyuki Matsumura. Commutative ring theory, volume 8 of Cambridge Studies in Advanced Mathematics. Cambridge University Press, Cambridge, second edition, 1989. Translated from the Japanese by M. Reid.

[Mín11] Alberto Mínguez. Unramified representations of unitary groups. In On the stabilization of the trace formula, volume 1 of Stab. Trace Formula Shimura Var. Arith. Appl., pages 389-410. Int. Press, Somerville, MA, 2011.

[Mœg07] Colette Mœglin. Classification et changement de base pour les séries discrètes des groupes unitaires p-adiques. Pacific J. Math., 233(1):159-204, 2007.

[Mok15] Chung Pang Mok. Endoscopic classification of representations of quasi-split unitary groups. Mem. Amer. Math. Soc., 235(1108):vi+248, 2015.

[MW] Colette Møglin and Jean-Loup Waldspurger. Stabilisation de la formule des trace tordue I X. Preprint. Available at http://webusers.imj-prg.fr/ jean-loup.waldspurger/.

[Pro98] Kerrigan Procter. Parabolic induction via Hecke algebras and the Zelevinsky duality conjecture. Proc. London Math. Soc. (3), 77(1):79-116, 1998.

[Ram00] Dinakar Ramakrishnan. Modularity of the Rankin-Selberg $L$-series, and multiplicity one for SL(2). Ann. of Math. (2), 152(1):45-111, 2000.

[Ree94] Mark Reeder. On the Iwahori-spherical discrete series for $p$-adic Chevalley groups; formal degrees and L-packets. Ann. Sci. École Norm. Sup. (4), 27(4):463-491, 1994.

[Ree00] Mark Reeder. Matrices for affine Hecke modules. J. Algebra, 231(2):758-785, 2000.

[She] D. Shelstad. On spectral transfer factors in real twisted endoscopy. Preprint.

[She82] D. Shelstad. L-indistinguishability for real groups. Math. Ann., 259(3):385-430, 1982.

[She10] D. Shelstad. Tempered endoscopy for real groups. II. Spectral transfer factors. In Automorphic forms and the Langlands program, volume 9 of Adv. Lect. Math. (ALM), pages 236-276. Int. Press, Somerville, MA, 2010.

[Tay89] Richard Taylor. On Galois representations associated to Hilbert modular forms. Invent. Math., 98(2):265-280, 1989.

[Tay08] Richard Taylor. Automorphy for some $l$-adic lifts of automorphic mod $l$ Galois representations. II. Publ. Math. Inst. Hautes Études Sci., (108):183-239, 2008.

[Tho12] Jack A. Thorne. On the automorphy of $l$-adic Galois representations with small residual image. Journal of the Inst. of Math. Jussieu, 11(4):855-920, 2012. With an appendix by Robert Guralnick, Florian Herzig, Richard Taylor and Jack Thorne.

[Tho14] Jack A. Thorne. Raising the level for $\mathrm{GL}_{n}$. Forum Math. Sigma, 2:e16, 35, 2014.

[Tho15] Jack A. Thorne. Automorphy lifting for residually reducible $l$-adic Galois representations. J. Amer. Math. Soc., 28(3):785-870, 2015. 
[Tit79] J. Tits. Reductive groups over local fields. In Automorphic forms, representations and Lfunctions (Proc. Sympos. Pure Math., Oregon State Univ., Corvallis, Ore., 1977), Part 1, Proc. Sympos. Pure Math., XXXIII, pages 29-69. Amer. Math. Soc., Providence, R.I., 1979.

[TY07] Richard Taylor and Teruyoshi Yoshida. Compatibility of local and global Langlands correspondences. J. Amer. Math. Soc., 20(2):467-493, 2007.

[Wal10] J.-L. Waldspurger. Les facteurs de transfert pour les groupes classiques: un formulaire. Manuscripta Math., 133(1-2):41-82, 2010. 